\title{
Contribución al conocimiento de la flora vascular de la Reserva Biológica Huilo Huilo, Región de Los Ríos, Chile
}

\author{
A contribution to the knowledge of the flora of Huilo Huilo Biological Reserve, Región \\ de Los Ríos, Chile
}

\author{
Sebastián Teillier ${ }^{1 *}$, Jorge Macaya-Berti², Catherinne Bonnemaison ${ }^{3}$, Javiera Delaunoy $^{3} \&$ Alicia \\ MARTICORENA ${ }^{4}$
}

\author{
${ }^{1}$ Escuela de Arquitectura del Paisaje, Universidad Central de Santiago de Chile. Santa Isabel 1186. Santiago. \\ ${ }^{2}$ Investigador independiente: Los Nogales 823, Providencia, Santiago \\ ${ }^{3}$ Fundación Huilo Huilo. Oficina Central, Av.Vitacura 2909, of. 1112 Las Condes, Santiago. \\ ${ }^{4}$ Departamento de Botánica, Facultad de Ciencias Naturales y Oceanográficas, Universidad de Concepción, Casilla 160-C, \\ Concepción, Chile. \\ *steillier@gmail.com
}

\section{RESUMEN}

Las áreas protegidas estatales juegan un papel decisivo en la conservación de la naturaleza; marco en que las reservas privadas son aliadas importantes, pues mejoran su representatividad y aumentan su superficie. La Reserva Biológica Huilo Huilo se encuentra en la ecorregión de los bosques templados del sur de Sudamérica e incluye diversos tipos de bosques de Nothofagus y vegetación andina. Se ubica en la Región de Los Ríos, localidad de Neltume (3951'S-7157’W) entre 500 y $2.000 \mathrm{~m}$ altitud. Los resultados corresponden al estudio de un cuadrante de $c a .11$ x $20 \mathrm{~km}$. La riqueza de la flora vascular alcanza a 406 especies, que se distribuyen en 95 familias y 230 géneros. Las familias con más especies son: Asteraceae, con 60 (14,8\% del total); Poaceae, con 43 (10,6\%); Cyperaceae, con 21 (5,1\%) e Hymenophyllaceae con 16 (3,9\%). Entre los géneros destacan por riqueza, Hymenophyllum con 13 especies; Carex y Senecio con 9; Agrostis, Baccharis y Ranunculus con 7. La mayor parte de las especies son nativas: $340(83,7 \%)$; las alóctonas asilvestradas alcanzan a 66 (16,3\%). Entre las nativas, predominan las plantas endémicas de Chile y Argentina, con 266 especies $(78,2 \%$ del total de nativas); seguidas por las nativas, no endémicas ni de Chile ni de Argentina, con 54 (15,9\%) y las endémicas de Chile, con 20 (5,9\%). Entre las endémicas de Chile, Elaphoglossum fonkii (Lomariopsidae, Pteridophyta) es exclusiva de las Regiones de Los Ríos y de Los Lagos, y Ercilla syncarpellata (Phytolaccaceae) se ha recolectado entre las Regiones de La Araucanía y de Los Lagos. Primula comberi (Primulaceae) es nueva para Chile, lo mismo que Oenothera stricta var. altissima (Onagraceae). Se amplía la distribución de Trichomanes exsectum (Hymenophyllaceae) que no era conocido para la cordillera de los Andes, de Lycopodium alboffii (Lycopodiaceae), nuevo límite norte de distribución, de Epilobium densifolium (Onagraceae), también con un nuevo límite sur y la de Ranunculus hydrophilus (Ranunculaceae), primera mención para la Región de Los Ríos. Entre las formas de vida predominan los hemicriptófitos con un $60 \%$, seguidos por los fanerófitos, con $11,3 \%$ y los nanofanerófitos, con $11 \%$. Se encontraron 18 especies que han sido incluidas en alguna categoría de amenaza, las más destacadas Greigia pearcei (Bromeliaceae), Elaphoglossum fonkii (Lomariopsidaceae), Fascicularia bicolor (Bromeliaceae), Libertia tricocca (Iridaceae) y Trichomanes exsectum (Hymenophyllaceae). Un $82,3 \%$ de las familias y un $77 \%$ de los géneros de los bosques australes templados de Sudamérica están representados en la reserva. Al nivel de riqueza por comunidad, el bosque valdiviano es el más rico con 136 de las 337 especies nativas del área de estudio; lo siguen los de Nothofagus dombeyi (coigüe), con 96; el de Nothofagus antarctica (ñirre), con 45 y el de Nothofagus pumilio (lenga) con 34. Dado que la riqueza está relacionada con la superficie, es notable que el bosque de tipo valdiviano, que es uno de los que ocupa la menor superficie, tenga la riqueza más alta.

Palabras clave: Flora de Chile, flora de la Región de Los Ríos, Reserva Biológica Huilo Huilo, Primula comberi.

\begin{abstract}
Although state Reserves play a major role in biodiversity conservation, private Reserves may play a complementary role by increasing surface and ecological representation. The Reserva Biologica Huilo Huilo occupies an area of 100000 ha and is situated in the temperate austral forest ecoregion of South America and includes Nothofagus forests and various types of high Andean vegetation. The Reserve is located at Región de Los Ríos, near the locality of Neltume (39 $51^{\prime}$ 'S $71^{\circ} 57^{\prime} \mathrm{W}$ ) at $500-2000 \mathrm{~m}$ altitude. The results reported were obtained within an area of 11 x $20 \mathrm{~km}$. Specific richness was 406 of
\end{abstract}


Flora vascular de la Reserva Biológica Huilo Huilo: TeILLIER, S. ET AL.

vascular plant species belonging to 95 families and 230 genera. The richest families were asteraceae (60 species, $14.8 \%)$, poaceae (43 species, 10.6\%), cyperaceae (21 species, 5.1\%) and hymenophyllaceae (16 species, 3.9\%). the richest genera were Hymenophyllum (13 species), Carex and Senecio ( 9 species each). Alien species constituted 16.3\% of the total and native species $83.7 \%$, of which $79 \%$ are endemic to Chile and Argentina and $6 \%$ only to Chile. The Chilean endemics with most restricted distributions are Elaphoglossum fonkii (Lomariopsidae, Pteridophyta) exclusive of Regiones de Los Ríos and de Los Lagos, and Ercilla syncarpellata (Phytolaccaceae) from Región de La Araucanía, de Los Ríos and de Los Lagos. Primula comberi (Primulaceae) and Oenothera stricta var. altissima (Onagraceae) are reported as a new species for Chile. We report for the first time Trichomanes exsectum at the cordillera de los Andes; Ranunculus hydrophilus is mentioned for the first time at Region de Los Ríos; finally distribution areas were enlarged for Epilobium densifolium (a new southern limit in Chile) and Lycopodium alboffii (a new northern limit in Chile). Hemikryptophyta is the richest taxon with Raunkiaer's life form (60\%), followed by macrophanerophyta (11.3\%) and nanopanerophyta (11\%). Eighteen species are classified as threatened, most notably Greigia pearcei (Bromeliaceae), Elaphoglossum fonkii (Lomariopsidaceae), Fascicularia bicolor (Bromeliaceae), Libertia tricocca (Iridaceae) and Trichomanes exsectum (Hymenophyllaceae). About $82.3 \%$ of plant families and $77 \%$ of the genera of the austral temperate South American forest are represented at Reserva Biológica Huilo Huilo. Genera of the Pteridophyta and Magnoliopsida s.l. are the best represented with $82.6 \%$ and $76 \%$, respectively. The Valdivian type forest is the richest community (136 species), followed by Nothofagus dombeyi forest ( 96 species), Nothofagus antarctica forest (45 species) and Nothofagus pumilio forest (34 species). The Valdivian type forest, although occupying a relatively small area is that with the highest species richness.

Keywords: Flora of Chile, Flora of Los Rios Region, Huilo Huilo Biological reserve, Primula comberi.

\section{INTRODUCCIÓN}

Entre los objetivos específicos de la política nacional de áreas silvestres protegidas del Estado de Chile está el de "fomentar la incorporación del sector privado en la creación y gestión de áreas protegidas" (CONAMA 2005). En el país existe hoy día una red de reservas privadas destinadas a la conservación de la naturaleza que complementa los esfuerzos que el Estado realiza en esa misma dirección materializando lo que se denomina "el sentido de custodia de la naturaleza". En la Región de Los Ríos (XIV) las unidades del sistema nacional de áreas protegidas del Estado (SNASPE) alcanzan a unas $20.000 \mathrm{ha}$, sin considerar las áreas birregionales como el Parque Nacional Villarrica y el Parque Nacional Puyehue; a las que se suman, en términos de los esfuerzos de la conservación de la naturaleza, unas 198.546 ha de áreas de privados (A. Farías, com. pers.).

Una de las mayores áreas privadas destinadas a la conservación de la naturaleza de la región es la Reserva Biológica Huilo Huilo. Ésta, con una superficie actual de cerca de 100.000 ha, desde 1996 se encuentra bajo un modo de manejo que va desde la creación de zonas intangibles, donde se intenta reintroducir al huemul (Hippocamelus bisulcus), hasta zonas donde se llevan a cabo actividades de turismo y una explotación forestal selectiva y de baja intensidad. El objetivo de la reserva es contribuir a la conservación y a la recuperación del bosque lluvioso templado, integrando a las comunidades aledañas con el fin de mejorar su calidad de vida para las presentes y futuras generaciones (Fundación Huilo Huilo 2009).

La reserva alberga ecosistemas que han sido incluidos en la ecorregión del Bosque Templado Valdiviano (Dinerstein et al. 1995). La singularidad de estos bosques ha sido puesta en relieve por Armesto et al. (1996), quienes han demostrado su carácter de isla biogeográfica; lo que se ve reflejado en un grado de endemismo que alcanza al $90 \%$ de la flora vascular de la ecorregión (Smith 2001). Estos bosques australes sólo se encuentran en el sur de Chile y Argentina y ocupan apenas una franja de $2.000 \mathrm{~km}$ de largo por unos $120 \mathrm{~km}$ de ancho (Armesto et al. 1996). De acuerdo con Myers et al. (2000), estos bosques templados de Chile son considerados ecosistemas clave para la conservación de la biodiversidad a nivel mundial (hotspot).

Si bien el bosque valdiviano sensu stricto (Schmithüsen 1956, Luebert \& Pliscoff 2005) comprende sólo a los bosques laurifolios, dominados por Eucryphia cordifolia, Nothofagus dombeyi, Weinmannia trichosperma, Laureliopsis philippiana, Aextoxicon punctatum y Luma apiculata; aquí hemos seguido la propuesta de Dinerstein (1995), que incluye otros tipos de bosque, particularmente el caducifolio andino de lenga (Nothofagus pumilio). De acuerdo con lo expresado, los bosques templados australes de la ecozona "valdiviana" se extienden, en nuestro país, entre los $35^{\circ}$ y $55^{\circ} \mathrm{S}$ y se encuentran también en el sur de Argentina. La riqueza de flora vascular que albergan sus comunidades se ha estimado en unos 200 géneros y 700 800 especies (Arroyo et al. 1995).

Respecto de estos bosques se afirma que casi todos presentan actualmente algún tipo de intervención antrópica y que su área de distribución original se ha reducido en al menos un tercio desde comienzos de la colonización (Claude 1997) o incluso en un $40 \%$ (WWF 2009). La organización Word Wildlife Foundation sostiene que se trata de una ecozona (ecorregión) que se encontraría significativamente amenazada, sindicando como factores principales la habilitación de tierras para la agricultura y la fruticultura, el cultivo de praderas con plantas exóticas para el pastoreo y la destrucción y sustitución del bosque nativo 
por árboles alóctonos de más rápido crecimiento como Pinus contorta, Pinus radiata, Pseudotsuga menziesii, Sequoia sempervirens y Eucalyptus spp.

El Estado de Chile desde principios del siglo XX ha mostrado preocupación por la conservación de estos bosques estableciendo áreas protegidas, entre las que destacan el Parque Nacional Puyehue y el Parque Nacional Alerce Andino. En 1994 se crea en el nororiente de la Región de Los Ríos, la Reserva Nacional Mocho-Choshuenco, que limita hacia el oriente con la que la Reserva Biológica Huilo Huilo, desempeñando ambas un rol complementario en la protección de la naturaleza del sector.

Existen varias publicaciones referidas a la flora vascular de esta ecorregión entre las que citamos la del Parque Nacional Tolhuaca (Ramírez 1978), la del Parque Nacional Puyehue (Muñoz-Schick 1980), la del Parque Nacional Laguna San Rafael (Teillier \& Marticorena 2002), la de la Monumento Natural Contulmo (Baeza et al. 1999), la de la Reserva Forestal Malalcahuello (Becerra \& Faúndez 1999) y la de la Reserva Nacional Tamango (Tomé et al. 2007), sin embargo, para el área de estudio no existen aún publicaciones.

Desde el punto de vista de la conservación de la flora y vegetación, el área de la reserva Huilo Huilo, y en general, la cuenca del lago Pirehueico, han sido incluidas en varias oportunidades entre los sitios prioritarios para la conservación de la biodiversidad (Benoit 1989, Muñoz et al. 1996, CONAMA 2005, Farías et al. 2004).
El objetivo de este trabajo es contribuir al conocimiento de la flora vascular de la Reserva Biológica Huilo Huilo con el fin de dar a conocer y valorar su patrimonio natural y contribuir al conocimiento general de la flora de la ecorregión y del país.

\section{MATERIALES Y MÉTODOS}

\section{UBICACIÓN}

La Reserva Biológica Huilo Huilo, se ubica en la comuna de Panguipulli, localidad de Neltume (39 $51^{\prime} \mathrm{S}-71^{\circ} 57^{\prime} \mathrm{W}$ ), entre los 400 y los $1.800 \mathrm{~m}$ de altitud. La reserva comprende más de 100.000 ha; de las que unas 20.000 ha son el objetivo de este trabajo. Se exploró el sector ubicado entre la ribera poniente del lago Pirehueico (39 56'09' S- $71^{\circ} 48^{\prime} 31^{\prime \prime} \mathrm{W}$ ), las estribaciones occidentales de la cordillera de Arquilhue-cerro Piedra del Encanto (39 $59^{\circ}$ '43' S-71 $48^{\circ}$ 02"W), la ladera oriente del volcán Mocho-Choshuenco $\left(39^{\circ} 55^{\prime} 15^{\prime \prime} \mathrm{S}-71^{\circ} 59^{\prime} 20^{\prime \prime} \mathrm{W}\right)$, el río Fuy (39॰51'10" S-71 $\left.57^{\circ} 30^{\prime \prime} \mathrm{W}\right)$ y el área norte del valle del río Pillanleufú $\left(39^{\circ} 59^{\prime} 43^{\prime \prime} \mathrm{S}-71^{\circ} 48^{\prime} 02^{\prime \prime} \mathrm{W}\right)$. Existe en el área un gradiente de altitud, que tiene su parte más baja en el río Fuy ( 400 m s.n.m.) ubicado al norte de ella y máximos en el volcán Mocho-Choshuenco, donde el límite de la vegetación se ubica por los $1.600 \mathrm{~m}$ de altitud y en las estribaciones occidentales de la cordillera de Arquilhue, donde se accedió hasta los 1.400 m s.n.m (Fig. 1).

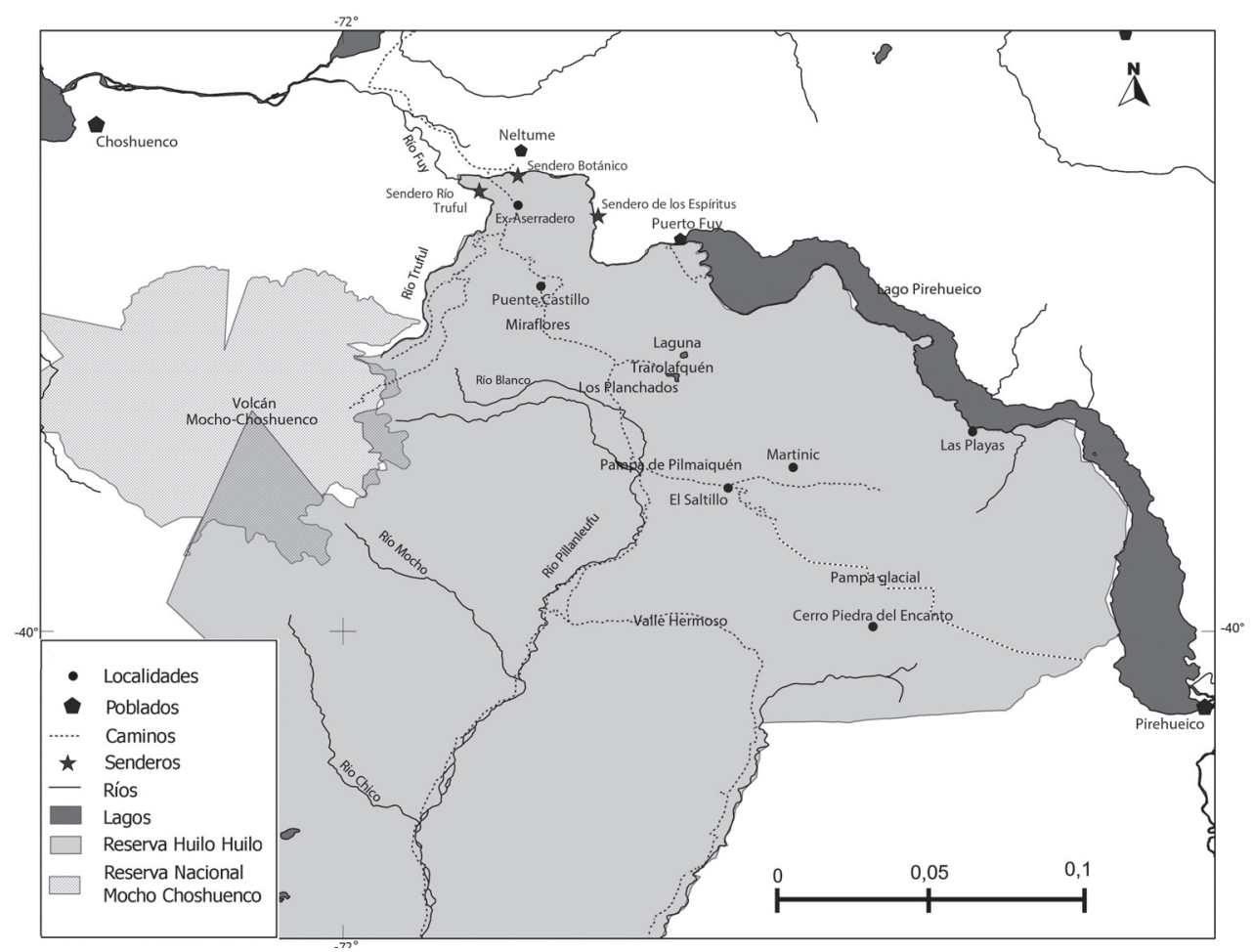

Figura 1. Flora vascular de la Reserva Biológica de Huilo Huilo (Región de Los Ríos, Chile): área de estudio.

Figure 1. Vascular flora at Huilo Huilo Reserve (Región de Los Ríos, Chile): study site. 
CLIMA

De acuerdo con Di Castri \& Hayek (1976), el área de estudio se encuentra bajo un régimen climático de tendencia oceánica, siendo de éste carácter templado-húmedo, con registro de precipitaciones durante todo el año y ausencia de déficit hídrico. Las precipitaciones anuales alcanzan a unos $2.000 \mathrm{~mm}$, las que se registran tanto en forma de lluvia como de nieve; ésta última es más frecuente en invierno, sobre los $600 \mathrm{~m}$ de altitud.

\section{VEGETACIÓN}

De acuerdo con los datos de la literatura y con lo observado en terreno la vegetación del área de Huilo Huilo se clasificó, con fines de organizar un muestreo estratificado, en los siguientes tipos de comunidades:

BosQue DE TIPO VALDIVIANO: los árboles dominantes son los siempreverdes Eucryphia cordifolia, Aextoxicon punctatum, Persea lingue, Weinmannia trichosperma y Myrceugenia planipes; se caracteriza, además, por una riqueza importante de helechos (Fig. 2). En la reserva tiene una distribución algo restringida y se ubica en los sectores más bajos, entre
400 y 550 m s.n.m.; principalmente en torno a los ríos Truful y Fuy. Por su composición de especies y dominancia, correspondería a la asociación de Eucryphia cordifolia y Weinmannia trichosperma de la formación del bosque laurifolio de Los Lagos (Gajardo 1994). Desde el punto de vista fitosociológico esta comunidad se integra en la asociación Dombeyo-Eucryphietum Oberdorfer.

Bosque DE NothofaGuS DOMBEY: bosque siempreverde o mixto (Fig. 3); en las partes más bajas el coigüe se asocia con Saxegothaea conspicua y Laureliopsis philippiana; en tanto que en las partes ubicadas a mayor altitud aparece Nothofagus alpina, una especie caducifolia. Es el tipo de vegetación que tiene la mayor distribución en la reserva, $\mathrm{y}$ se encuentra desde los 500 hasta cerca de $1.000 \mathrm{~m}$ de altitud. Por la composición de su flora estos bosques podrían referirse a la asociación de Nothofagus dombeyi-Laurelia philippiana, de la formación del bosque laurifolio de Los Lagos; y a la de Nothofagus alpina-Nothofagus dombeyi, de la formación del bosque caducifolio mixto de la cordillera andina (Gajardo 1994).

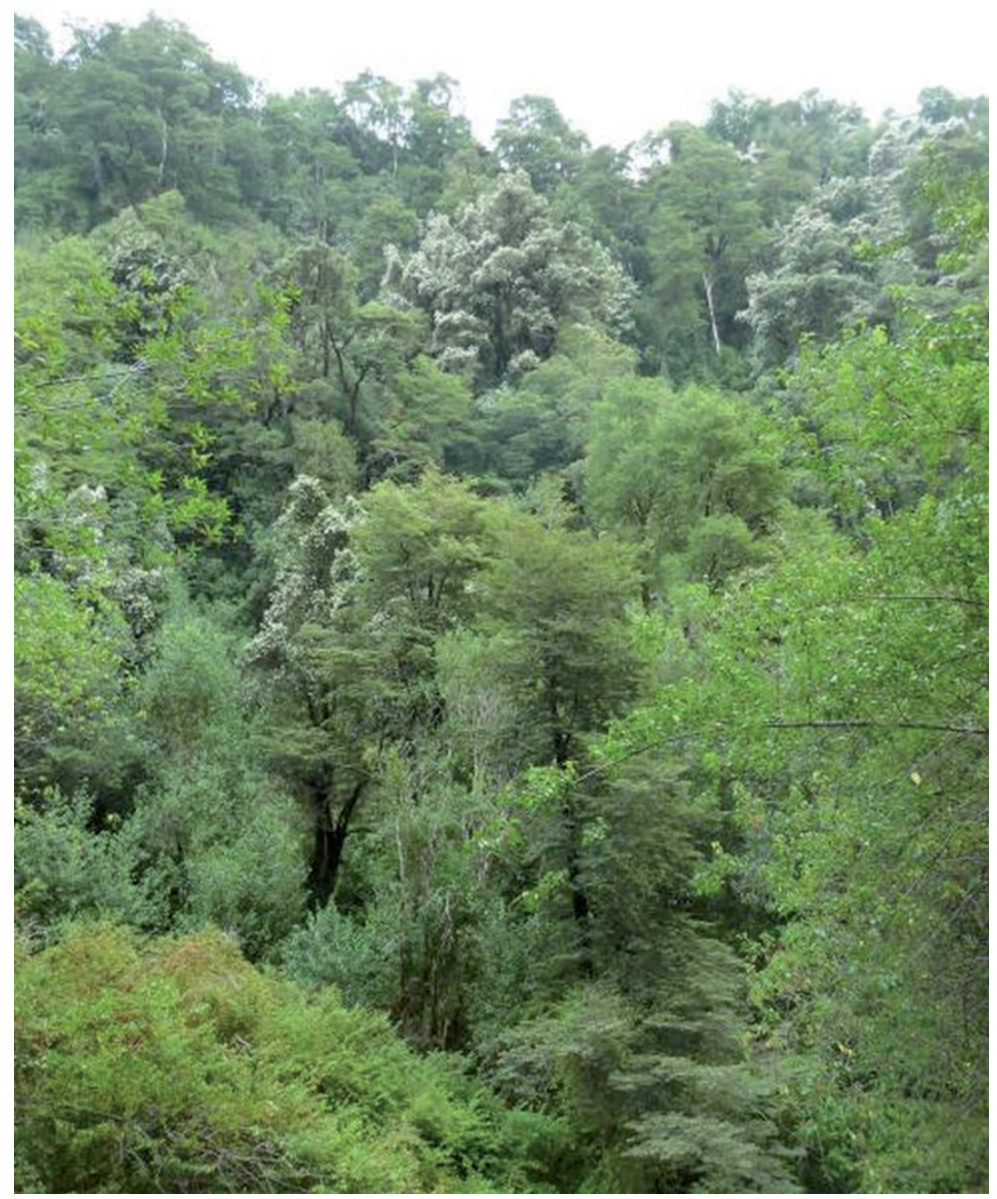

Figura 2. Bosque de tipo valdiviano con Eucryphia cordifolia (Cunoniaceae), el árbol con flores blancas.

FIGURE 2. Valdivian type forest, with Eucryphia cordifolia (Cunoniaceae), the white flowered tree. 


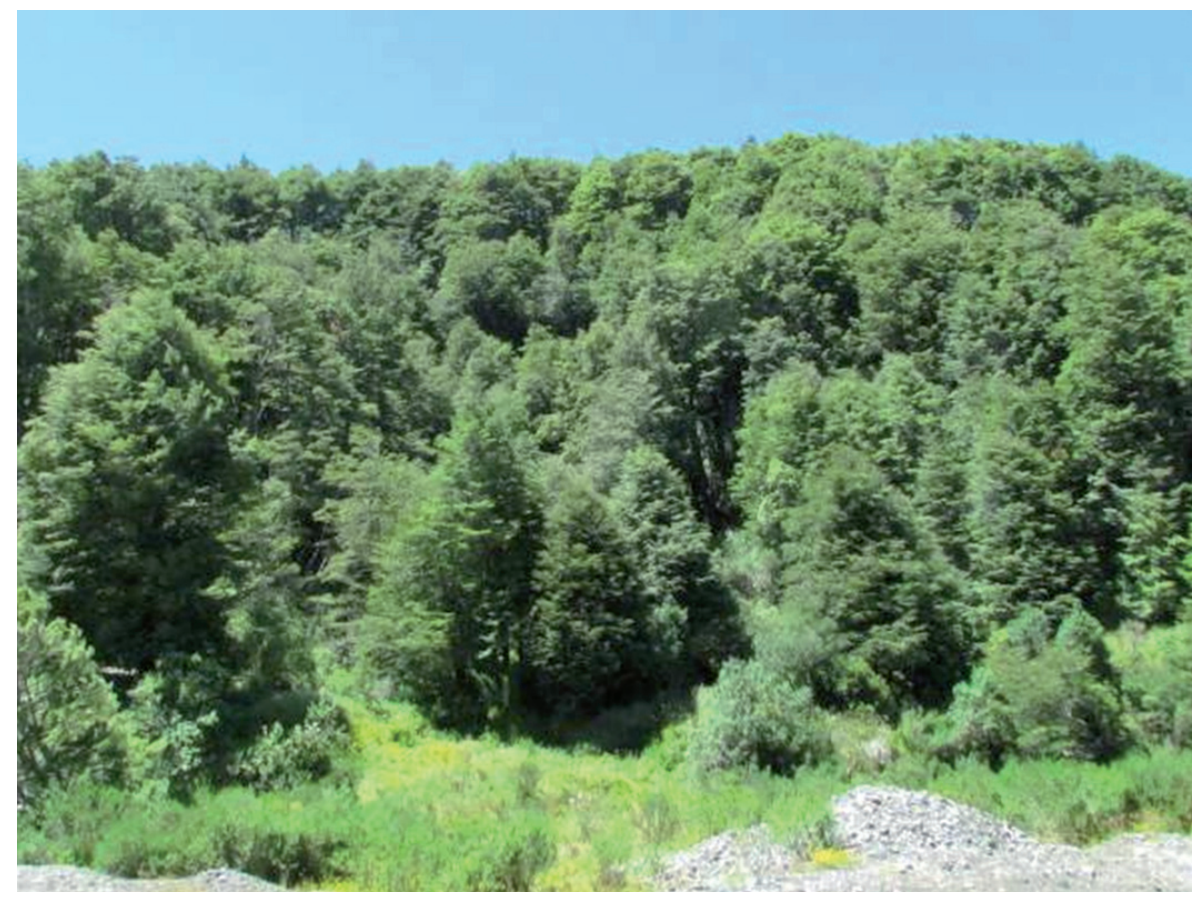

Figura 3. Bosque de Nothofagus dombeyi (Nothofagaceae).

FIGURE 3. Nothofagus dombeyi (Nothofagaceae) forest.

Bosque de NothofaGUS PUMILIO (LENGA): bosque caducifolio (Fig. 4); comunidad de altura donde Nothofagus pumilio es el único árbol dominante, pero presenta un sotobosque con arbustos tales como Drimys andina, Myrceugenia chrysocarpa, Maytenus disticha y Berberis serrato-dentata. En la reserva se encuentra tanto en el volcán MochoChoshuenco como en el camino a Puerto Pirehueico, en las estribaciones occidentales de la cordillera de Arquilhue (cerro
Piedra del Encanto), en ambos casos, por sobre los $1.000 \mathrm{~m}$ de altitud. Por la composición de la flora corresponde con la asociación de Nothofagus pumilio-Drimys winteri var. andina, de la formación del bosque caducifolio altoandino húmedo (Gajardo 1994). La clasificación fitosociológica lo ubica, a su vez, en la asociación Anemono-Nothofagetum pumilionis Oberdorfer.

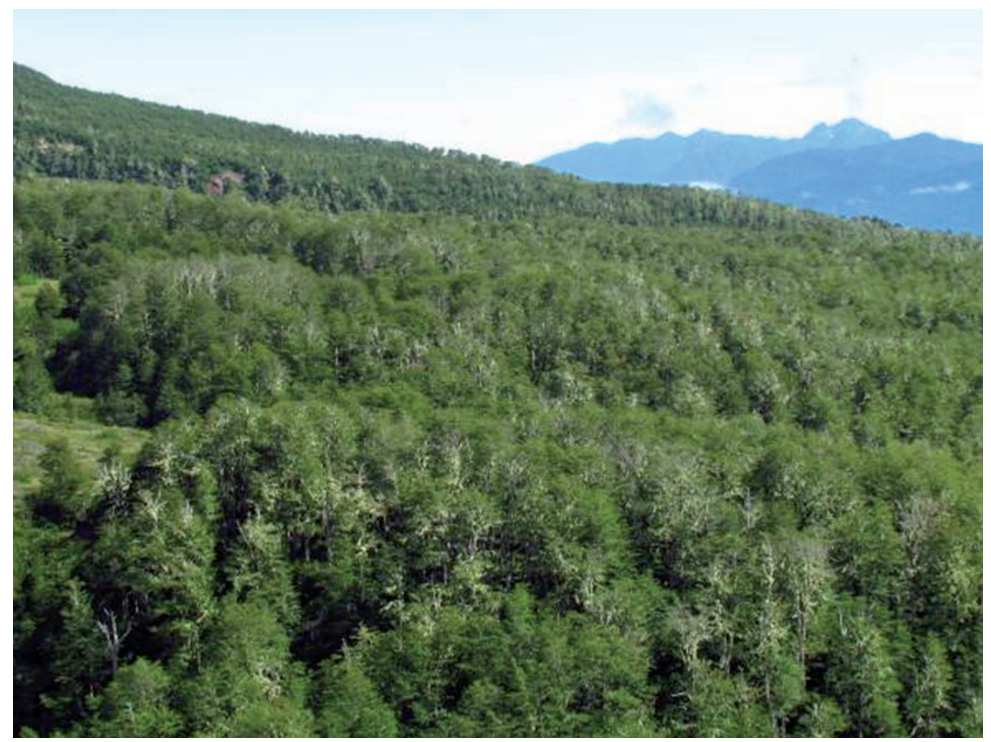

Figura 4. Bosque de Nothofagus pumilio (Nothofagaceae).

FIGURE 4. Nothofagus pumilio (Nothofagaceae) forest. 
Bosque DE Nothofagus ANTARCTICA (ÑIRRe): bosque caducifolio ubicado exclusivamente en la pampa Pilmaiquén, un sitio de acumulación de nieve y posiblemente un área de muy bajas temperaturas. Los ñirres tienen un hábito de árbol y alcanzan hasta $10 \mathrm{~m}$ de altura, aparecen también Lomatia hirsuta, Embothrium coccineum y en los sitios más húmedos, Discaria chacaye. En los claros y en el sotobosque se encuentra un pastizal de Antoxanthum juncifolium (Fig. 5). De acuerdo con lo que se ha informado por pobladores antiguos del área, la pampa se quemaba para destinarla al pastoreo, hoy después de un par de décadas de exclusión, la comunidad tiene un franco carácter de bosque. Los bosques de ñirre no encuentran un símil en la literatura pues son tratados, en general como matorrales; pero, por la composición de su flora en la reserva se pueden relacionar con la asociación de Nothofagus antarctica, perteneciente a la formación del matorral caducifolio altomontano (Gajardo 1994).
MatorRal SUBANDinO: comunidades de altura con cobertura densa, formada por arbustos como Adesmia emarginata, Chiliotrichum diffusum, Discaria chacaye (ecotipo achaparrado), Embothrium coccineum (ecotipo caducifolio), Escallonia alpina, Gaultheria poeppigii, Ovidia andina y Senecio chionophilus, entre otros; en los claros se encuentran pastizales de Anthoxanthum juncifolium, Festuca thermarum y Poa obvallata (Fig. 6). Su distribución en Huilo Huilo es similar a la del bosque de lenga con el que viene en contacto en torno a los 1100-1200 m s.n.m.; se encuentra hasta los $1.500 \mathrm{~m}$ de altitud. La comunidad es afín a la asociación de Discaria chacaye y Berberis empetrifolia propuesta por Becerra y Cruz (2000) para la cordillera de Malalcahuello (IX Región) y correspondería, según Luebert \& Pliscoff (2006), al piso del matorral bajo templado andino de Discaria chacaye y Berberis empetrifolia.

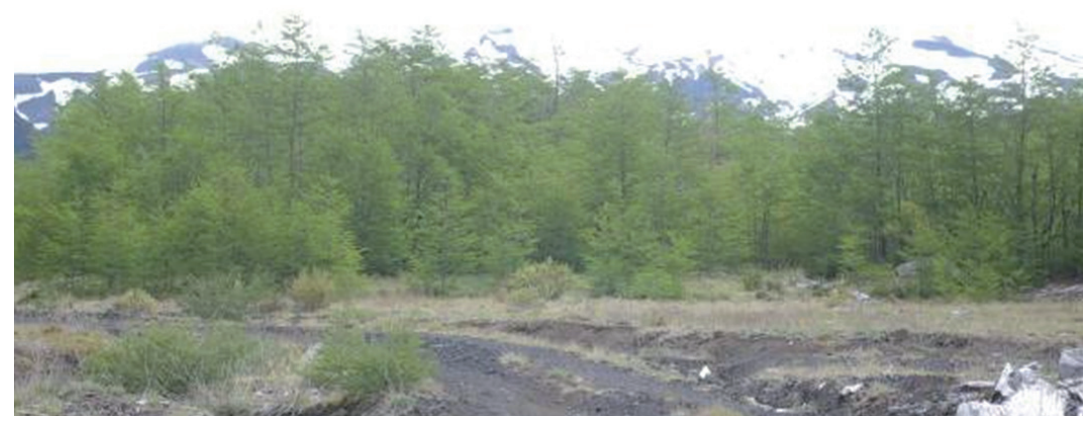

Figura 5. Bosque de Nothofagus antarctica (Nothofagaceae).

FIGURE 5. Nothofagus antarctica (Nothofagaceae) forest.

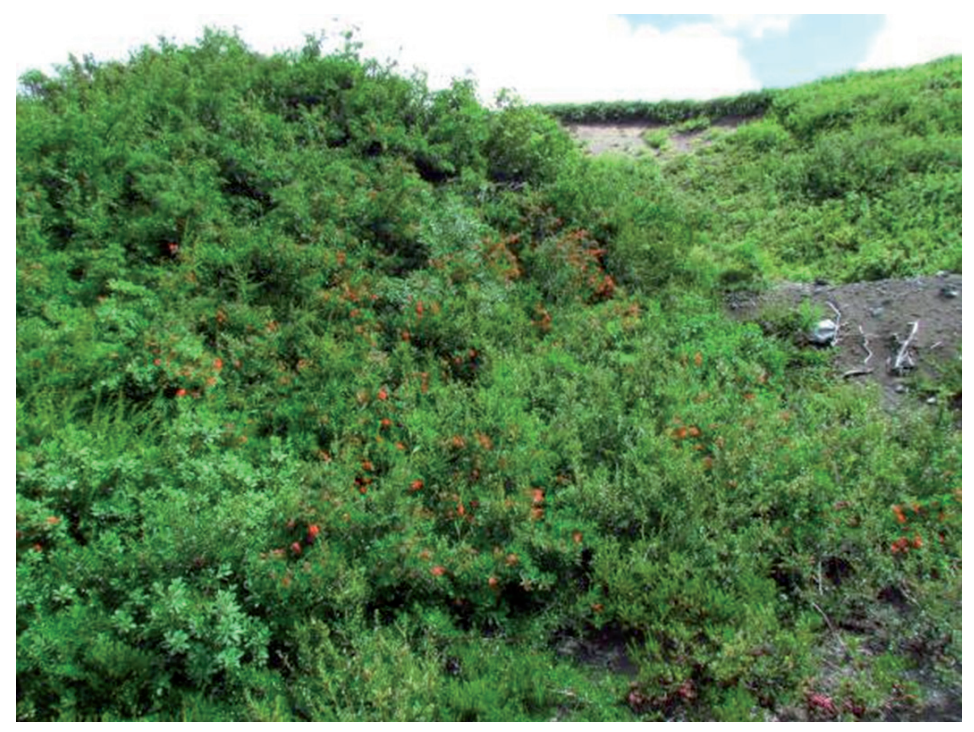

Figura 6. Matorral subandino con Embothrium coccineum (Proteaceae), arbusto con flores rojas.

FIGURE 6. Subandean scrub with Embothrium coccineum (Proteaceae), the red flowered shrub. 
MatorRal bajo altoAndino: comunidad donde dominan arbustos como Adesmia longipes, Nassauvia lagascae, Nassauvia revoluta y Senecio bipontinus, y una gramínea en champa, Poa obvallata. La cobertura de la vegetación es muy baja (Fig. 7). Este tipo de vegetación se encontró sólo en el volcán Mocho-Choshuenco, sobre $1.450 \mathrm{~m}$ de altitud y forma allí el límite superior de la vegetación, el que se encuentra aproximadamente entre los 1.550 y los $1.650 \mathrm{~m}$ s.n.m.La comunidad, por su composición de especies, es afín a la asociación de Adesmia longipes y Azorella incisa, perteneciente a la formación de la estepa patagónica de Aisén (Gajardo 1994). Luebert \& Pliscoff (2006) incluyen a la asociación en el piso del matorral bajo templado andino de Adesmia longipes y Senecio bipontinus.

Humedales: en la reserva se encuentran humedales de diferentes tipos, pero tienen en común la presencia importante de ciperáceas, juncáceas y gramíneas. Se desarrollan a lo largo de los esteros y los ríos (ej. valle del Pillanleufú), en áreas pantanosas (ej. Los Planchados) y en torno a la laguna Trarolafquén (Fig. 8), un cuerpo de agua con nivel inter-estacional muy variable que se encuentra en la pampa Pilmaiquén, cuyas riberas tienen una flora muy particular.

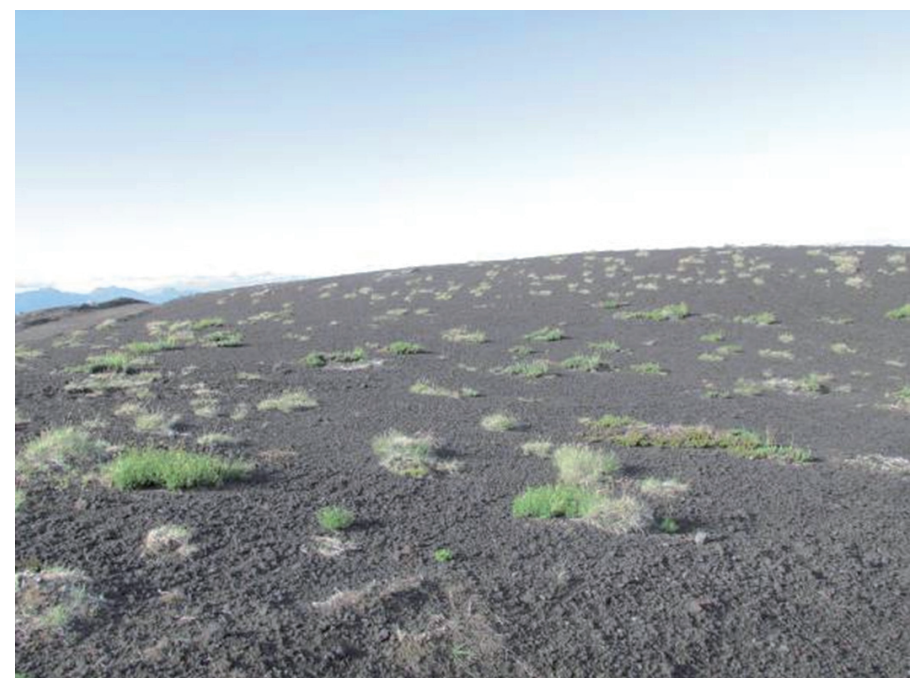

Figura 7. Herbazal andino con Senecio bipontinus (Asteraceae) y Poa obvallata (Poaceae).

Figure 7. Andean grassland with Senecio bipontinus (Asteraceae) and Poa obvallata (Poaceae).

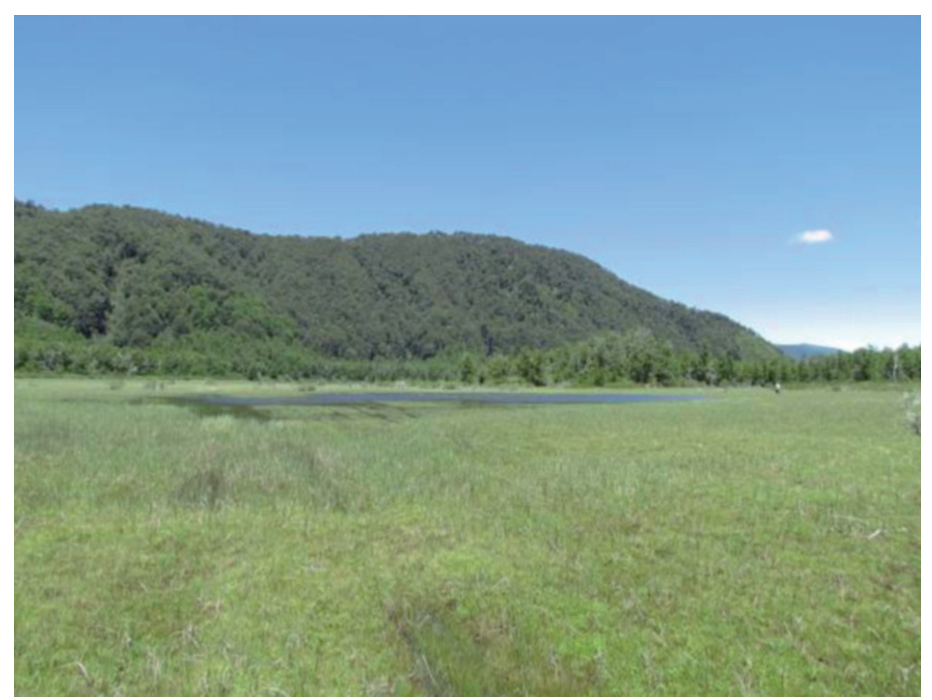

Figura 8. Laguna Trarolafquén.

FIGURE 8. Trarolafquen lagoon. 
Praderas ANTRopogénicas: se ubican en sitios que se utilizaron para la agricultura, ganadería o instalación de faenas forestales; también, a lo largo de los caminos, en sitios despejados (Fig. 9). Están dominadas por especies alóctonas como Holcus lanatus, Dactylis glomerata y Anthoxanthum odoratum, la única gramínea nativa frecuente en ellos es Paspalum dasypleurum.

\section{CAMPAÑAS EN TERRENO}

Se llevaron a cabo seis visitas a terreno, desarrolladas entre los meses de noviembre y febrero, de los años 2010, 2011 y 2012, las que tuvieron una duración de 4-6 días cada una. Se efectuaron numerosos recorridos dirigidos a los diferentes tipos de comunidades vegetales que se describen más arriba. En el área existen numerosos caminos forestales antiguos que facilitaron el muestreo. Se recolectaron y herborizaron cerca de 800 números, de los que una parte importante ha sido depositada en el Herbario del Departamento de Botánica de la Universidad de Concepción (CONC) y en otros herbarios como MIN, US y PRA.

\section{DETERMINACIÓN DE LAS PLANTAS}

Las determinaciones de las plantas fueron hechas por los autores con base en la experiencia de campo. Aquellas que no fue posible identificar en terreno se llevaron a gabinete y se determinaron con la ayuda de la literatura disponible pertinente, particularmente Flora Patagonica (Correa 19841999) y los volúmenes de Flora de Chile (Marticorena \& Rodríguez 1985-2012).

Para determinar algunas especies de los grupos o géneros que se mencionan, se contó con la colaboración de los siguientes especialistas: M. Baeza (Festuca, Rytidosperma), L. Clark (Chusquea), M. Costea (Cuscuta),
V. Finot (Festuca, Trisetum), R. Guaglianone (Eleocharis), P. Hechenleitner (Ribes, Vicia), J. Kirschner (Juncaceae), R. Rodríguez (Pteridófita), R. Soreng (Poa) y G.A. Wheeler (Carex, Uncinia), a los que los autores agradecen su buena disposición.

\section{CARACTERIZACIÓN DE LA FLORA}

La nomenclatura de las especies sigue en la mayor parte de los casos a Zuloaga et al. (2008 en adelante); pero también, las propuestas de las publicaciones de Marticorena \& Quezada (1985) y del proyecto Flora de Chile (Marticorena \& Rodríguez 1995-2012). En ciertos casos se prefirió conservar la nomenclatura "antigua" de las especies por estar más difundida, por poco uso de alguna propuesta nueva en la literatura florística o por falta de convencimiento por parte de los autores sobre alguna de ellas. La nomenclatura de las familias sigue en parte las ideas de la APG III (Stevens 2001, en adelante), sin embargo, se conserva a las Scrophulariaceae dentro de los límites clásicos, con excepción del reconocimiento de Calceolariaceae.

La presentación de las especies en el Anexo 1 está organizada siguiendo la secuencia Pteridophyta, Pinophyta (Gimnospermas) y Magnoliophyta (Angiospermas); en el caso de estas últimas, se sigue el orden "clásico", separando y ordenando las familias en dicotiledóneas sensu lato que incluye las angiospermas primitivas -clados ANITA y magnolídeas- (Stevens 2001 en adelante) y monocotiledóneas.

A cada especie se le ha asignado la forma de vida de Raunkiaer tomando la descripción de ellas que figura en Braun-Blanquet (1979); la nomenclatura de las traducciones sigue a Font Quer (1982).

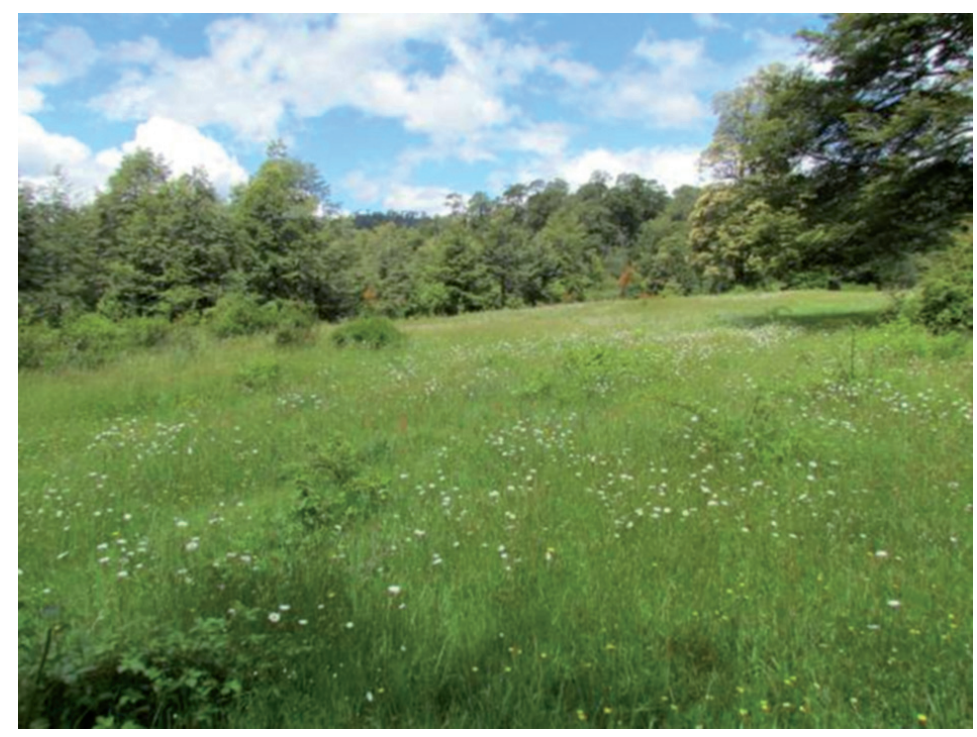

Figura 9. Pradera antropogénica, pampa Bandurrias, cerca de la entrada a la reserva.

FIGURE 9. Anthropogenic grassland at pampa Bandurrias near the entrance of the reserve. 
A cada especie se le atribuyó un origen fitogeográfico, a saber: endémica de Chile, endémica de Chile y Argentina, nativa no endémica y alóctona asilvestrada, además se indica la distribución de la especies en las regiones administrativas del país. Los datos fueron tomados de Matthei (1996) y de Zuloaga et al. (2008 en adelante), con algunas modificaciones.

Se indica el estado de conservación de aquellas especies que han sido clasificadas en los diversos procesos de asignación de categorías de amenaza por parte del Estado de Chile. De este modo se citan las especies propuestas por CONAMA y por el Ministerio del Medio Ambiente (MINSEGPRES 2007-2013), aquellas que figuran en el Libro Rojo de la Flora de Chile (Benoit 1989) y las del Boletín $\mathrm{N}^{\circ} 47$ del Museo Nacional de Historia Natural que contiene los trabajos sobre pteridófita amenazadas de Baeza et al. (1998) y de monocotiledóneas con flores vistosas de Ravenna et al. (1998). En algunos casos se citan propuestas de organismos internacionales de conservación o de algunos especialistas (ej. Zizka et al. (2009) para Bromeliaceae y Hechenleitner et al. (2005)).

\section{RESULTADOS}

RiQUEZA Y COMPOSICIÓN DE LA FLORA VASCULAR

La riqueza de la flora vascular en el área de estudio alcanza a 406 especies, éstas se distribuyen en 95 familias y 230 géneros (Catálogo en Anexo 1).
Las familias con más de diez especies en el área de estudio son: Asteraceae, con 60 (14,8\% del total); Poaceae, con 43 (10,6\%); Cyperaceae, con 21 (5,1\%), Hymenophyllaceae con 16 (3,9\%); Scrophulariaceae sensu lato, con 13 (3,2\%); Juncaceae y Fabaceae, con 12 (2,9\%); Rosaceae, con 11 (2,7\%) y Ranunculaceae, con 10 $(2,4 \%)$ (Tabla I). Entre los géneros destacan por riqueza, Hymenophyllum con 13 especies; Carex y Senecio con 9; Agrostis, Baccharis y Ranunculus con 7; Blechnum, con 6 y finalmente con 5, figuran Acaena, Calceolaria, Epilobium, Escallonia, Gamochaeta, Gaultheria, Nothofagus, Ourisia y Uncinia (Tabla II).

\section{ORIGEN FITOGEOGRÁFICO}

La mayor parte de las especies que se registra en el área corresponde a nativas: 340 (83,7\%); las alóctonas asilvestradas alcanzan a $67(16,3 \%)$. Entre las nativas predominan las plantas endémicas de Chile y Argentina, con 266 especies (78,2\% del total de nativas); seguidas por las nativas no endémicas ni de Chile ni de Argentina, con $54(15,9 \%)$ y las endémicas de Chile, con 20 (5,9\%) (Tabla III).

Entre las especies endémicas de Chile, la mayor parte tiene rangos de distribución que alcanza a varias regiones administrativas del país (Tabla IV), con excepción de Elaphoglossum fonkii (Lomariopsidae, Pteridophyta) que se encuentra exclusivamente en las regiones de Los Ríos y de Los Lagos, y Ercilla syncarpellata (Phytolaccaceae) que se ha recolectado entre las regiones de La Araucanía y de Los Lagos.

TABLa I. Flora vascular de la Reserva Biológica de Huilo Huilo (Región de Los Ríos, Chile): familias con mayor número de taxones. EC: Endémicas de Chile; ECA: Endémicas de Chile y Argentina; NNE: Nativas no endémicas; Nat: Nativas=EC+ECA+NNE; Al: Alóctonas asilvestradas.

TABle I. Vascular flora at Huilo Huilo Reserve (Región de Los Ríos, Chile): larger botanical families. EC: Endemic to Chile; ECA: Endemic to Chile and Argentina; NNE: Native non endemic; Nat: Native=EC+ECA+NNE; Al: Alien.

\begin{tabular}{lrrrrrr}
\hline FAMILIA & EC & ECA & NNE & NAT & AL & TOTAL \\
& & & & & & \\
\hline Asteraceae & 0 & 43 & 4 & 47 & 13 & 60 \\
Poaceae & 0 & 23 & 5 & 28 & 15 & 43 \\
Cyperaceae & 0 & 12 & 9 & 21 & 0 & 21 \\
Hymenophyllaceae & 2 & 14 & 0 & 14 & 0 & 16 \\
Scrophulariaceae & 0 & 8 & 1 & 9 & 4 & 13 \\
Juncaceae & 1 & 2 & 8 & 10 & 1 & 12 \\
Fabaceae & 0 & 5 & 1 & 6 & 6 & 12 \\
Rosaceae & 0 & 7 & 1 & 8 & 3 & 11 \\
Otras familias & 17 & 157 & 25 & 199 & 24 & 218 \\
\hline
\end{tabular}


Flora vascular de la Reserva Biológica Huilo Huilo: TeILLIER, S. ET AL.

TABla II. Flora vascular de la Reserva Biológica de Huilo Huilo (Región de Los Ríos, Chile): géneros con mayor número de taxones. EC: Endémicas de Chile; ECA: Endémicas de Chile y Argentina; NNE: Nativas no endémicas; Nat: Nativas=EC+ECA+NNE; Al: Alóctonas asilvestradas.

TABLE II. Vascular flora at Huilo Huilo Reserve (Región de Los Ríos, Chile): larger botanical genera. EC: Endemic to Chile; ECA: Endemic to Chile and Argentina; NNE: Native non endemic; Nat: Native=EC+ECA+NNE; Al: Alien.

\begin{tabular}{lrrrrrr}
\hline GéNERo & EC & ECA & NNE & NAT & AL & TOTAL \\
\hline Hymenophyllum & 1 & 12 & 0 & 0 & 13 & 13 \\
Carex & 0 & 8 & 1 & 9 & 0 & 9 \\
Senecio & 0 & 9 & 0 & 9 & 0 & 9 \\
Agrostis & 0 & 3 & 0 & 3 & 4 & 7 \\
Baccharis & 0 & 6 & 1 & 7 & 0 & 7 \\
Blechnum & 0 & 3 & 3 & 6 & 0 & 6 \\
Acaena & 0 & 4 & 1 & 5 & 0 & 5 \\
Calceolaria & 1 & 4 & 0 & 5 & 0 & 5 \\
Epilobium & 0 & 3 & 2 & 5 & 0 & 5 \\
Escallonia & 0 & 5 & 0 & 5 & 0 & 5 \\
Gamochaeta & 0 & 4 & 1 & 5 & 0 & 5 \\
Gaultheria & 0 & 5 & 0 & 5 & 0 & 5 \\
Nothofagus & 0 & 5 & 0 & 5 & 0 & 5 \\
Ourisia & 0 & 5 & 0 & 5 & 0 & 5 \\
Uncinia & 0 & 2 & 3 & 5 & 0 & 5 \\
\hline
\end{tabular}

TABla III. Flora vascular de la Reserva Biológica Huilo Huilo (Región de Los Ríos, Chile): distribución de las especies nativas según origen fitogeográfico.

TABLE III. Vascular flora at Huilo Huilo Reserve (Región de Los Ríos, Chile): distribution of native species according to phytogeographical origin.

\begin{tabular}{lcc}
\hline Origen fitogeográfico & EspeCies $\left(\mathrm{N}^{\circ}\right)$ & $\begin{array}{c}\text { \% DEL tOtAl de } \\
\text { NAtivas }\end{array}$ \\
\hline Endémicas de Chile & 20 & 5,9 \\
Endémicas de Chile y Argentina & 266 & 78,2 \\
Nativas no endémicas de Chile ni Argentina & 54 & 15,9 \\
\hline Total & 340 & 100 \\
\hline
\end{tabular}


TABla IV. Flora de la Reserva Biológica Huilo Huilo (Región de Los Ríos, Chile): especies endémicas de Chile, distribución en el país.

TABLE IV. Vascular flora at Huilo Huilo Reserve (Región de Los Ríos, Chile): plant species endemic to Chile, geographical distribution.

\begin{tabular}{|c|c|c|}
\hline FAMILIA & ESPECIE O SUBESPECIE & $\begin{array}{l}\text { DistribuCIÓN (REGIONES } \\
\text { ADMINISTRATIVAS DE CHILE) }\end{array}$ \\
\hline Asteraceae & Acrisione cymosa (J. Remy) B. Nord. & XIV-XI \\
\hline Bromeliaceae & Fascicularia bicolor (Ruiz \& Pav.) Mez & $\mathrm{V}-\mathrm{X}$ \\
\hline Bromeliaceae & Greigia pearcei $\mathrm{Mez}$ & VIII-X \\
\hline Calceolariaceae & Calceolaria valdiviana Phil. & VIII-X \\
\hline Francoaceae & Francoa appendiculata Cav. & $\mathrm{V}-\mathrm{X}$ \\
\hline Gesneriaceae & Sarmienta scandens (J.D. Brandis ex Molina) Pers. & (IV) VII-X \\
\hline Gleicheniaceae & Gleichenia squamulosa (A.N. Desv.) Moore & VII-XI \\
\hline Hymenophyllaceae & Trichomanes exsectum Kunze & VII-X \\
\hline Hypericaceae & Hypericum caespitosum Cham. \& Schltdl. & VII-X \\
\hline Iridaceae & Libertia tricocca Phil. & VII-XIV \\
\hline Iridaceae & Olsynium junceum (E. Mey. ex C. Presl) Goldblatt ssp. depauperatum & VIII-X \\
\hline Iridaceae & Sisyrinchium pearcei Phil. & IX-XII \\
\hline Juncaceae & Luzula tristachya Desv. & IV-X \\
\hline Lomariopsidaceae & Elaphoglossum fonkii (Phil.) T. Moore & $\mathrm{XIV}-\mathrm{X}$ \\
\hline Luzuriagaceae & Luzuriaga polyphylla (Hook.) J.F. Macbr. & VII-XII \\
\hline Philesiaceae & Lapageria rosea Ruiz et Pav. & IV-X \\
\hline Phytolaccaeae & Ercilla syncarpellata Nowicke & IX-X \\
\hline Urticaceae & Pilea elliptica Hook. f. & IX-XI \\
\hline Winteraceae & Drimys andina (Reiche) R. Rodr. \& Quezada & VIII-X \\
\hline
\end{tabular}

Las pteridófitas, incluyendo Lycopodium, Equisetum y helechos, alcanzan a 45 especies, 3 endémicas de Chile, 34 de Chile y Argentina, y 8 nativas, no endémicas. Las gimnospermas incluyen sólo 3 especies, 1 endémica de Chile y Argentina (Saxegothaea conspicua) y 2 alóctonas asilvestradas; las angiospermas comprenden, a su vez, a 361 especies, de las que 268 son dicotiledóneas s.l. y 93 monocotiledóneas. Entre las dicotiledóneas s.l., 184 especies son endémicas de Chile y Argentina, 10 de Chile y 24 son nativas no endémicas, hay además 48 alóctonas asilvestradas; entre las monocotiledóneas se encuentran 47 endémicas de Chile y Argentina, 8 de Chile, 22 nativas no endémicas y 16 alóctonas asilvestradas.

En relación con las especies alóctonas asilvestradas o introducidas, desde el punto de vista taxonómico destacan en riqueza de especies las Poaceae con 15 especies que representan un $34 \%$ de las especies de la familia en Huilo Huilo y las Asteraceae, con 13, que corresponden a un $21,6 \%$; cantidades menores, pero con altos porcentajes del total de especies se registran entre las Polygonaceae con 6 de 7 especies (83\%); las Caryophyllaceae con 5 de $8(62 \%)$ y las Fabaceae con 6 de $12(50 \%)$. Los géneros con más especies advenas son Agrostis con 4, Trifolium y Polygonum con 3.
Nuevos REgistros

Se encontró una especie no registrada previamente para Chile: Primula comberi (Primulaceae, Fig. 10) y una variedad: Oenothera stricta var. altissima (Onagraceae). Se amplía la distribución de Trichomanes exsectum (Hymenophyllaceae) que no era conocido para la cordillera de los Andes (Macaya-Berti et al. 2012), la de Lycopodium albofii (Lycopodiaceae) con un nuevo límite norte de distribución, la de Epilobium densifolium (Onagraceae), también con un nuevo límite norte y la de Ranunculus hydrophilus (Ranunculaceae), primera mención para la Región de Los Ríos.

\section{Formas DE VIDA DE RAUNKIAER}

El análisis muestra que predominan los hemicriptófitos con un $61 \%$ de las especies (248); seguidos por los fanerófitos, que incluyen árboles y trepadoras leñosas con 11,3\% (46); a continuación figuran los nanofanerófitos, arbustos de más de $50 \mathrm{~cm}$ de altura, con $11 \%$ (45); valores menores muestran los terófitos, 4,9\%; los geófitos, 4,4\%; los caméfitos, 3,9\%; los epífitos, que agrupan a los epífitos obligados y a los parásitos leñosos, y los hidrófitos (Tabla V); el detalle de la forma dominante, los hemicriptófitos, muestra que entre ellos los principales grupos son los en roseta $(31,8 \%)$, los 
cespitosos $(28,5 \%)$, los escapíferos (21\%) y los repentes $(9,9 \%)$ (Tabla V).

Entre las nativas predominan los hemicriptófitos $(60,3 \%)$; seguidos por los fanerófitos (12,3\%) y los nanofanerófitos $(11,7 \%)$. Si se analiza el espectro de las formas de vida entre las alóctonas asilvestradas, la forma de crecimiento dominante es también la de los hemicriptófitos
$(65,1 \%)$; si se lo compara con el de las nativas, se observa que la diferencia principal es la proporción de los terófitos, que agrupan a las hierbas anuales, que sobrepasan en riqueza a los terófitos nativos y alcanzan a un 19,6\% de las alóctonas, superando ampliamente el 2,1\% que alcanzan entre las nativas (Tabla VI).

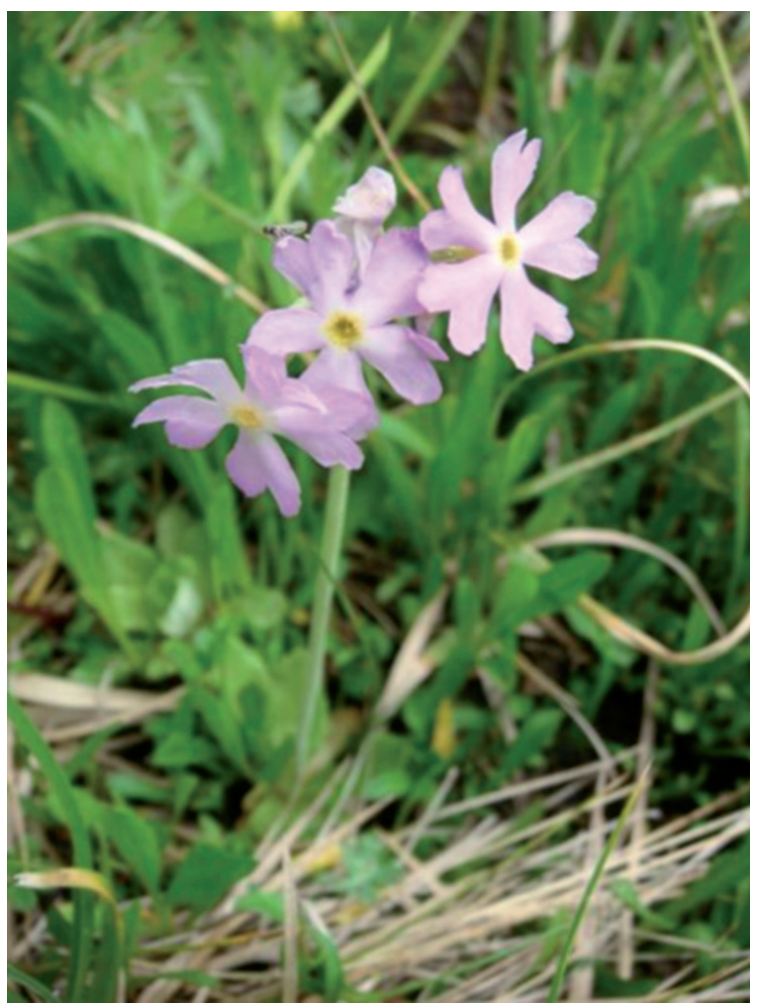

Figura 10. Primula comberi (Primulaceae), una nueva especie para Chile.

Figure 10. Primula comberi (Primulaceae), a new species for the chilean flora.

Tabla V. Flora de la Reserva Biológica Huilo Huilo (Región de Los Ríos, Chile): distribución de especies por forma de vida de Raunkiaer. EC: Endémicas de Chile; ECA: Endémicas de Chile y Argentina; NNE: Nativas no endémicas; Nat: Nativas=EC+ECA+NNE; Al: Alóctonas asilvestradas.

TABle V. Vascular flora at Huilo Huilo Reserve (Región de Los Ríos, Chile): species by Raunkiaer's life forms. EC: Endemic to Chile; ECA: Endémic to Chile and Argentina; NNE: Native non endémic; Nat: Native=EC+ECA+NNE; Al: Alien.

\begin{tabular}{lrrrrrr}
\hline Forma DE VIDA & EC & ECA & NNE & NAT & AL & TOTAL \\
\hline Caméfito & 0 & 16 & 0 & 16 & 0 & 16 \\
Epífito & 1 & 8 & 0 & 9 & 0 & 9 \\
Fanerófito & 4 & 38 & 0 & 42 & 4 & 46 \\
Geófito & 3 & 11 & 3 & 205 & 43 & 248 \\
Hemicriptófito & 10 & 150 & 45 & 4 & 0 & 4 \\
Hidrófito & 0 & 1 & 2 & 70 & 5 & 45 \\
Nanofanerófito & 2 & 36 & 1 & & 13 & 20 \\
Terófito & 0 & 6 & & & & \\
\hline
\end{tabular}


TABla VI. Flora de la Reserva Biológica de Huilo Huilo (Región de Los Ríos, Chile): tipos de hemicriptófitos, la forma de vida dominante entre las especies EC: Endémicas de Chile; ECA: Endémicas de Chile y Argentina; NNE: Nativas no endémicas; Nat: Nativas=EC+ECA+NNE; $\mathrm{Al}$ : Alóctonas asilvestradas.

Table VI. Vascular flora at Huilo Huilo Reserve (Región de Los Ríos, Chile): hemicryptophyta types. EC: Endemic to Chile; ECA: Endémic to Chile and Argentina; NNE: Native non endémic; Nat: Native=EC+ECA+NNE; Al: Alien.

\begin{tabular}{lrrrrrr}
\hline Forma DE VIDA & EC & ECA & NNE & NAT & AL & TOTAL \\
\hline Bienales & 0 & 0 & 0 & 0 & 2 & 2 \\
Cespitosos & 1 & 35 & 21 & 57 & 12 & 69 \\
En roseta & 5 & 54 & 6 & 65 & 12 & 77 \\
Escandentes & 0 & 4 & 2 & 6 & 0 & 6 \\
Escapíferos & 0 & 29 & 10 & 39 & 12 & 51 \\
Repentes & 2 & 13 & 4 & 19 & 5 & 24 \\
Rizomatosos & 2 & 15 & 2 & 19 & 0 & 19 \\
\hline
\end{tabular}

ESPECIES EN CATEGORÍAS DE CONSERVACIÓN

Se encontraron 18 especies que han sido incluidas en alguna categoría de conservación por alguna de las instancias que han producido clasificaciones de especies (Tabla VII). Greigia pearcei, si bien se clasificó preliminarmente como insuficientemente conocida por Hoffmann \& Flores (1989), Zizka et al. (2009) la cambiaron a "en peligro crítico", propuesta que se recoge en este trabajo. 13 especies han sido clasificadas como vulnerables, de las que Elaphoglossum fonkii, Fascicularia bicolor, Libertia tricocca y Trichomanes exsectum son especies endémicas de Chile. De las especies clasificadas por los procesos oficiales por CONAMA y el Ministerio del Medio Ambiente, se registró a Persea lingue clasificado como de "preocupación menor" en el área sur de su distribución (Ministerio Secretaría General de la Presidencia 2011). De las 19 especies endémicas de Chile, 11 han sido objeto de alguna clasificación.

\section{DISCUSIÓN}

RIQUEZA EN TÉRMINOS COMPARATIVOS CON OTRAS LOCALIDADES DEL BOSQUE TEMPLADO AUSTRAL

Para comparar las riquezas de varias localidades que contienen comunidades de bosque austral templado, se utilizó un "índice de biodiversidad", donde se relacionan la superficie del área de estudio con la riqueza de especies, mediante la fórmula: $\mathrm{IB}=\mathrm{ni} / \mathrm{ln} \mathrm{Ai}$, donde $n i$ es el número de taxa y $A i$, la superficie del área del estudio (Zuloaga et al. 1999). El índice de biodiversidad para la Reserva Biológica Huilo Huilo es de 41, y se ubica bajo los índices del Monumento Natural Contulmo (Región de la Araucanía, Chile) y del Parque Nacional Nahuel Huapi en Argentina, superando a los parques nacionales Puyehue (Región de
Los Lagos, Chile) y Laguna San Rafael (Región de Aysen, Chile), a las reservas nacionales Malalcahuello y Tolhuaca, ambas situadas en la Región de La Araucanía, Chile, y a un levantamiento de la flora vascular de las cuencas de los ríos Baker y Pascua (Región de Aysen). Los resultados se muestran en la Tabla VIII.

REPRESENTATIVIDAD DE LA FLORA VASCULAR EN EL MARCO DEL BOSQUE LLUVIOSO TEMPLADO AUSTRAL

Arroyo et al. (1995) publicaron una lista de especies leñosas que se encuentran en los bosques lluviosos templados australes; a continuación se analizará la representatividad de la flora vascular de la Reserva Biológica de Huilo Huilo en relación con dicha propuesta. De acuerdo con los datos de la Tabla IX se obtiene que $82,3 \%$ de las familias de los bosques australes templados está representado en el área de estudio, destacando un 91\% de las de Pteridophyta y un 83,8\% de las de dicotiledóneas (s.l.). En relación con los géneros, en el área se encuentra un $77 \%$ de los presentes en este tipo de bosque; entre éstos destacan los de las Pteridophyta con un $82,6 \%$ y los de dicotiledóneas (s.l.) que alcanzan a casi un $76 \%$. De los géneros endémicos de estos bosques que citan Arroyo et al. (1995), se registran los siguientes 19: Acrisione, Aextoxicon, Amomyrtus, Asteranthera, Boquila, Campsidium, Elytropus, Embothrium, Fonkia (Gratiola), Hymenoglossum, Lapageria, Laureliopsis, Luma, Misodendron, Mitraria, Myoschilos, Ovidia, Sarmienta y Saxegothaea (Tabla X). Los menores porcentajes de representatividad, tanto para familias como para géneros, corresponden a las Pinophyta (coníferas), de las que sólo una especie nativa se encuentra en el área de estudio: Saxegothaea conspicua.

Se registró un alto grado de endemismos de Chile y Argentina, casi un 80\%, y uno bajo al nivel de Chile (cerca 
de 6\%), lo que da cuenta de que las especies del área corresponden en su mayoría a elementos característicos del bosque lluvioso templado austral cuya área de distribución comprende a ambos países.

Las plantas alóctonas asilvestradas alcanzan un porcentaje de casi un $16 \%$, alto si se lo compara con el $11 \%$ al nivel nacional (Marticorena 1990), lo que está ligado a la historia de uso de suelo del predio, que fue agrícola en la parte baja y ganadero-forestal en la más alta.

\section{RiQUEZA POR TIPO DE COMUNIDAD VEGETAL}

Un análisis de las especies por comunidad vegetal muestra que entre los tipos de bosque el valdiviano es el más rico con 136 de las 337 nativas del área de estudio; lo siguen los bosques de Nothofagus dombeyi, con 96; el de Nothofagus antarctica, con 45 y el de Nothofagus pumilio con 34. Dado que la riqueza está relacionada con la superficie, es notable que el bosque de tipo valdiviano, que es uno de los que ocupa la menor superficie tenga la riqueza más alta. Una parte importante de la diferencia, comparando con las otras comunidades de bosque, la constituye su alta riqueza de lianas y helechos. Respecto de este mismo tipo de bosque, hay que resaltar, además, que 13 de las 19 especies endémicas de Chile crecen en ellos y que la mayoría de las especies en categorías de conservación del área también se encuentran en dicha comunidad. Los matorrales subandinos reúnen a 51 especies, mientras que el herbazal andino presenta una riqueza de sólo 32. Las praderas antropogénicas, en su gran mayoría, reúnen apenas a 16 especies nativas, en tanto que las praderas que se desarrollan en condiciones de humedad permanente, a 65 .

TABLA VII. Flora vascular de la Reserva Biológica de Huilo Huilo (Región de Los Ríos, Chile): especies en categorías de conservación.

TABLE VII. Vascular flora at Huilo Huilo Reserve (Región de Los Ríos, Chile): threatened species.

\begin{tabular}{|c|c|c|c|}
\hline FAMILIA & EsPECIE & $\begin{array}{l}\text { CATEGORÍA DE } \\
\text { CONSERVACIÓN }\end{array}$ & FUENTE \\
\hline Aspleniaceae & Asplenium trilobum Cav. & Vulnerable & Baeza et al. (1998) \\
\hline Bromeliaceae & Greigia pearcei $\mathrm{Mez}$ & En peligro crítico & Zizka et al. (2009) \\
\hline Bromeliaceae & Fascicularia bicolor (Ruiz \& Pav.) Mez & Vulnerable & Hoffmann \& Flores (1998) \\
\hline Dennstaedtiaceae & Hypolepis poeppigii (Kunze) Mettenius & Vulnerable & Baeza et al. (1998) \\
\hline Dicksoniaceae & Lophosoria quadripinnata (J.F. Gmel.) C. Chr. & Vulnerable & Baeza et al. (1998) \\
\hline Dryopteridaceae & Megalastrum spectabile (Kaulf.) A.R. Sm. et R.C. Moran & Rara & Baeza et al. (1998) \\
\hline Dryopteridaceae & Polystichum subintegerrimum (Hook. \& Arn.) R. Rodr. & Rara & Baeza et al. (1998) \\
\hline Grammitidaceae & Grammitis magellanica A.N. Desv. & Vulnerable & Baeza et al. (1998) \\
\hline Hymenophyllaceae & Hymenoglossum cruentum (Cav.) K. Presl. & Vulnerable & Baeza et al. (1998) \\
\hline Hymenophyllaceae & Hymenophyllum caudiculatum Mart. & Vulnerable & Baeza et al. (1998) \\
\hline Hymenophyllaceae & Hymenophyllum dicranotrichum (K. Presl.) Sadeb. & Vulnerable & Baeza et al. (1998) \\
\hline Hymenophyllaceae & Hymenophyllum tortuosum Hook. \& Grev. & Vulnerable & Baeza et al. (1998) \\
\hline Hymenophyllaceae & Serpyllopsis caespitosa (Gaudich.) C. Chr. & $\begin{array}{l}\text { Insuficientemente } \\
\text { conocida }\end{array}$ & Baeza et al. (1998) \\
\hline Hymenophyllaceae & Trichomanes exsectum Kunze & Vulnerable & $\begin{array}{c}\text { Rodríguez (1989), Baeza et } \\
\text { al. (1998) }\end{array}$ \\
\hline Iridaceae & Libertia tricocca Phil. & Vulnerable & Ravenna et al. (1998) \\
\hline Lomariopsidaceae & Elaphoglossum fonkii (Phil.) T. Moore & Rara & $\begin{array}{c}\text { Rodríguez (1989), Baeza et } \\
\text { al. (1998) }\end{array}$ \\
\hline Lomariopsidaceae & Elaphoglossum gayanum (Fee) T. Moore & Vulnerable & Rodríguez (1989), \\
\hline Lycopodiaceae & Lycopodium paniculatum Desv. & Vulnerable & Baeza et al. (1998) \\
\hline
\end{tabular}


TABla VIII. Flora vascular de la Reserva Biológica de Huilo Huilo (Región de Los Ríos, Chile): comparación del índice de biodiversidad (Zuloaga et al. 1999) con otras áreas equivalentes del sur de Chile y Argentina.

TABLE VIII. Vascular flora at Huilo Huilo Reserve (Región de Los Ríos, Chile): biodiversity index (Zuloaga et al. 1999); a comparison with other areas from the same ecoregion.

\begin{tabular}{|c|c|c|c|c|c|}
\hline LOCALIDAD & REGIÓN-PÁIS & $\begin{array}{l}\text { NÚMERO DE } \\
\text { ESPECIES }\end{array}$ & TAMAÑO (HA) & $\begin{array}{c}\text { ÍNDICE DE } \\
\text { BIODIVERSIDAD }\end{array}$ & FuENTE \\
\hline MN Contulmo & VIII & 288 & 82 & 65,4 & Baeza et al. 1999 \\
\hline RN Tolhuaca & IX & 227 & 6.400 & 25,9 & Ramírez 1978 \\
\hline RN Malalcahuello & IX & 211 & 31.200 & 20,4 & Becerra \& Faúndez 1999 \\
\hline RB Huilo Huilo & XIV & 407 & 20.000 & 41 & En este trabajo \\
\hline PN Puyehue & $\mathrm{X}$ & 237 & 106.000 & 20,4 & Muñoz 1980 \\
\hline PN Laguna San Rafael & $\mathrm{XI}$ & 235 & 1.000 .000 & 17 & Teillier \& Marticorena 2002 \\
\hline Cuencas Baker y Pascua & XI & 342 & 21.600 & 27,8 & Rodríguez et al. 2008 \\
\hline PN Nahuel Huapi (estimado) & Argentina & 860 & 709.886 & 63,8 & Ferreyra et al. 1998 \\
\hline PN Copahue & Argentina & 365 & 28.000 & 35,6 & Gandullo et al. 2004 \\
\hline
\end{tabular}

ESPECTRO DE LAS FORMAS DE VIDA

El espectro de formas de vida, con dominancia de hemicriptófitos, corresponde con el esperado para ambientes templados (Braun-Blanquet 1979); donde también es esperada la presencia importante de los fanerófitos. En comparación con los datos presentados por Arroyo et al. (1995) respecto de la frecuencia de los diferentes tipos de formas de crecimiento o hábito de las especies en los bosques lluvioso-templados, se indica que el número de árboles y arbustos en Huilo Huilo alcanza a 72, puesto que se incluyen en el análisis tanto especies del bosque valdiviano (56 en Arroyo et al. 1996) como del nordpatagónico (48), particularmente del bosque de lenga; las leñosas trepadoras alcanzan a 13 , una riqueza similar a la del bosque valdiviano (14); finalmente los helechos reúnen a 41 especies, sobrepasando tanto al bosque valdiviano (30) como al nord-patagónico (39).

\section{Similitud ENTRE COMUNIDADES DE VEGETACiÓN}

Se efectuó un análisis de la similitud en la composición de especies de las comunidades analizadas, con base en el coeficiente de similitud de Jaccard, excluyendo a los humedales que presentan una composición de flora completamente particular. Los resultados de las comparaciones se muestran en la Figura 11. Se observa que, en general, el coeficiente de similitud entre comunidades es bajo, menor que un $50 \%$, ordenándose principalmente en un gradiente de temperaturas, donde los bosques más termófilos, el de tipo valdiviano (BV) y los montanos de Nothofagus dombeyi (coigüe-ND), se ubican muy cercanos en su composición, y algo distanciados de los demás bosques como el de Nothofagus pumilio (lenga-NL) y N. antarctica (ñirre-NA); este último se ubica entre el matorral subandino (SA) y la vegetación altoandina (AA), algo que debe explicarse por la singularidad de varios elementos de flora y por su ubicación especial en la pampa Pilmaiquén, donde, al parecer, a una baja altitud se registran condiciones de muy bajas temperaturas generando una inversión térmica que se refleja en que los bosques de $N$. antarctica nordpatagónicos y andinos se ubican más abajo que los de N.dombeyi (Figura 11); las comunidades menos similares son las praderas, de origen antropogénico, que albergan numerosas especies exóticas naturalizadas y muy pocas nativas.

Los bajos coeficientes de similitud dan cuenta en conclusión de una importante diversidad entre comunidades, lo que complementa la diversidad de especies, haciendo de la reserva un lugar importante para la conservación de la vegetación del bosque lluvioso templado austral. 
Flora vascular de la Reserva Biológica Huilo Huilo: TeILLIER, S. ET AL.

TABla IX. Flora vascular de la Reserva Biológica de Huilo Huilo (Región de Los Ríos, Chile): representatividad de la flora de la reserva en el marco de la ecorregión de los bosques templados australes (Fuente: Arroyo et al. 1995).

TABLE IX. Vascular flora at Huilo Huilo Reserve (Región de Los Ríos, Chile): level of representativity of the species from temperate austral forests at the reserve (Source: Arroyo et al. 1995).

\begin{tabular}{|c|c|c|c|}
\hline REPRESENTATIVIDAD POR TAXONES & $\begin{array}{c}\text { FLORA DEL BOSQUE TEMPLADO } \\
\text { AUSTRAL } \\
\mathrm{N}^{\circ} \text { DE ESPECIES }\end{array}$ & $\begin{array}{c}\text { FLORA DEL BOSQUE TEMPLADO } \\
\text { AUSTRAL EN LA RB HUILO } \\
\text { HUILO } \\
\mathrm{N}^{\circ} \text { DE ESPECIES }\end{array}$ & $\begin{array}{l}\text { PorCENTAJE DEL TOTAL DE ESPECIES } \\
\text { DEL BOSQUE TEMPLADO AUSTRAL } \\
\text { PRESENTES EN LA RB HUILO HuILO }\end{array}$ \\
\hline FAMILIAS & 96 & 79 & 82,3 \\
\hline Pteridophyta & 12 & 11 & 91,7 \\
\hline Pinophyta & 3 & 1 & 33,3 \\
\hline Magnoliophyta-Magnoliopsida & 68 & 57 & 83,8 \\
\hline Magnoliophyta-Liliopsida & 13 & 10 & 76,9 \\
\hline GÉNEROS & 205 & 152 & 77 \\
\hline Pteridophyta & 23 & 19 & 82,6 \\
\hline Pinophyta & 7 & 1 & 14,3 \\
\hline Magnoliophyta-Magnoliopsida & 133 & 101 & 75,9 \\
\hline Magnoliophyta-Liliopsida & 42 & 31 & 73,8 \\
\hline
\end{tabular}

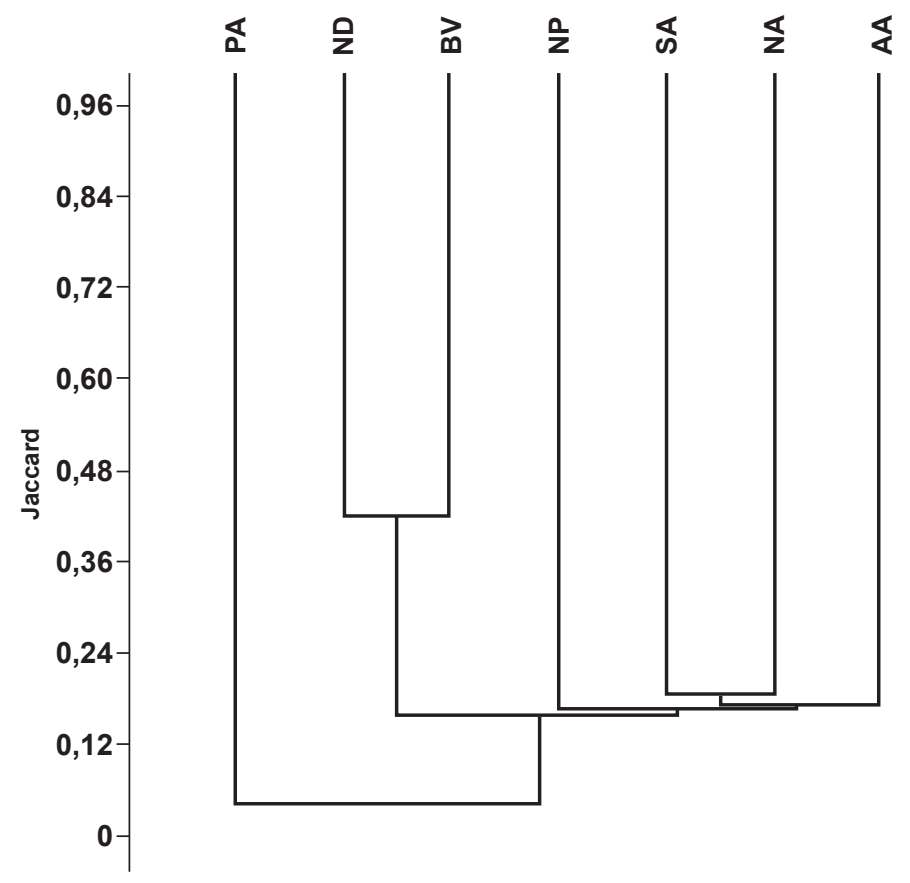

Figura 11. Flora vascular de la Reserva Biológica de Huilo Huilo (Región de Los Ríos, Chile): dendrograma de los resultados del análisis de similitud de comunidades utilizando el coeficiente de Jaccard. PA: praderas antropogénicas; ND: bosque de Nothofagus dombeyi; BV: bosque de tipo valdiviano; NP: bosque de Nothofagus pumilio; SA: matorral subandino; NA: bosque de Nothofagus antarctica; AA: matorral altoandino.

Figure 11. Vascular flora at Huilo Huilo Reserve (Región de Los Ríos, Chile): Jaccard index of similarity based dendrograme for plant type communities at the reserve. PA: antropogenic grassland; ND: Nothofagus dombeyi forest; BV: Valdivian type forest; NP: Nothofagus pumilio forest; SA: subandean scrub; NA: Nothofagus antarctica forest; AA: high Andean scrub. 
TABla X : Flora vascular de la Reserva Biológica de Huilo Huilo (Región de Los Ríos, Chile): géneros endémicos de la flora del bosque templado austral presentes en el área de estudio.

TABle X : Vascular flora at Huilo Huilo Reserve (Región de Los Ríos, Chile): endemics genera from temperate austral forests at the reserve.

\begin{tabular}{ll}
\hline FAMILIA & GÉNERO \\
\hline Aextoxicaceae* & Aextoxicon \\
Asteraceae & Acrisone \\
Bignoniaceae & Campsidium, Elytropus \\
Gesneriaceae & Asteranthera, Mitraria \\
Lardizabalaceae & Boquila \\
Hymenophyllaceae & Hymenoglossum \\
Monimiaceae & Laureliopsis \\
Misodendraceae & Misodendron \\
Myrtaceae & Amomyrtus, Luma \\
Philesiaceae & Lapageria \\
Podocarpaceae & Saxegothaea \\
Proteaceae & Embothrium \\
Santalaceae & Myoschilos \\
Scrophulariaceae & Fonkia \\
Thymelaceae & Ovidia \\
\hline
\end{tabular}

*Familia endémica / Endemic family.

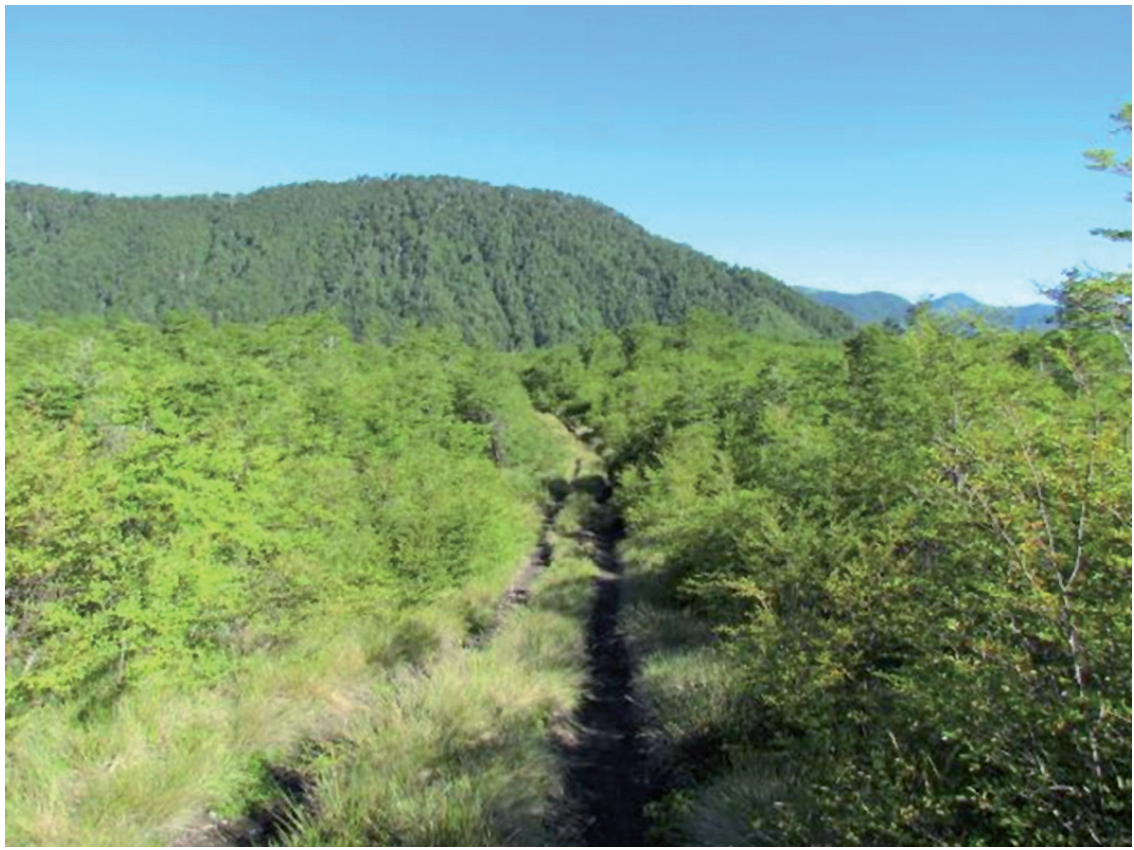

Figura 12. Pampa Pilmaiquén, bosque de Nothofagus antarctica en un sitio con probable inversión térmica. Al fondo se observa el bosque de $N$. dombeyi ubicado a mayor altitud en una posición "invertida" en relación a una gradiente normal donde $N$. antarctica forma el límite superior de la vegetación leñosa.

Figure 12. Pampa Pilmaiquén, Nothofagus antarctica forest at a probably termic inversion site. The $N$. dombeyi forest at the bottom appears at a higher site, an inverted position face to normal altitudinal gradient, where $N$. antarctica represent normally the tree line. 


\section{AGRADECIMIENTOS}

Los autores agradecen a la fundación Huilo Huilo por hacer posible este proyecto en términos logísticos y financieros. Por su valiosa ayuda en terreno se le agradece a Eduardo Arias y a Emilio y Robinson Sandoval.

\section{BIBLIOGRAFÍA}

Armesto, J.J., P. León-Lobos \& M.T.K. Arroyo. 1996. Los bosques templados del sur de Chile y Argentina: una isla biogeográfica. En: J. J. Armesto, C. Villagrán y M. K. Arroyo (eds.), Ecología de los Bosques Nativos de Chile, pp 23-28. Editorial Universitaria, Santiago, Chile.

Arroyo, M.T.K., L. Cavieres, A. Peñaloza, M. Riveros, A. Faggi. 1995. Relaciones fitogeográficas y patrones regionales de riqueza de especies en la flora del bosque lluvioso templado de Sudamérica. En: En: J. J. Armesto, C. Villagrán y M. K. Arroyo (eds.), Ecología de los Bosques Nativos de Chile, 71-99. Editorial Universitaria, Santiago, Chile.

Baeza, C. M., E. Barrera, J. Flores, C. Ramírez \& R. Rodríguez. 1998. Categorías de conservación de pteridophyta nativas de Chile. Boletín del Museo Nacional de Historia Natural 47: 23-46.

Baeza, C. M., C. Marticorena \& R. Rodríguez. 1999. Catálogo de la flora vascular del Monumento Natural Contulmo, Chile. Gayana Botánica 56(2): 125-135.

Becerra, P. \& L. Faúndez. 1999. Diversidad florística de la Reserva Nacional Malalcahuello, IX Región, Chile. Chloris Chilensis. Año 2, No1: http://www.chlorischile.cl (Consultada: 25 octubre 2012).

Becerra, P. \& G. Cruz. 2000. Diversidad vegetacional de la Reserva Nacional Malalcahuello, IX Región de Chile. Bosque 21(2): 47-68.

Benoit, I. 1989. Libro rojo de la flora terrestre de Chile. Corporación Nacional Forestal, Santiago, Chile.

Braun-Blanquet, J. 1979. Fitosociología. Bases para el estudio de las comunidades vegetales. H. Blume Ediciones. $820 \mathrm{pp}$.

Claude, M. 1997. Una vez más la miseria ¿Es Chile un país sustentable? LOM Ediciones Ltda. Santiago, Chile. 216 pp.

CONAMA. 2005. Política nacional de áreas protegidas. Documento on line en la URL: http://146.155.48.139/gestioncostera/ pdf/Chile/politica_areas_protegidas.pdf (Consultada, 8 -octubre-2012).

Correa, M.N. (ed.). 1984-1999. Flora Patagónica (República Argentina).Tomo VIII, partes I-VII). I.N.T.A. Argentina.

Di Castri, F. \& E. Hajek. 1976. Bioclimatología de Chile. Ediciones Universidad Católica de Chile. 163 pp.

Dinerstein, E., D. Olson, D. Graham, A. Webster, S. Primm, M. BoOKBINDER \& G. Ledec. 1995. A conservation Assessment of the Terrestrial Ecoregions of Latin America and the Caribbean. The International Bank for Reconstruction and Development / The World Bank, Washington D.C., US.

Farías, A., D. Tecklin \& P. Pliscoff. 2004. Análisis del avance hasta la fecha de la definición de las áreas prioritarias para la conservación de la biodiversidad en la Región de Los Lagos. Documento 8. Serie de publicaciones WWF-Chile. Ferreyra, M., S. Clayton \& C. Ezcurra. 1998. La flora altoandina de los sectores este y oeste del Parque Nacional Nahuel Huapi, Argentina. Darwiniana 36(1-4): 65-79.

FONT QUER, P. 1982. Diccionario de botánica. Editorial Labor.1244 pp.

Fundación Huilo Huilo. 2009. Memoria. 61pp.

Gajardo, R. 1994. La vegetación natural de Chile. Clasificación y distribución geográfica. Editorial Universitaria, Santiago. $164 \mathrm{pp}$.

Gandullo, R., E. Maletti \& A. M. Faggi. 2004. Diversidad florística del Parque Provincial Copahue, Neuquén, Argentina. Boletín de la Sociedad Argentina de Botánica. 39(3-4) 265-281.

Hechenleitner, V. P., M. F. Gardner, P. I. Thomas, C. Echeverría, B. Escobar, P. Brownless \& C. Martínez. 2005. Plantas amenazadas del centro-sur de Chile. Distribución, conservación y propagación. Primera edición. Universidad Austral de Chile y Jardín Botánico de Edinburgo. 187 pp.

Hoffmann, A. E. \& A. Flores. 1989. El estado de conservación de las plantas suculentas chilenas: una evaluación preliminar. En: I. Benoit (ed.), Libro rojo de la flora terrestre de Chile, pp. 111-127. Corporación Nacional Forestal, Chile.

Luebert, F. \& P. Pliscoff. 2005. Sobre los límites del bosque valdiviano. Chloris Chilensis. Año $8 \mathrm{~N}^{\mathrm{o}}$ 1. URL: http:// www.chlorischile.cl (Consultada 8 de octubre, 2012).

Macaya-Berti, J., S. Teillier \& A. Marticorena. 2012. Hallazgo del helecho Trichomanes exsectum Kunze en la precordillera de los Andes, Chile. Chloris Chilensis. Año15: $N^{\circ} 1$. URL://www.chlorischile.cl (Consultada 25 de octubre 2012).

Marticorena, C. 1990. Contribución a la estadística de la flora vascular de Chile. Gayana Botánica 47(3-4): 85-113.

Marticorena, C. \& M. QuezadA. 1985. Catálogo de la flora vascular de Chile. Gayana Botánica 42(1-2): 1-158.

Marticorena, C. \& R. Rodríguez. 1995. Flora de Chile. Vol I. Pteridophyta-Gymnospermae. Universidad de Concepción, Chile. 351 pp.

Marticorena, C. \& R. Rodríguez. 2001. Flora de Chile. Vol 2. Winteraceae-Ranunculaceae. 99 pp. Universidad de Concepción. Chile.

Marticorena, C. \& R. Rodríguez. 2003. Flora de Chile. Vol 2(2). Berberidaceae-Betulaceae. 93 pp. Universidad de Concepción. Chile.

Marticorena, C. \& R. Rodríguez. 2005. Flora de Chile. Vol 2(3). Plumbaginaceae-Malvaceae. 127 pp. Universidad de Concepción. Chile.

Marticorena, C. \& R. Rodríguez. 2011. Flora de Chile. Vol. 3(1). Misodendraceae-Zygophyllaceae. 148 pp. Universidad de Concepción. Chile.

Matthei, O. 1995. Manual de las malezas que crecen en Chile. Alfabeta Impresores, Santiago. 545 pp.

MuÑoz-Schick, M. 1980. Flora del Parque Nacional Puyehue. Editorial Universitaria. Santiago, Chile. 557 pp.

Ministerio Secretaría General de la Presidencia (MINSEGPRES). 2007. Decreto Supremo $\mathrm{N}^{\mathrm{o}} 151$, promulgado el 6 de diciembre de 2006; publicado en el Diario Oficial el 24 de marzo de 2007.

2008. Decreto Supremo No 50, promulgado el 24 de abril de 2008; publicado en el Diario Oficial el 30 de junio de 2008.

2008. Decreto Supremo No 51, promulgado el 24 de abril de 2008; publicado en el Diario Oficial el 30 de junio de 
2008.

- 2009. Decreto Supremo No 23, promulgado el 3 de marzo de 2009; publicado en el Diario Oficial el 7 de mayo de 2009 .

- 2011. Decreto Supremo $\mathrm{N}^{\mathrm{o}} 33$, promulgado el $7 \mathrm{de}$ septiembre de 2011; publicado en el Diario Oficial el 27 de febrero de 2012.

- 2011. Decreto Supremo No 41, promulgado el 30 de noviembre de 2011; publicado en el Diario Oficial el 11 de abril de 2012.

- 2011. Decreto Supremo No 42, promulgado el 30 de noviembre de 2011; publicado en el Diario Oficial el 11 de abril de 2012.

- 2012. Decreto Supremo N¹9, promulgado el 26 de junio de 2012; publicado el 11 de febrero de 2013.

Muñoz, M., H. NúÑEz \& J. YÁñEz. 1996. Libro rojo de los Sitios Prioritarios para la Conservación de la diversidad biológica en Chile. CONAF. 203 pp.

Myers, N., R.A. Mittermeier, C.G. Mittermeier, G.A.B. DA Fonseca \& J. Kent. 2000. Biodiversity hotspots for conservation priorities. Nature 403: 853-858.

Oberdorfer, E. 1960. Pflanzensoziologische Studien in Chile. Ein Vergleich mit Europa. Flora et Vegetatio Mundi 2: 1-208.

Ramírez, C. 1978. Estudio florístico y vegetacional del Parque Nacional Tolhuaca. Publicación ocasional del Museo Nacional de Historia Natural 24: 1-23.

Ravenna, P.F, S.Teillier, J. Macaya, R. Rodríguez \& O. Zöllner. 1998. Categorías de conservación de las plantas bulbosas nativas de Chile. Boletín del Museo nacional de Historia Natural: 47: 68.

Rodríguez, R. 1989. Pteridophyta de Chile continental amenazados de extinción. En: I. Benoit (ed.), Libro rojo de la flora terrestre de Chile, pp. 129-146. Corporación Nacional Forestal, Chile.

Rodríguez, R., A. Marticorena \& E. Teneb. 2008. Plantas vasculares de los ríos Baker y Pascua, Región de Aisén, Chile. Gayana Botánica 65(1): 39-70.

SCHMithüSEN, J. 1956. Die raunmliche Ordnung der chilenischen Vegetation. Bonner Geographische Abhandlungen 17:
$1-86$.

Sмiтн, C. 2001 (en adelante). Terrestrial Ecoregions. Temperate broadleaf and mixed forests. Southern South America: Chile and Argentina en el sitio web de la WWF. URL: $\quad \mathrm{http}: / /$ worldwildlife.org/ecoregions/nt0404 (Consultada 25/10/2012).

Stevens, P.F. (2001 en adelante). Angiosperm Phylogeny Website. Versión del 12 de julio $2012 \mathrm{http}: / /$ www.mobot.org/ MOBOT/research/APweb/ (Consultada 25/10/2012).

Teillier, S. \& C. Marticorena. 2002. Riqueza florística del Parque Nacional Laguna San Rafael, XI Región, Chile. Boletín del Museo Nacional de Historia Natural de Santiago 51: 43-73.

Tomé, A., S. Teillier \& R. Howarth. 2007. Contribución al conocimiento de la flora vascular de la Reserva Nacional Tamango, XI Región de Aisén, Chile. Boletín del Museo Nacional de Historia Natural de Santiago. Boletín del Museo Nacional de Historia Natural 56:9-25.

World Wildlife Foundation. 2001. A biodiversity vision for the Valdivian Temperate Rainforest Ecoregion. Washington, D.C.

World Wildlife Foundation. 2009. Protecting the Valdivian Forests of Chile and Argentina. http://wwf.panda.org/es/donde trabajamos/paises/chile/index.cfm? uProjectID $=9$ L0803 (Consultado 23-10-2012).

Zizka, G., M. Schmidt, K. Schulte, P. Novoa, R. Pinto \& K. KöNIG. 2009. Chilean Bromeliaceae: diversity, distribution and evaluation of conservation status. Biodiversity Conservation (2009) 18: 2449-2471.

Zuloaga, F. O., O. Morrone \& M. Belgrano (eds.). 2008. Catálogo de las plantas vasculares del Cono Sur. Monograph Systematic Botany, Missouri Botanical Garden 107. Missouri Botanical Garden Press. St. Louis, MO. USA. Vol. I. 983 pp. Versión on line en el sitio: http://www2. darwin.edu.ar/Proyectos/FloraArgentina/FA.asp

Zuloaga, F.O., O. Morrone \& D. Rodríguez. 1999. Análisis de la biodiversidad en plantas vasculares de la Argentina. Kurtziana 27: 17-167.

\section{Anexo 1 \\ CATÁLOGO DE LAS ESPECIES}

Las formas de vida son las de Raunkiaer (Braun-Blanquet 1979), han sido traducidas según el uso del diccionario de botánica de Font Quer (1982). Las cifras en números romanos corresponden a las regiones administrativas de Chile.

\section{PTERIDOPHYTA}

\section{Adiantaceae}

Adiantum chilense Kaulf.

palito negro Hemicriptófito en roseta. Nativa (IV-XII). Crece en los bosques de tipo valdiviano y de Nothofagus dombeyi. Exs. S. Teillier, J. Delaunoy \& C. Bonnemaison, 6621 (CONC); S. Teillier, J. Macaya, J. Delaunoy \& C. Bonnemaison, 7294 (CONC).

\section{Adiantum sulphureum Kaulf.}

Hemicriptófito en roseta. Endémica de Chile y Argentina (VIII-X). Crece en el bosque de tipo valdiviano, escasa. Exs. S. Teillier,
J. Delaunoy \& C. Bonnemaison, 6622 (CONC); S. Teillier, J. Macaya, J. Delaunoy \& C. Bonnemaison, 7293, 7302 (CONC).

\section{Aspleniaceae}

Asplenium dareoides A.N.Desv.

filu-lahuén

Hemicriptófito en roseta o epífito arborícola. Endémica de Chile y Argentina (IV-X). Crece en el bosque valdiviano y en el de Nothofagus dombeyi, sobre rocas húmedas, troncos muertos o como epífito. Exs. S. Teillier, J. Delaunoy \& C. Bonnemaison, 7202 (CONC); S. Teillier, J. Macaya, J. Delaunoy \& C. Bonnemaison, 7295 (CONC).

\section{Asplenium trilobum Cav.}

Epífito arborícola. Endémica de Chile y Argentina (VIII-XI). Crece en el bosque valdiviano, donde es escasísima. Clasificada como "vulnerable" por Baeza et al. (1998), por la reducción permanente de su hábitat. Exs. S. Teillier, J. Macaya, J. Delaunoy \& C. Bonnemaison, 7348 (CONC). 


\section{ATHYRIACEAE}

Cystopteris fragilis (L.) Bernh.

Hemicriptófito en roseta. Nativa, se encuentra en casi todo el país. Crece en sitios muy húmedos en el bosque de Nothofagus dombeyi y en el matorral subandino. Exs. S. Teillier, J. Delaunoy \& C. Bonnemaison, 6657, 6658 (CONC).

\section{BLECHNACEAE}

Blechnum chilense (Kaulf.) Mett.

costilla de vaca Hemicriptófito en roseta. Endémica de Chile y Argentina (IVXII). Crece en los bosques de tipo valdiviano y de Nothofagus dombeyi, donde es más frecuente en los claros y en los taludes de los caminos. Exs. S. Teillier, J. Delaunoy, J. Macaya \& C. Bonnemaison, 7416 (CONC).

Blechnum hastatum Kaulf. arriquilquil Hemicriptófito en roseta. Endémica de Chile y Argentina (IV-X). Crece en los bosques de tipo valdiviano y de Nothofagus dombeyi. Exs. S. Teillier, J. Delaunoy \& C. Bonnemaison, 6593, 7153 (CONC).

\section{Blechnum magellanicum (Desv.) Mett.}

Caméfito. Endémica de Chile y Argentina (VII-XII). Crece en los bosques de tipo valdiviano y de Nothofagus dombeyi. Exs. S. Teillier, J. Delaunoy, J. Macaya \& C. Bonnemaison, 7417 (CONC).

\section{Blechnum microphyllum (Goldm.) C.V.Morton}

Hemicriptófito en roseta. Endémica de Chile y Argentina (RM$\mathrm{X})$. Crece en el matorral subandino, tanto en el volcán MochoChoshuenco, como en el camino a Puerto Pirehueico. Exs. S. Teillier, J. Delaunoy \& C. Bonnemaison, 6592, 7358 (CONC); S. Teillier, J. Delaunoy, J. Macaya \& C. Bonnemaison, 7443 (CONC).

\section{Blechnum mochaenum Kunkel}

Hemicriptófito en roseta. Endémica de Chile y Argentina (VIIXII). Crece en el bosque de tipo valdiviano, donde es frecuente. Exs. S. Teillier, J. Delaunoy \& C. Bonnemaison, 6924, 6925, 6926, 7154 (CONC); S. Teillier, J. Delaunoy, J. Macaya \& C. Bonnemaison, 7418 (CONC).

Blechnum penna-marina (Poir.) Kuhn

pinque Hemicriptófito en roseta. Nativa, no endémica (IX-XII). Es frecuente en el bosque de Nothofagus antarctica; crece, además, en los de tipo valdiviano y de Nothofagus dombeyi (coigüe). Exs. S. Teillier, J. Delaunoy \& C. Bonnemaison, 6594 (CONC).

\section{Dennstaedtiaceae}

Hypolepis poeppigii (Kunze) R.A.Rodr. huilel-lahuén Hemicriptófito en roseta. Nativa, no endémica (IV y luego en VIIXII). Higrófila; crece sólo en el bosque de tipo valdiviano, donde se observó un solo ejemplar a la orilla de un estero. En la Región de los Ríos se la considera como "vulnerable" (Baeza et al. 1998) debido a la reducción permanente de su hábitat. No coleccionado.

DiCKSONIACEAE

Lophosoria quadripinnata (J.F.Gmel.) C.Chr.

ampe Hemicriptófito rizomatoso. Endémica de Chile y Argentina (VII$\mathrm{XI}$ ). Crece en los bosques de tipo valdiviano y de Nothofagus dombeyi. Se clasificó como "vulnerable" por la fuerte reducción de su hábitat al nivel nacional (Baeza et al. 1998); también influye el exceso de explotación pues su follaje es profusamente usado en arreglos florales. Exs. S. Teillier, J. Delaunoy, J. Macaya \& C. Bonnemaison, 7276, 7420 (CONC).

DRYOPTERIDACEAE

Megalastrum spectabile (Kaulf.) A.R.Sm. et R.C.Moran

pesebre

Hemicriptófito rizomatoso. Endémica de Chile y Argentina (IVXI). Crece en los bosques de tipo valdiviano y de Nothofagus dombeyi, donde es menos frecuente. Está clasificada en la categoría de "rara" por Baeza et al. (1998), por ser poco abundante en su área de distribución. Exs. S. Teillier, J. Delaunoy \& C. Bonnemaison, 7155,7505 (CONC).

\section{Polystichum andinum Phil.}

Hemicriptófito en roseta. Endémica de Chile y Argentina (RMXII). Es parte de la vegetación altoandina. Exs. S. Teillier, J. Delaunoy \& C. Bonnemaison, 6816, 6818 (CONC).

Polystichum chilense (Christ.) Diels pelomén-lahuén Hemicriptófito en roseta. Endémica de Chile y Argentina (VIIIXII). Crece en los bosques de tipo valdiviano y de Nothofagus dombeyi. Exs. S. Teillier, J. Delaunoy \& C. Bonnemaison, 6815-A, 7156 (CONC); S. Teillier, J. Delaunoy, J. Macaya \& C. Bonnemaison, 7327, 7343,7369 (CONC).

Polystichum plicatum (Poepp. ex Kunze) Hicken Hemicriptófito en roseta. Endémica de Chile y Argentina (IV-XII). Crece en los bosques de Nothofagus dombeyi y de Nothofagus pumilio (lenga), también en el matorral subandino. Exs. S. Teillier, J. Delaunoy \& C. Bonnemaison, 6812, 6814, 7158 (CONC).

Polystichum subintegerrimum (Hook. \& Arn.) R.A.Rodr. Hemicriptófito en roseta. Endémica de Chile (VIII-X). Crece en los bosque de tipo valdiviano y en los de Nothofagus dombeyi. Está clasificada en la categoría de "rara" por Baeza et al. (1998), por ser poco abundante en toda su área de distribución. Exs. S. Teillier, J. Delaunoy \& C. Bonnemaison, 6815, 6923 (CONC); S. Teillier, J. Delaunoy, J. Macaya \& C. Bonnemaison, 7328, 7356, 7357, 7368,7370 (CONC).

Rumohra adiantiformis (G.Forst.) Ching

pereq Hemicriptófito rizomatoso. Nativa, no endémica (IV-XII). Crece sólo en el bosque de tipo valdiviano, escasa. Exs. S. Teillier, J. Delaunoy \& C. Bonnemaison, 6858, 6859 (CONC).

\section{EQUISETACEAE}

Equisetum bogotense Kunth limpia-plata Hemicriptófito rizomatosa. Nativa (XIV-XI). Crece en sitios húmedos como las orillas de los esteros y de los ríos. Exs. S. Teillier, J. Delaunoy \& C. Bonnemaison, 6720, 7247 (CONC).

GLeicheniaceae

Gleichenia cryptocarpa Hook.

hierba loza Hemicriptófito en roseta. Endémica de Chile y Argentina (VIIIXII). Crece en el bosque de Nothofagus antarctica (ñirre), muy escasa. Zuloaga et al. (2008) lo citan como Sticherus cryptocarpus (Hook.) Ching. Exs. S. Teillier, J. Delaunoy \& C. Bonnemaison, 6690 (CONC). 
Gleichenia squamulosa (A.N.Desv.) Moore

yerba loza Hemicriptófito en roseta. Endémica de Chile y Argentina (VII-XI). Crece en los bosques de tipo valdiviano y en el de Nothofagus dombeyi, muy escasa. Zuloaga et al. (2008) lo citan como Sticherus squamulosus (Desv.) Nakai. Exs. S. Teillier, J. Delaunoy, J. Macaya \& C. Bonnemaison, 7301, 7447 (CONC).

\section{GRAMMITIDACEAE}

Grammitis magellanica Desv.

Epífito arborícola. Nativa no endémica (VIII-XII). Crece en el bosque de tipo valdiviano, muy escasa. Una de las poblaciones crecía en una caverna muy húmeda en el matorral subandino en la falda del Mocho-Choshuenco. Fue clasificada como "vulnerable" por Baeza et al. (1998) por la reducción de su hábitat. Exs. S. Teillier, J. Delaunoy \& C. Bonnemaison, 6687, 6688 (CONC); S. Teillier, A. Marticorena, J. Macaya \& C. Bonnemaison, 7440 (CONC).

\section{HYMENOPHYLLACEAE}

Hymenoglossum cruentum (Cav.) C.Presl helecho película Hemicriptófito rizomatoso, generalmente epífita. Endémica de Chile y Argentina (VIII-XII). Crece en el bosque de tipo valdiviano. Clasificada como "vulnerable" por Baeza et al. (1998) por la paulatina disminución de su hábitat. Como todas las especies de la familia, no prospera fuera del bosque. Exs. S. Teillier, J. Delaunoy \& C. Bonnemaison, 6767 (CONC).

Hymenophyllum caudiculatum Mart. pallante chilote Hemicriptófito rizomatoso, epífito o terrestre. Nativa no endémica (VIII-XII). Crece en el bosque de tipo valdiviano. Especie clasificada como "vulnerable" por pérdida de hábitat (Baeza et al. 1998). Como todas las especies de la familia, no crece fuera del bosque. Exs. S. Teillier, J. Delaunoy \& C. Bonnemaison, 6768, 6769, 6770, 6782 (CONC); S. Teillier, J. Delaunoy, J. Macaya \& C. Bonnemaison, 7360 (CONC).

Hymenophyllum darwinii Hook. f. ex Bosch

Hemicriptófito rizomatoso, saxícola. Endémica de Chile y Argentina (VII-XII). Crece en el bosque de tipo valdiviano, en rocas húmedas, muy escasa. Exs. S. Teillier, J. Delaunoy \& C. Bonnemaison, 6773 (CONC); S. Teillier, J. Delaunoy, J. Macaya \& C. Bonnemaison, 7361 (CONC).

Hymenophyllum dentatum Cav. shushu-lahuén Hemicriptófito rizomatoso, epífito o terrestre. Endémica de Chile y Argentina (VIII-XI). Crece en los bosques de tipo valdiviano y en los de Nothofagus dombeyi. La especie más frecuente de los Hymenophyllum en el área. Exs. S. Teillier, J. Delaunoy \& C. Bonnemaison, 6774, 7213 (CONC).

Hymenophyllum dicranotrichum (C.Presl) Hook. ex Sadeb. Hemicriptófito rizomatoso, generalmente epífito. Endémica de Chile (VIII-XI). Crece en el bosque de tipo valdiviano. Se clasificó como "vulnerable" por su carácter de especie endémica y por la pérdida sostenida de su hábitat (Baeza et al. 1998). Exs. S. Teillier, J. Delaunoy \& C. Bonnemaison, 6775, 6776 (CONC); S. Teillier, J. Delaunoy, J. Macaya \& C. Bonnemaison, 7363 (CONC).

Hymenophyllum ferrugineum Colla

Hemicriptófito rizomatoso, terrestre. Nativa no endémica (VII-X).
Crece en el bosque de tipo valdiviano. Exs. S. Teillier, J. Delaunoy \& C. Bonnemaison, 6777, 6778, 6779 (CONC); S. Teillier, J. Delaunoy, J. Macaya \& C. Bonnemaison, 7362 (CONC).

Hymenophyllum fuciforme Sw.

Hemicriptófito rizomatoso, terrestre. Endémica de Chile (VII-X). Crece sólo en bosque de tipo valdiviano, en sectores de mucha humedad y poca luz, escasa. Es la especie de mayor tamaño entre los Hymenophyllum en Chile. Exs. S. Teillier, J. Delaunoy \& C. Bonnemaison, 6780, 6781 (CONC); S. Teillier, J. Delaunoy, J. Macaya \& C. Bonnemaison, 7292 (CONC).

Hymenophyllum krauseanum Phil.

Hemicriptófito rizomatoso, generalmente epífita. Endémica de Chile y Argentina (VIII-XI). Crece en el bosque de tipo valdiviano. Exs. S. Teillier, J. Delaunoy \& C. Bonnemaison, 6783, 7218 (CONC); S. Teillier, J. Delaunoy, J. Macaya \& C. Bonnemaison, 7326, 7367 (CONC).

Hymenophyllum pectinatum Cav.

Hemicriptófito rizomatoso, epífita o terrestre. Endémica de Chile y Argentina (VII-XII). Crece en los bosques de tipo valdiviano y en los de Nothofagus dombeyi. Exs. S. Teillier, J. Delaunoy \& C. Bonnemaison, 6786, 7215,7216 (CONC).

Hymenophyllum peltatum (Poir.) Desv.

Hemicriptófito rizomatoso, epífito o terrestre. Nativa no endémica (IV-XII). Crece en el bosque de tipo valdiviano y en el matorral subandino, en sitios húmedos y poco luminosos. Exs. S. Teillier, J. Delaunoy \& C. Bonnemaison, 6787, 7217, 7218 (CONC); S. Teillier, J. Delaunoy, J. Macaya \& C. Bonnemaison, 7349, 7350, 7351 (CONC).

\section{Hymenophyllum plicatum Kaulf.}

Hemicriptófito rizomatoso, epífito o terrestre. Endémica de Chile y Argentina (VI-XII). Crece en el bosque de tipo valdiviano, poco frecuente. Exs. S. Teillier, J. Delaunoy \& C. Bonnemaison, 6788, 7352, 7365 (CONC).

\section{Hymenophyllum seselifolium C.Pres1}

Hemicriptófito rizomatoso, epífito o terrestre. Endémica de Chile y Argentina (VIII-XII). Crece en el bosque de tipo valdiviano, poco frecuente. Exs. S. Teillier, J. Delaunoy \& C. Bonnemaison, 6784, 6785 (CONC); S. Teillier, J. Delaunoy, J. Macaya \& C. Bonnemaison, 7353, 7218-A (CONC).

Hymenophyllum tortuosum Hook. \& Grev.

Hemicriptófito rizomatoso, generalmente epífito. Especie nativa, no endémica (VIII-XII). Crece en el bosque de tipo valdiviano, escasa. Se clasificó como "vulnerable" por su permanente pérdida de hábitat (Baeza et al. 1998). Exs. S. Teillier, J. Delaunoy \& C. Bonnemaison, 6789, 6790 (CONC); S. Teillier, J. Delaunoy, J. Macaya \& C. Bonnemaison, 7364 (CONC).

Hymenophyllum umbratile Diem \& J.S.Licht.

Hemicriptófito rizomatoso, epífito o terrestre. Endémica de Chile y Argentina (XIV-X). Crece en el bosque de tipo valdiviano, poco frecuente. Exs. S. Teillier, J. Delaunoy \& C. Bonnemaison, 7354 (CONC); S. Teillier, J. Delaunoy, J. Macaya \& C. Bonnemaison, 7366 (CONC). 
Serpyllopsis caespitosa (Gaudich.) C.Chr.

Hemicriptófito rizomatoso, epífito. Endémica de Chile y Argentina (XIV-XII). Crece en el bosque de tipo valdiviano, muy escasa. Baeza et al. (1998) señalan que podría estar amenazada, pero que no existían datos suficientes para incluirla en una categoría. Exs. S. Teillier, J. Delaunoy, J. Macaya \& C. Bonnemaison, 7298, 7359 (CONC).

\section{Trichomanes exsectum Kunze}

Hemicriptófito rizomatoso, terrestre. Endémica de Chile (VII-X). Crece en el bosque valdiviano, muy escasa. Está clasificada como "rara" por su escasez natural (Rodríguez, 1989 y Baeza et al. 1998). Primera vez que se la describe para la vertiente occidental de la cordillera de los Andes (Macaya et al. 2012). Exs. S. Teillier, J. Delaunoy, J. Macaya \& C. Bonnemaison, 7300 (CONC).

\section{LOMARIOPSIDAE}

\section{Elaphoglossum fonkii (Phil.) T.Moore}

Hemicriptófito en roseta, saxícola obligado. Endémica de Chile (XIV-X). Crece en el bosque valdiviano, en rocas húmedas, muy escasa. Se clasificó como "rara" por su escasez natural (Rodríguez 1989, Baeza et al. 1998). Exs. S. Teillier, J. Delaunoy \& C. Bonnemaison, 6733 (CONC); S. Teillier, J. Delaunoy, J. Macaya \& C. Bonnemaison, 7305 (CONC).

\section{Elaphoglossum gayanum (Fée) T.Moore}

Hemicriptófito en roseta, saxícola obligado. Especie nativa, no endémica (IX-X). Crece en el bosque de tipo valdiviano, sobre una roca vertical, muy húmeda, escasísima; se observó un sólo ejemplar en el sendero del río Truful. Se clasificó como "vulnerable" por su escasez natural (Rodríguez 1989). Exs. S. Teillier, J. Delaunoy, J. Macaya \& C. Bonnemaison, 7305-A (CONC).

LYCOPODIACEAE

\section{Lycopodium alboffii Rolleri}

Hemicriptófito escapífero. Endémica de Chile y Argentina (XIVXII), nuevo límite de distribución norte de la especie. Crece en los claros del matorral subandino, sobre $1200 \mathrm{~m}$ de altitud. Exs. S. Teillier, J. Delaunoy \& C. Bonnemaison, 6760 (CONC).

Lycopodium magellanicum (P.Beauv.) Sw. pimpinela Hemicriptófito escapífero. Nativa, no endémica (VIII-XII). Crece en el bosque de Nothofagus pumilio y en los claros del matorral subandino. Exs. S. Teillier, J. Delaunoy \& C. Bonnemaison, 6761, 6762, 6763 (CONC).

Lycopodium paniculatum Desv.

palmita Hemicriptófito escapífero. Endémica de Chile y Argentina (VIIIXII). Crece en el bosque de tipo valdiviano. Clasificada como "vulnerable" por Baeza et al. (1998), por la extracción comercial intensiva de sus ramas para arreglos florales. Exs. S. Teillier, J. Delaunoy \& C. Bonnemaison, 6764 (CONC); S. Teillier, J. Delaunoy, J. Macaya \& C. Bonnemaison, 7419 (CONC).

\section{PolyPodiaceae}

\section{Polypodium feuillei Bertero}

calaguala, pilla vilcún Hemicriptófito rizomatoso, epífito. Endémica de Chile y Argentina (IV-XII). Crece en los bosques de tipo valdiviano y de Nothofagus dombeyi. Zuloaga et al. (2008) lo citan como Synammia feuillei (Bertero) Copel. Exs. S. Teillier, J. Delaunoy \& C. Bonnemaison, 6819 (CONC).

\section{PINOPHYTA (GIMNOSPERMAS)}

\section{Pinaceae}

Pinus contorta Douglas \& Loudon

pino Megafanerófito. Especie de origen norteamericano, asilvestrada en Chile y Argentina. Crece en la pampa Pilmaiquén, donde está invadiendo a partir de plantaciones de los años 1960. Invasiones por esta especie han sido reportadas en Nueva Zelanda, Europa, Argentina y varios parques y reservas protegidas del sur de Chile. Exs. S. Teillier, J. Delaunoy \& C. Bonnemaison 7281 (CONC).

Pseudotsuga menziesii (Mirb.) Franco pino de Oregón Megafanerófito. Especie de origen norteamericano, asilvestrada en Chile. Crece en el valle del río Pillanleufú, siendo la fuente plantaciones hechas en algunos de los cerros aledaños para controlar la erosión. Especie con gran potencial invasor debido a la facilidad con que germinan sus semillas. Actualmente se intenta controlar su avance en el Parque Nacional Conguillío y en la Reserva Nacional Malalcahuello.

\section{PoDocarpaceae}

Saxegothaea conspicua Lindley

mañío hembra Megafanerófito. Endémica de Chile y Argentina (VII-X). Crece en el bosque de tipo valdiviano y en el de Nothofagus dombeyi (coigüe). Hechenleitner et al. (2005), la clasifican como "casi amenazada" por su permanente corta para obtener madera o leña, además de los cambios de uso de suelo, frecuentes en su área de distribución para sustituirla por árboles exóticos. Exs. S. Teillier, J. Delaunoy \& C. Bonnemaison 6855, 6856 (CONC).

\section{ESPECIES CULTIVADAS}

En el área de este estudio se encuentran algunas plantaciones de Thuja plicata (Cupressaceae) y de Araucaria araucana (Araucariaceae).

\section{MAGNOLIOPHYTA (ANGIOSPERMAS) \\ DICOTYLEDONATAE S.L. (MAGNOLIOPSIDA )}

Adoxaceae

Sambucus nigra L.

sauco Megafanerófito. Alóctona asilvestrada (VIII-XII); originaria de Eurasia. Crecen en el bosque de tipo valdiviano aledaño al poblado de Neltume. No coleccionado.

\section{AeXtoxicaceae}

Aextoxicon punctatum Ruiz \& Pav. olivillo Megafanerófito. Endémica de Chile y Argentina (IV-X). Uno de los árboles dominantes en el bosque de tipo valdiviano. Exs. S. Teillier, J. Delaunoy. J. Macaya \& C. Bonnemaison 7310 (CONC).

ApIaceae (Umbelliferae)

Azorella lycopodioides Gaudich.

llareta Caméfito, subarbusto. Endémica de Chile y Argentina (VI-XII). Forma parte de la vegetación altoandina, escasa. Exs. S. Teillier, J. Delaunoy, J. Macaya \& C. Bonnemaison 7388 (CONC).

Azorella trifoliolata Clos

llareta

Hemicriptófito en roseta. Endémica de Chile y Argentina (III-XII). Es parte de la vegetación higrófila de la laguna Trarolafquén. Exs. 
S. Teillier, J. Delaunoy \& C. Bonnemaison 6606, 6607 (CONC).

Daucus carota L.

zanahoria

Hemicriptófito bienal. Alóctona asilvestrada (V-X), originaria de Eurasia. Crece en las praderas con las gramíneas Anthoxanthum odoratum y Holcus lanatus. Exs. S. Teillier, J. Delaunoy \& C. Bonnemaison 6655 (CONC).

Hydrocotyle chamaemorus Cham. \& Schltdl. malva del monte Hemicriptófito repente. Endémica de Chile y Argentina (VIII-X). Crece en sitios húmedos. Exs. S. Teillier, J. Delaunoy \& C. Bonnemaison 6887 (CONC); S. Teillier, J. Macaya, J. Delaunoy \& C. Bonnemaison 7235 (CONC).

\section{Hydrocotyle indecora DC.}

Hemicriptófito repente. Endémica de Chile y Argentina (IV-XI). Crece en sitios húmedos, algo alterados; poco frecuente. Exs. S. Teillier, J. Delaunoy \& C. Bonnemaison 6884 (CONC); S. Teillier, J. Macaya, J. Delaunoy \& C. Bonnemaison 7404 (CONC).

Osmorhiza chilensis Hook. \& Arn. asta de cabra Hemicriptófito escapífero. Nativa no endémica (IV-XII). Crece en todos los tipos de bosque. Exs. S. Teillier, J. Delaunoy \& C. Bonnemaison 6835,7160 (CONC).

Apocynaceae

Diplolepis pachyphylla (Decne.) Hechem \& C.Ezcurra. voquicillo Fanerófito trepador. Endémica de Chile y Argentina (VII-X). Crece sólo en el bosque de tipo valdiviano, muy escasa. No coleccionada.

Elytropus chilensis (A. DC.) Müll. Arg. quilmai Fanerófito trepador. Endémica de Chile y Argentina (VII-XI). Crece sólo en el bosque de tipo valdiviano. Exs. S. Teillier, J. Delaunoy \& C. Bonnemaison 6732, 7190 (CONC); S. Teillier, J. Macaya, J. Delaunoy \& C. Bonnemaison 7315 (CONC).

\section{Araliaceae}

Pseudopanax laetevirens (Gay) Franchet sauco cimarrón Megafanerófito, frecuente como epífito arborícola. Endémica de Chile y Argentina (VII-XII). Crece en los bosques de tipo valdiviano y en los de Nothofagus dombeyi. Exs. S. Teillier, J. Delaunoy \& C. Bonnemaison 6869 (CONC).

\section{Asteraceae (Compositae)}

Achillea millefolium $\mathrm{L}$.

milenrama Hemicriptófito escapífero. Alóctona asilvestrada (RM-XII). Crece en las praderas antropizadas, muy frecuente. Exs. S. Teillier, J. Delaunoy \& C. Bonnemaison 6631 (CONC).

Acrisione cymosa (J.Remy) B.Nord. palpalén Megafanerófito. Endémica de Chile (XIV-XI). Crece en el bosque de Nothofagus dombeyi, escasa. Exs. S. Teillier, J. Delaunoy \& C. Bonnemaison 6630 (CONC).

Adenocaulon chilense Less.

Hemicriptófito en roseta. Endémica de Chile y Argentina (VIIIXII). Crece en casi todos los tipos de bosque. Exs. S. Teillier, J. Delaunoy \& C. Bonnemaison 6623, 7237 (CONC).
Anthemis cotula L.

manzanillón Terófito. Especie alóctona asilvestrada (XIV-XII), proveniente de Eurasia. Crece sólo en praderas fuertemente antropizadas (nitrófila). No coleccionada.

\section{Arnica angustifolia Vahl}

Hemicriptófito escapífero. Alóctona, asilvestrada en la cordillera de Valdivia, desde al menos 1897, originaria del hemisferio norte. Crece en la parte más alta del camino a Puerto Pirehueico, ruta antiguamente utilizada para cruzar a Argentina, donde también se encuentra naturalizada en los alrededores de San Martín de los Andes. Exs. S. Teillier, J. Delaunoy \& C. Bonnemaison 6611 (CONC).

\section{Baccharis elaeoides J.Remy}

Nanofanerófito. Endémica de Chile (XIV-X). Crece en el bosque de Nothofagus dombeyi, poco frecuente. En el área forma híbridos con Baccharis patagonica ssp. palenae. Exs. S. Teillier, J. Delaunoy \& C. Bonnemaison 7268, 7547 (CONC).

Baccharis magellanica (Lam.) Pers. chilca Caméfito en espaldera. Endémica de Chile y Argentina (VII-XII). Crece en los claros del bosque de Nothofagus antarctica. Exs. S. Teillier, J. Macaya, J. Delaunoy \& C. Bonnemaison 7222, 7223 (CONC).

Baccharis nivalis (Wedd.) Sch. Bip. ex Phil.

Caméfito subarbusto. Endémica de Chile y Argentina (VIII-XII). Crece en el matorral subandino y en el bosque de Nothofagus pumilio. Es la menor de las especies del género y es muy poco leñosa. Exs. S. Teillier, J. Delaunoy \& C. Bonnemaison 6603, 6604 (CONC).

Baccharis obovata Hook. \& Arn.

Nanofanerófito. Endémica de Chile y Argentina (VIII-XI). Crece en el matorral subandino y en los bosques de Nothofagus dombeyi y Nothofagus pumilio. Exs. S. Teillier, J. Delaunoy \& C. Bonnemaison 6600, 6601, 6602 (CONC); S. Teillier, J. Macaya, J. Delaunoy \& C. Bonnemaison 7449 (CONC).

Baccharis patagonica Hook. \& Arn.

Nanofanerófito. Endémica de Chile y Argentina (VII-XII). Crece en los claros del bosque de Nothofagus antarctica. Exs. S. Teillier, J. Delaunoy \& C. Bonnemaison 7200-A, 7200 B (CONC); S. Teillier, J. Macaya, J. Delaunoy \& C. Bonnemaison 7269 (CONC).

Baccharis racemosa (Ruiz \& Pav.) DC. chilca Nanofanerófito. Endémica de Chile y Argentina (V-X). Crece en los claros de los bosques de tipo valdiviano y de los de Nothofagus dombeyi. Exs. S. Teillier, J. Delaunoy \& C. Bonnemaison 6599 (CONC);

Baccharis salicifolia (Ruiz \& Pav.) Pers.

Nanofanerófito. Especie nativa no endémica (XIV-X). Crece en sitios arenosos en el margen de los ríos Fuy y Pillanleufú y en el lago Pirehueico. Exs. S. Teillier, J. Macaya, J. Delaunoy \& C. Bonnemaison 7283 (CONC).

Centaurea nigra $\mathrm{L}$.

Hemicriptófito escapífero. Alóctona, asilvestrada (IX-XIV), 
originaria de Europa templada. Se encontró sólo en una pradera del valle del río Pillanleufú. Exs. S. Teillier, A. Marticorena, J. Macaya \& C. Bonnemaison 7374 (CONC).

Chiliotrichum diffusum (G.Forst.) Kuntze romerillo Nanofanerófito. Endémica de Chile y Argentina (VII-XII). Crece en el matorral subandino, escasa. Incluye a las poblaciones incluidas antes en Chiliotrichum rosmarinifolium. Exs. S. Teillier, J. Delaunoy \& C. Bonnemaison 6668, 7541 (CONC).

Cichorium intybus L.

achicoria

Hemicriptófito en roseta. Alóctona asilvestrada (III-XII), originaria de Eurasia. Escasa, crece sólo en las praderas muy alteradas. Exs. S. Teillier, J. Delaunoy, J. Macaya \& C. Bonnemaison 7342 (CONC).

Cirsium vulgare (Savi) Ten.

cardo Geófito con raíces gemíferas. Alóctona asilvestrada (IV-XII), originaria de Eurasia. Crece en praderas en sitios alterados. Exs. S. Teillier, J. Delaunoy, J. Macaya \& C. Bonnemaison 7288 (CONC).

\section{Conyza larrainiana J.Remy}

Hemicriptófito escapífero. Endémica de Chile y Argentina (XIVXII). Crece en los bosques de tipo valdiviano y de Nothofagus dombeyi. Exs. S. Teillier, J. Delaunoy \& C. Bonnemaison 7398 (CONC).

Conyza sumatrensis (Retz.) E.Walker var. leiotheca (S.F.Blake) Pruski \& G. Sancho

Hemicriptófito escapífero. Nativa no endémica (III-X). Crece en los bosques de tipo valdiviano y de Nothofagus dombeyi, en sitios alterados. Exs. S. Teillier, J. Delaunoy \& C. Bonnemaison 6663, 6664, 6665 (CONC); S. Teillier, J. Macaya, J. Delaunoy \& C. Bonnemaison 7488 (CONC); S. Teillier, A. Marticorena, J. Macaya \& C. Bonnemaison 7434 (CONC).

Crepis capillaris (L.) Wallr.

Hemicriptófito escapífero. Alóctona asilvestrada (RM-XII), originaria de Eurasia. Crece en las praderas y en los claros del bosque de tipo valdiviano donde es poco frecuente. Exs. S. Teillier, J. Delaunoy \& C. Bonnemaison 6660, 6661 (CONC).

Dasyphyllum diacanthoides (Less.) Cabrera trevo Megafanerófito. Endémica de Chile y Argentina (VII-XII). Crece en los bosques de tipo valdiviano y de Nothofagus dombeyi. Exs. S. Teillier, J. Macaya, J. Delaunoy \& C. Bonnemaison 7335 (CONC).

\section{Erigeron cinereus Hook. \& Arn.}

Hemicriptófito en roseta. Endémica de Chile y Argentina (VIIIXII). Forma parte de la vegetación altoandina, escasa. Exs. S. Teillier, J. Delaunoy \& C. Bonnemaison 6313, 6718, 7524, 7539 (CONC). S. Teillier, J. Macaya, A. Marticorena \& C. Bonnemaison 7409 (CONC).

Erigeron gilliesii (Hook. \& Arn.) Cabrera

Hemicriptófito en roseta. Endémica de Chile y Argentina (V-XII). Se encontró en el matorral subandino. Exs. S. Teillier, J. Macaya, A. Marticorena \& C. Bonnemaison 7410 (CONC).
Gamochaeta americana (Mill.) Wedd.

Hemicriptófito escapífero. Nativa no endémica (V-XII). Crece en las praderas antrópicas y en los sitios abiertos de los bosques de tipo valdiviano y de Nothofagus dombeyi. Exs. S. Teillier, J. Delaunoy \& C. Bonnemaison 6893, 6894 (CONC); S. Teillier, J. Macaya, J. Delaunoy \& C. Bonnemaison 7321 (CONC).

\section{Gamochaeta chamissonis (DC.) Cabrera}

Hemicriptófito escapífero. Endémica de Chile y Argentina (V-XIV). Crece en el bosque de Nothofagus dombeyi, muy escasa. Exs. S. Teillier, J. Delaunoy \& C. Bonnemaison 6890 (CONC).

Gamochaeta depilata (Phil.) Cabrera

Hemicriptófito en roseta. Endémica de Chile y Argentina (VIIXIV). Crece en el bosque de Nothofagus pumilio y en el matorral subandino, poco frecuente. Exs. S. Teillier, J. Delaunoy \& C. Bonnemaison 6892 (CONC); S. Teillier, J. Macaya, A. Marticorena \& C. Bonnemaison 7387 (CONC).

\section{Gamochaeta nivalis Cabrera}

Hemicriptófito en roseta. Endémica de Chile y Argentina (VII-X). Forma parte de la vegetación altoandina, poco frecuente. Exs. S. Teillier, J. Delaunoy \& C. Bonnemaison 6889, 6891 (CONC); S. Teillier, J. Macaya, A. Marticorena \& C. Bonnemaison 7438 (CONC).

Gamochaeta spiciformis (Sch. Bip.) Cabrera Hemicriptófito escapífero. Endémica de Chile y Argentina (VIIXII). Crece en los claros del bosque de tipo valdiviano y de Nothofagus dombeyi (coigüe). Exs. S. Teillier, J. Delaunoy \& C. Bonnemaison 6886, 6887, 6888 (CONC); S. Teillier, J. Macaya, J. Delaunoy \& C. Bonnemaison 7489 (CONC).

\section{Gnaphalium pratense Phil.}

Hemicriptófito escapífero. Endémica de Chile y Argentina (XIVXI). Crece en el bosque de Nothofagus dombeyi, muy escasa. Exs. S. Teillier, J. Delaunoy \& C. Bonnemaison 6889 (CONC).

Haplopappus glutinosus Cass.

Caméfito subarbusto. Endémica de Chile y Argentina (V-XI). Crece en las rocas húmedas aledañas a los ríos Fuy y Truful, poco frecuente. Exs. S. Teillier, J. Delaunoy \& C. Bonnemaison 6735, 7184 (CONC).

\section{Hieracium chilense Less.}

Hemicriptófito en roseta. Endémica de Chile y Argentina (VIIXII). Crece en el bosque de tipo valdiviano, muy escasa. Exs. S. Teillier, J. Delaunoy \& C. Bonnemaison 6736 (CONC).

Hypochaeris palustris (Phil.) De Wild.

Hemicriptófito en roseta. Endémica de Chile y Argentina (VIIIXII). Crece en el matorral subandino, en sitios húmedos. Exs. S. Teillier, J. Delaunoy \& C. Bonnemaison 6740, 6743, 6744, 7385, 7391, 7392 (CONC). S. Teillier, J. Macaya, J. Delaunoy \& C. Bonnemaison 7255, 7439 (CONC).

Hypochaeris radicata $\mathrm{L}$.

hierba del chancho Hemicriptófito en roseta. Alóctona asilvestrada (V-XII), originaria de Eurasia. Crece en las praderas, en los claros de los bosques y en las orillas de los caminos y senderos, indicador de alteración 
antrópica. Exs. S. Teillier, J. Delaunoy \& C. Bonnemaison 6741, 6742 (CONC).

Lagenophora hariotii Franch.

Hemicriptófito en roseta. Endémica de Chile y Argentina (VIIXII). Crece en el matorral subandino, escasa. Exs. S. Teillier, J. Macaya, J. Delaunoy \& C. Bonnemaison 7386 (CONC); S. Teillier, J. Macaya, A. Marticorena \& C. Bonnemaison 7444 (CONC).

\section{Lapsana communis L.}

Hemicriptófito en roseta. Alóctona asilvestrada (IV-XII), originaria de Eurasia. Crece en las orillas de los caminos y en los claros de los bosques. Exs. S. Teillier, J. Delaunoy \& C. Bonnemaison 6750 (CONC).

\section{Leontodon taraxacoides (Vill.) Mérat}

Hemicriptófito en roseta. Alóctona asilvestrada (V-X), originaria de Eurasia. Crece en sitios alterados, como orillas de los caminos y en las praderas sobrepastoreadas. Exs. S. Teillier, J. Delaunoy \& C. Bonnemaison 6751 (CONC).

Leucanthemum vulgare Lam. margarita Hemicriptófito escapífero. Alóctona asilvestrada (IV-XII), originaria de Eurasia. Crece en sitios alterados, como las orillas de los caminos y las praderas en exceso pastoreadas, donde es una de las especies dominantes. Exs. S. Teillier, J. Macaya, J. Delaunoy \& C. Bonnemaison 7258 (CONC).

Leucheria glacialis (Poepp. ex Less.) Reiche

Hemicriptófito escapífero. Endémica de Chile y Argentina (VIIXI). Se observó sólo en el río Pillanleufú, muy escasa. Exs. S. Teillier, J. Delaunoy \& C. Bonnemaison 6882 (CONC).

\section{Leucheria thermarum (Phil.) Reiche}

Hemicriptófito escapífero. Endémica de Chile y Argentina (VIII-XI). Crece en los bosques de Nothofagus pumilio y en el matorral subandino, escasa. Exs. S. Teillier, J. Delaunoy \& C. Bonnemaison 7533 (CONC); S. Teillier, J. Delaunoy, J. Macaya \& C. Bonnemaison 7260 .

\section{Lucilia araucana Phil.}

Hemicriptófito en roseta. Endémica de Chile y Argentina (VIII-X). Crece en los claros del matorral subandino, poco frecuente. Exs. S. Teillier, J. Delaunoy \& C. Bonnemaison 6757 (CONC).

Macrachaenium gracile Hook. $\mathrm{f}$.

Hemicriptófito escapífero. Endémica de Chile y Argentina (VIIIXII). Crece como sotobosque en los bosques de Nothofagus pumilio (lenga). Exs. S. Teillier, J. Delaunoy \& C. Bonnemaison 6806, 6807 (CONC).

\section{Madia sativa Molina}

melosa Terófito. Endémica de Chile y Argentina (III-XII), aunque se ha asilvestrado en otros países del mundo. Crece en sitios alterados, normalmente praderas de pastoreo o en sitios donde hubo faenas de aserradero, poco frecuente. Exs. S. Teillier, J. Delaunoy \& C. Bonnemaison 6804, 6805 (CONC).

Mutisia spinosa Ruiz \& Pav. clavel del campo Fanerófito, trepador. Endémica de Chile y Argentina (VII-XI).
Crece en el bosque de Nothofagus antarctica. Exs. S. Teillier, J. Delaunoy \& C. Bonnemaison 6786 (CONC).

Nassauvia dentata Griseb.

Caméfito subarbusto. Endémica de Chile y Argentina (VIII-XII). Forma parte de la vegetación altoandina, escasa. Exs. S. Teillier, J. Delaunoy \& C. Bonnemaison 6778, 6779 (CONC).

Nassauvia lagascae (D.Don) F. Meigen var. lanata (Phil.) Skottsb. Caméfito subarbusto. Variedad endémica de Chile y Argentina (VIIXII). Forma parte de la vegetación altoandina del volcán MochoChoshuenco. Exs. S. Teillier, J. Delaunoy \& C. Bonnemaison 6776, 6777 (CONC).

Nassauvia revoluta D.Don

cadislao

Caméfito subarbusto. Endémica de Chile y Argentina (VI-XII). Forma parte de la vegetación altoandina del volcán MochoChoshuenco. Exs. S. Teillier, J. Delaunoy \& C. Bonnemaison 6774, 6775 (CONC).

\section{Perezia pedicularidifolia Less.}

Hemicriptófito escapífero. Endémica de Chile y Argentina (VIIXI). Crece en matorral subandino. Exs. S. Teillier, J. Delaunoy \& C. Bonnemaison 6826, 6827 (CONC).

Perezia prenanthoides Less.

Hemicriptófito escapífero. Endémica de Chile y Argentina (VIII-X). Crece en el bosque de Nothofagus pumilio, poco frecuente. Exs. S. Teillier, J. Delaunoy \& C. Bonnemaison 6877, 6878 (CONC).

Senecio acanthifolius Hombr. \& Jacquinot

Hemicriptófito escapífero. Endémica de Chile y Argentina (XIVXII). Crece en el matorral subandino y en el bosque de Nothofagus pumilio, escasa. S. Teillier, J. Delaunoy \& C. Bonnemaison 6854 (CONC).

Senecio bipontinii Wedd.

Caméfito subarbusto. Endémica de Chile y Argentina (VIII-XIV). Una de las especies dominantes en la vegetación altoandina. Exs. S. Teillier, J. Delaunoy \& C. Bonnemaison 6853 (CONC); S. Teillier, J. Macaya, A. Marticorena \& C. Bonnemaison 7437 (CONC).

Senecio chilensis Less.

Caméfito subarbusto. Endémica de Chile y Argentina (V-X). Crece en los claros del bosque de Nothofagus antarctica y de Nothofagus dombeyi. Exs. S. Teillier, J. Delaunoy \& C. Bonnemaison 6851, 6852 (CONC); S. Teillier, J. Macaya, J. Delaunoy \& C. Bonnemaison 7296 (CONC).

Senecio chionophilus Phil.

Caméfito subarbusto. Endémica de Chile y Argentina (VIII-X). Crece en los claros del bosque de Nothofagus pumilio y en el matorral subandino, donde es una de las más frecuentes. Exs. S. Teillier, J. Delaunoy \& C. Bonnemaison 6848, 6849, 6850 (CONC).

Senecio hieracium J.Remy

Hemicriptófito escapífero. Endémica de Chile y Argentina (VII$\mathrm{XI})$. Crece en las praderas húmedas de altura, escasa. Exs. S. 
Teillier, J. Delaunoy \& C. Bonnemaison 6847 (CONC); S. Teillier, J. Macaya, A. Marticorena \& C. Bonnemaison 7395 (CONC).

Senecio otites Kunze ex DC.

trompetilla Nanofanerófito. Endémica de Chile y Argentina (VII-XI). Crece en los claros del bosque de Nothofagus dombeyi, en los taludes que dan al río Pillanleufú. Exs. S. Teillier, J. Macaya, A. Marticorena \& C. Bonnemaison 7373 (CONC).

\section{Senecio prenanthifolius Phil.}

Hemicriptófito escapífero. Endémica de Chile y Argentina (IX$\mathrm{X}$ ). Crece en los claros del bosque de Nothofagus pumilio, poco frecuente. Exs. S. Teillier, J. Delaunoy \& C. Bonnemaison 6845 (CONC).

Senecio trifurcatus (G.Forst.) Less.

Hemicriptófito escapífero. Endémica de Chile y Argentina (IXXII). Crece en el matorral subandino, poco frecuente. Exs. S. Teillier, J. Delaunoy \& C. Bonnemaison 6844 (CONC).

\section{Senecio triodon Phil.}

Caméfito subarbusto. Endémica de Chile y Argentina (VII-XII). Crece en el matorral subandino, poco frecuente. Exs. S. Teillier, J. Delaunoy \& C. Bonnemaison 6843 (CONC).

\section{Solidago chilensis Meyen}

Hemicriptófito escapífero. Especie nativa no endémica (XV-XI). Crece en praderas y claros del bosque de Nothofagus antarctica, poco frecuente. Exs. S. Teillier, J. Delaunoy \& C. Bonnemaison 6838 (CONC).

Sonchus asper (L.) Hill ñilhue Terófito. Alóctona, asilvestrada en casi todo el país. Crece en sitios alterados, en taludes de caminos y en praderas. Exs. S. Teillier, J. Macaya, A. Marticorena \& C. Bonnemaison 7274 (CONC).

\section{Symphyotrichum glabrifolium (DC.) G.L.Nesom}

Hemicriptófito en roseta. Endémica de Chile y Argentina (VIIXII). Crece en los claros del matorral subandino. Exs. S. Teillier, J. Delaunoy \& C. Bonnemaison 6614 (CONC).

\section{Taraxacum officinale Weber}

diente de león Hemicriptófito en roseta. Alóctona; en el país se ha naturalizado en prácticamente todas las regiones. No se coleccionó.

\section{ATHEROSPERMATACEAE}

Laureliopsis philippiana (Looser) Schodde

Megafanerófito. Endémica de Chile y Argentina (VII-X). Forma parte de los bosques de tipo valdiviano y de Nothofagus dombeyi. Exs. S. Teillier, J. Macaya, J. Delaunoy \& C. Bonnemaison 7330 (CONC).

\section{BERBERIDACEAE}

Berberis darwinii Hook.

michai Nanofanerófito. Endémica de Chile y Argentina (VII-XI). Crece en sitios alterados del bosque de tipo valdiviano y de Nothofagus dombeyi. Exs. S. Teillier, J. Delaunoy \& C. Bonnemaison 7199 (CONC).
Berberis microphylla G.Forst.

calafate Nanofanerófito. Endémica de Chile y Argentina (VI-XII). Crece en el bosque de Nothofagus dombeyi, en el de Nothofagus antarctica y en el matorral subandino. Exs. S. Teillier, J. Delaunoy \& C. Bonnemaison 6598, 7197, 7198 (CONC).

Berberis serratodentata Lechl.

Nanofanerófito. Endémica de Chile y Argentina (IX-XI). Crece en el bosque de Nothofagus pumilio, poco frecuente. Exs. S. Teillier, J. Delaunoy \& C. Bonnemaison 6595 (CONC).

Berberis trigona Kunze ex Poepp. Endl.

Nanofanerófito. Endémica de Chile y Argentina (VIII-X). Crece en los bosques de Nothofagus dombeyi y de Nothofagus antarctica, poco frecuente. Exs. S. Teillier, J. Delaunoy \& C. Bonnemaison 6596, 6597, 7196 (CONC).

\section{Bignoniaceae}

Campsidium valdivianum (Phil.) Skottsb. pilpilvoqui blanco Megafanerófito, trepador. Endémica de Chile y Argentina (VII$\mathrm{XI}$ ). Crece en el bosque de tipo valdiviano; poco frecuente. Exs. S. Teillier, J. Delaunoy \& C. Bonnemaison 7193 (CONC).

\section{BoraginACEAE}

Echium vulgare L. lengua de gato Terófito. Alóctona, asilvestrada (IV-XII), originaria de Europa y Asia. Crece en sitios alterados como praderas, orillas de caminos y de senderos. Exs. S. Teillier, J. Delaunoy \& C. Bonnemaison 6734 (CONC).

\section{Myosotis scorpioides L.}

Hemicriptófito en roseta. Alóctona asilvestrada (IX-XII), originaria de Europa y Asia. Crece en sitios húmedos, escasa. Exs. S. Teillier, J. Delaunoy \& C. Bonnemaison 6785 (CONC).

Phacelia secunda J.F.Gmel. cuncuna Hemicriptófito escapífero. Endémica de Chile y Argentina; en el país crece en todas las regiones. Crece en sitios arenosos, escasa. Exs. S. Teillier, J. Delaunoy \& C. Bonnemaison 6825 (CONC).

BrassicaceAe (Cruciferae)

Cardamine cordata Barnéoud berro Hemicriptófito en roseta. Endémica de Chile y Argentina (IV-X). Crece en el matorral subandino. Exs. S. Teillier, J. Delaunoy \& C. Bonnemaison 7478, 7479, 7481, 7485 (CONC).

Cardamine tenuirostris Hook. \& Arn. berro Hemicriptófito en roseta. Endémica de Chile y Argentina (IX-X). Crece en el margen de las quebradas y de los ríos. Exs. S. Teillier, A. Marticorena, J. Macaya \& C. Bonnemaison 7372, 7480 (CONC).

Cardamine variabilis Phil.

berro Hemicriptófito en roseta. Endémica de Chile y Argentina (VIIIXI). Crece en sitios húmedos. Exs. S. Teillier, J. Delaunoy \& C. Bonnemaison 7482, 7483 (CONC).

Cardamine vulgaris Phil. berro Hemicriptófito en roseta. Endémica de Chile y Argentina (V-XII). Crece en sitios húmedos y algo sombríos. Exs. S. Teillier, J. Delaunoy \& C. Bonnemaison 7161, 7484 (CONC). 


\section{Buddlejaceae}

Buddleja globosa Hope

matico

Mega o nanofanerófito. Endémica de Chile y Argentina (V-X). Crece en los claros del bosque de tipo valdiviano y de Nothofagus dombeyi y Nothofagus alpina, frecuente. Exs. S. Teillier, J. Delaunoy \& C. Bonnemaison 6591 (CONC).

\section{Calceolariaceae}

\section{Calceolaria crenatiflora Cav.}

Hemicriptófito en roseta. Endémica de Chile y Argentina (IX-XIV). Crece en los bosques de tipo valdiviano, pero es más frecuente en los de Nothofagus dombeyi. Exs. S. Teillier, J. Delaunoy \& C. Bonnemaison 6679 (CONC).

Calceolaria dentata Ruiz \& Pav. ssp. araucana (Phil.) C.Ehrhart Caméfito arbusto. Subespecie endémica de Chile y Argentina (VIII-X). Crece en los taludes del río Pillanleufú, poco frecuente. Exs. S. Teillier, J. Delaunoy \& C. Bonnemaison 6678 (CONC); S. Teillier, A. Marticorena, J. Macaya \& C. Bonnemaison 7371 (CONC).

\section{Calceolaria filicaulis $\mathrm{Clos}$}

Hemicriptófito en roseta. Endémica de Chile y Argentina (VII-X). Crece en sitios húmedos del bosque de Nothofagus pumilio y en el matorral subandino. Exs. S. Teillier, J. Delaunoy \& C. Bonnemaison 6676, 6677 (CONC).

Calceolaria tenella Poepp. \& Endl.

Hemicriptófito en roseta. Endémica de Chile y Argentina (VIIIXII). Crece en sitios muy húmedos, en los bosques de tipo valdiviano y de Nothofagus dombeyi; poco frecuente. Exs. S. Teillier, J. Delaunoy \& C. Bonnemaison 6675 (CONC).

\section{Calceolaria valdiviana Phil.}

Hemicriptófito en roseta. Endémica de Chile y Argentina (VIII-X). Crece en los bosques de Nothofagus dombeyi y de Nothofagus antarctica, prefiere claros y taludes. Exs. S. Teillier, J. Delaunoy \& C. Bonnemaison 6674 (CONC).

\section{Callitrichaceae}

Callitriche lechleri (Hegelm.) Fassett. var. berteroana (Hegelm.) Fassett. huenchecó Hidrófito. Subespecie endémica de Chile y Argentina, su distribución en el país está mal conocida; es la primera vez que se determina al sur de la Región de O’Higgins. Crece en lagunas o pequeños charcos. Exs. S. Teillier, J. Delaunoy \& C. Bonnemaison 6672 (CONC).

\section{Campanulaceae}

Legenere valdiviana (Phil.) E.Wimm.

Terófito. Especie endémica de Chile y Argentina (XIV-X). Crece solamente en la laguna Trarolafquén, en sitios inundables. Exs. S. Teillier, J. Macaya, J. Delaunoy \& C. Bonnemaison, 7231 (CONC); S. Teillier, A. Marticorena, J. Macaya \& C. Bonnemaison 7432 (CONC).

\section{CARYophyllaceae}

\section{Arenaria serpens Kunth}

Hemicriptófito repente. Especie nativa, no endémica (IV-X). Crece en la ribera de la laguna Trarolafquén y en otros sitios muy húmedos, incluso en algunos que se inundan periódicamente. Exs. S. Teillier, J. Delaunoy \& C. Bonnemaison 6616 (CONC).

\section{Cerastium arvense $\mathrm{L}$.}

Hemicriptófito escapífero. Alóctona asilvestrada (IV-XII) proveniente de Europa y Asia. Crece en el matorral subandino. Exs. S. Teillier, J. Delaunoy \& C. Bonnemaison 6468, 6669 (CONC).

Cerastium fontanum Baumg. ssp. vulgare (Hartmann) Greuter \& Burdet

Hemicriptófito en roseta. Alóctona asilvestrada (IV-XII), proveniente de Europa y Asia. Crece en ambientes perturbados, sea en praderas, bordes de caminos o claros de bosque. Exs. S. Teillier, J. Delaunoy \& C. Bonnemaison 6467, 7192 (CONC).

Petrorhagia dubia (Raf.) G.López \& Romo

Terófito. Especie alóctona asilvestrada (V-XII), con origen en Europa mediterránea. Se la observó sólo en praderas muy alteradas. Exs. S. Teillier, J. Delaunoy \& C. Bonnemaison 6495 (CONC).

\section{Sagina procumbens L.}

Terófito. Alóctona asilvestrada (VIII-XII), con origen en Europa y Asia. Crece en sitios húmedos y algo sombríos. Exs. S. Teillier, J. Delaunoy \& C. Bonnemaison 6466, 7546 (CONC).

Silene andicola Cav.

Hemicriptófito en roseta. Endémica de Chile y Argentina (VIII-X). Forma parte de la vegetación del matorral andino, escasa. Exs. S. Teillier, A. Marticorena, J. Macaya \& C. Bonnemaison 7382 (CONC).

\section{Spergula arvensis L.}

Terófito. Alóctona asilvestrada (IV-XII), con origen en Europa y Asia. Escasa en el área, crece en sitios alterados de la pampa Bandurrias. Exs. S. Teillier, J. Delaunoy \& C. Bonnemaison 7403 (CONC).

Stellaria arvalis Fenzl ex F.Phil. quilloiquilloi Hemicriptófito escapífero. Endémica de Chile y Argentina (VIIXII). Crece en los claros de los bosques de tipo valdiviano, de Nothofagus dombeyi y de Nothofagus antarctica. Exs. S. Teillier, J. Delaunoy \& C. Bonnemaison 6469, 6837 (CONC); S. Teillier, J. Macaya, J. Delaunoy \& C. Bonnemaison 7304 (CONC).

\section{Celastraceae}

Maytenus boaria Molina maitén Megafanerófito. Nativa no endémica (III-XII). Crece a la orilla de los ríos y los esteros. Exs. S. Teillier, J. Delaunoy \& C. Bonnemaison 6803, 7183 (CONC).

Maytenus disticha (Hook. f.) Urb.

Nanofanerófito. Endémica de Chile y Argentina (VII-XII). Forma parte del sotobosque de Nothofagus pumilio. Exs. S. Teillier, J. Delaunoy \& C. Bonnemaison 7181 (CONC); S. Teillier, J. Macaya, J. Delaunoy \& C. Bonnemaison 7262 (CONC).

Maytenus magellanica (Lam.) Hook. f. leña dura Megafanerófito. Endémica de Chile y Argentina (VII-XII). Forma parte del bosque de Nothofagus dombeyi y de Nothofagus pumilio. Exs. S. Teillier, J. Delaunoy \& C. Bonnemaison 6801, 6802, 7182 (CONC). 


\section{Convolvulaceae}

Cuscuta pauciflora Phil.

cabello de ángel

Terófito, parásito. Endémica de Chile y Argentina; distribución en nuestro país poco conocida, ha sido recolectada en las regiones del Maule y de Los Lagos. Crece en la laguna Trarolafquén, tiene como huésped principal a Azorella trifoliolata, aunque se la observó también sobre Arenaria serpens. Exs. S. Teillier, J. Delaunoy \& C. Bonnemaison 6659 (CONC); S. Teillier, A. Marticorena, J. Macaya \& C. Bonnemaison 7430 (CONC).

Coriaceae

Coriaria ruscifolia $\mathrm{L}$.

deu

Hemicriptófito escapífero. Endémica de Chile y Argentina (VIIXI). Crece en los claros del bosque de Nothofagus dombeyi, en sitios próximos a ríos o quebradas. Exs. S. Teillier, J. Delaunoy \& C. Bonnemaison 6662 (CONC).

\section{CunOniaceae}

Caldcluvia paniculata (Cav.) D.Don.

tiaca

Megafanerófito. Endémica de Chile y Argentina (VII-XI). Forma parte del bosque de tipo valdiviano y es más común cerca de los cursos de agua. Exs. S. Teillier, J. Delaunoy \& C. Bonnemaison 6673 (CONC); S. Teillier, J. Macaya, J. Delaunoy \& C. Bonnemaison 7307, 7332, 7333 (CONC).

Eucryphia cordifolia Cav.

ulmo Megafanerófito. Endémica de Chile y Argentina (VII-XI). Crece en el bosque de tipo valdiviano, donde es una de las especies dominantes. Exs. S. Teillier, J. Delaunoy \& C. Bonnemaison 6712 (CONC).

Weinmannia trichosperma Cav.

tineo Megafanerófito. Especie endémica de Chile y Argentina (VIIXII). Forma parte de los bosques de tipo valdiviano y de los de Nothofagus dombeyi. Exs. S. Teillier, J. Delaunoy \& C. Bonnemaison 6908 (CONC).

\section{DesfontainiaCEAE}

Desfontainia fulgens D.Don

taique Nanofanerófito. Especie nativa no endémica (VII-XII). Forma parte de los bosques de Nothofagus dombeyi. Exs. S. Teillier, J. Delaunoy \& C. Bonnemaison 6654 (CONC).

Elaeocarpaceae

Aristotelia chilensis (Molina) Stuntz maqui Megafanerófito. Especie nativa no endémica (IV-XI). Crece en sitios alterados del bosque valdiviano. Exs. S. Teillier, J. Delaunoy \& C. Bonnemaison 7202 (CONC); S. Teillier, J. Macaya, J. Delaunoy \& C. Bonnemaison 7319 (CONC).

\section{EMPETRACEAE}

Empetrum rubrum Vahl ex Willd.

brecillo Nanofanerófito. Endémica de Chile y Argentina (V-XII). Crece en el matorral subandino, en el margen de una pradera húmeda de altura, poco frecuente. Exs. S. Teillier, J. Delaunoy \& C. Bonnemaison 6278, 6279 (CONC).

ERICACEAE

Gaultheria antarctica Hook. f.

Caméfito en espaldera. Endémica de Chile y Argentina (XIV-XII).
Crece en el matorral subandino en sitios húmedos, escasa. Exs. S. Teillier, J. Delaunoy \& C. Bonnemaison 6707 (CONC); S. Teillier, J. Macaya, J. Delaunoy \& C. Bonnemaison 7441 (CONC).

Gaultheria phillyreifolia (Pers.) Sleumer

Nanofanerófito. Endémica de Chile y Argentina (VI-XII). Crece en los bosques de Nothofagus dombeyi donde es uno de los arbustos dominantes en los claros que se forman con la tala. Exs. S. Teillier, J. Delaunoy \& C. Bonnemaison 6706, 7186 (CONC).

Gaultheria poeppigii DC.

chaura

Nanofanerófito y caméfito en espaldera. Endémica de Chile y Argentina (VII-XI). Crece en los bosques de Nothofagus antarctica, de Nothofagus pumilio y en el matorral subandino, donde es una de las especies dominantes. Exs. S. Teillier, J. Delaunoy \& C. Bonnemaison 6704, 6705, 7185 (CONC).

Gaultheria pumila (L.f.) D.J.Middleton var. pumila Caméfito en espaldera. Endémica de Chile y Argentina; la variedad típica crece entre las regiones de Los Ríos y de Magallanes. Forma parte de la vegetación andina, en sitios abiertos y húmedos. Exs. S. Teillier, J. Delaunoy \& C. Bonnemaison 6701, 6702 (CONC).

Gaultheria tenuifolia (Phil.) Sleumer

Nanofanerófito. Endémica de Chile y Argentina (VII-X). Crece en el margen del bosque de Nothofagus pumilio, donde se observó un sólo grupo de ejemplares en el camino a Puerto Pirehueico, a unos 1.300 msnm. Exs. S. Teillier, J. Delaunoy \& C. Bonnemaison 6698 (CONC); S. Teillier, J. Macaya, J. Delaunoy \& C. Bonnemaison 7263 (CONC).

\section{EsCALLONIACEAE}

Escallonia alpina Poepp. ex DC

Nanofanerófito. Endémica de Chile y Argentina (V-XII). Crece tanto en el bosque de Nothofagus pumilio, como en el matorral subandino. Exs. S. Teillier, J. Delaunoy \& C. Bonnemaison 6717 (CONC); S. Teillier, J. Macaya, J. Delaunoy \& C. Bonnemaison 7242 (CONC).

Escallonia leucantha J.Remy luncillo Nanofanerófito. Endémica de Chile y Argentina (VII-X). Se la observó sólo en el bosque de Nothofagus dombeyi. Exs. S. Teillier, J. Macaya, J. Delaunoy \& C. Bonnemaison 7265 (CONC).

\section{Escallonia rosea Griseb.}

Nanofanerófito. Endémica de Chile y Argentina (VII-XII). Se la observó en el valle del Pillanleufú, donde crece en sitios inundables. Exs. S. Teillier, J. Delaunoy \& C. Bonnemaison 6905 (CONC); S. Teillier, J. Macaya, J. Delaunoy \& C. Bonnemaison 7284 (CONC).

Escallonia rubra (Ruiz \& Pav.) Pers. ñipa Nanofanerófito. Endémica de Chile y Argentina (V-XII). Crece en ambientes húmedos, como bordes de ríos y esteros. Exs. S. Teillier, J. Delaunoy \& C. Bonnemaison 6716, 7189 (CONC).

Escallonia virgata (Ruiz \& Pav.) Pers.

meki Nanofanerófito. Endémica de Chile y Argentina (VII-XII). Crece en sitios muy húmedos e inundables como en la laguna Trarolafquén, en la pampa Pilmaiquén, donde forma una población 
densa en torno al espejo de agua. Exs. S. Teillier, J. Delaunoy \& C. Bonnemaison 6713, 6715 (CONC).

\section{EUPHORBIACEAE}

Dysopsis glechomoides (A. Rich.) Müll. Arg.

Hemicriptófito repente. Nativa no endémica (IV,VIII,XII). Forma parte del sotobosque de Nothofagus dombeyi. Exs. S. Teillier, J. Delaunoy \& C. Bonnemaison 6643 (CONC).

FABACEAE (Papilionaceae)

Adesmia emarginata Clos

paramela

Nanofanerófito. Endémica de Chile y Argentina (VII-XI). Forma parte del matorral subandino. Exs. S. Teillier, J. Delaunoy \& C. Bonnemaison 6626, 6627, 6628, 6629 (CONC).

\section{Adesmia longipes Phil.}

Hemicriptófito escapífero. Endémica de Chile y Argentina (RM$\mathrm{X}$ ). Forma parte del matorral andino, es una de las especies dominantes en el volcán Mocho-Choshuenco. Exs. S. Teillier, J. Delaunoy \& C. Bonnemaison 6625 (CONC); S. Teillier, A. Marticorena, J. Macaya \& C. Bonnemaison 7435 (CONC).

\section{Adesmia retusa Griseb.}

Hemicriptófito repente. Endémica de Chile y Argentina (XIV-X). Crece en el matorral subandino. Exs. S. Teillier, J. Delaunoy \& C. Bonnemaison 6906 (CONC); S. Teillier, A. Marticorena, J. Macaya \& C. Bonnemaison 7390 (CONC).

Cytisus scoparius (L.) Link retama

Nanofanerófito. Alóctona asilvestrada (VII-X), de origen europeo. Escasa, se encontraron algunos ejemplares en el camino a la pampa Pilmaiquén en un claro del bosque de Nothofagus dombeyi. Exs. S. Teillier, J. Delaunoy \& C. Bonnemaison 6656 (CONC).

Lathyrus magellanicus Lam.

arvejilla

Hemicriptófito trepador. Endémica de Chile y Argentina (V-XII). Crece en el bosque de Nothofagus antarctica. Exs. S. Teillier, J. Macaya, J. Delaunoy \& C. Bonnemaison 6750 (CONC).

Lotus pedunculatus Cav. alfalfa chilota Hemicriptófito escapífero. Alóctona asilvestrada (VII-XI), originaria de Eurasia. Crece en sitios alterados como las praderas, los bordes de los caminos y los claros de los bosques talados donde es una de las especies más abundantes. Exs. S. Teillier, J. Delaunoy \& C. Bonnemaison 7276 (CONC).

Trifolium dubium Sibth. trébol

Hemicriptófito repente. Alóctona asilvestrada (V-XII), originaria de Europa. Crece en sitios alterados, como las praderas y los bordes de los caminos, en sitios que se encharcan. Exs. S. Teillier, J. Delaunoy \& C. Bonnemaison 6922 (CONC).

Trifolium pratense $\mathrm{L}$.

trébol rosado Hemicriptófito repente. Alóctona asilvestrada (IV-XII), originaria de Eurasia. Crece en las praderas y en los bordes de los caminos. Exs. S. Teillier, J. Delaunoy \& C. Bonnemaison 6920, 6921 (CONC).

\section{Trifolium repens $\mathrm{L}$.}

Hemicriptófito repente. Alóctona asilvestrada (IV-XII), originaria de Eurasia. Crece en sitios alterados como las praderas y en los bordes de los caminos; abundante en las vegas, siendo una especie más higrófila que las anteriores. Exs. S. Teillier, J. Delaunoy \& C. Bonnemaison 6919 (CONC).

\section{Ulex europaeus L.} espinillo Nanofanerófito. Alóctona asilvestrada (VIII-X), originaria de Europa. Crece sólo en la pampa Pilmaiquén en un claro del bosque de Nothofagus antarctica. No coleccionado.

Vicia magellanica Hook. f. arvejilla

Hemicriptófito trepador. Endémica de Chile y Argentina (IXXII). Crece en el bosque de Nothofagus antarctica (ñirre), en la pampa Pilmaiquén, escasa. Exs. S. Teillier, J. Delaunoy \& C. Bonnemaison 6913 (CONC); S. Teillier, J. Macaya, J. Delaunoy \& C. Bonnemaison 7236 (CONC).

Vicia nigricans Hook. \& Arn. Hemicriptófito trepador. Endémica de Chile y Argentina (V-XI). Crece de preferencia en los bosques de Nothofagus dombeyi. Exs. S. Teillier, J. Delaunoy \& C. Bonnemaison 6911, 6912 (CONC).

\section{FRANCOACEAE}

Francoa appendiculata Cav. llaupangue Hemicriptófito en roseta. Endémica de Chile (V-X). Crece en el bosque de tipo valdiviano y en el de Nothofagus dombeyi en claros y taludes húmedos, escasa. Exs. S. Teillier, J. Delaunoy \& C. Bonnemaison 6708 (CONC); S. Teillier, J. Macaya, J. Delaunoy \& C. Bonnemaison 7303 (CONC).

\section{GeraniaceaE}

Geranium core-core Steud. core core Hemicriptófito repente. Nativa no endémica, distribuida en todas las regiones del país. Crece en las praderas húmedas, inclusive en aquéllas más alteradas, poco frecuente. Exs. S. Teillier, J. Delaunoy \& C. Bonnemaison 6695 (CONC); S. Teillier, J. Macaya, J. Delaunoy \& C. Bonnemaison 7257 (CONC).

Geranium magellanicum Hook. f.

Hemicriptófito escapífero. Endémica de Chile y Argentina (IXXII). Crece en las praderas y en los claros de los bosques. Exs. S. Teillier, J. Delaunoy \& C. Bonnemaison 6694, 6694-A (CONC).

\section{Geranium sessiliflorum Cav.}

Hemicriptófito en roseta. Especie nativa no endémica, distribuida en todas las regiones del país. Crece en las praderas andinas entre los 1100 y los 1500 msnm. Exs. S. Teillier, J. Delaunoy \& C. Bonnemaison 6692, 6693 (CONC).

\section{Gesneriaceae}

Asteranthera ovata (Cav.) Hanst. estrellita Hemicriptófito repente y epífito arborícola. Endémica de Chile y Argentina (VII-XII). Se encuentra en los bosques de Nothofagus dombeyi. Exs. S. Teillier, J. Delaunoy \& C. Bonnemaison 6612 (CONC).

Mitraria coccinea Cav.

botellita Nanofanerófito y epífito arborícola. Endémica de Chile y Argentina ((IV) VII-XII). Se encuentra en el bosque de tipo valdiviano, menos frecuente en los de Nothofagus dombeyi. Exs. S. Teillier, J. 
Delaunoy \& C. Bonnemaison 7316 (CONC); S. Teillier, J. Macaya, J. Delaunoy \& C. Bonnemaison 6791 (CONC).

Sarmienta scandens (J.D.Brandis ex Molina) Pers. medallita Epífito arborícola. Endémica de Chile y Argentina ((IV) VII$\mathrm{XI})$. Se encuentra sólo en los bosques de tipo valdiviano, poco frecuente. Exs. S. Teillier, J. Delaunoy \& C. Bonnemaison 6857 (CONC).

\section{GRISELINIACEAE}

Griselinia ruscifolia (Clos) Ball lilinquén Nanofanerófito y epífito arborícola. Especie nativa no endémica (XIV-XII). Crece en los bosques montanos de Nothofagus dombeyi y Nothofagus alpina, poco frecuente. Exs. S. Teillier, J. Delaunoy \& C. Bonnemaison 6684, 6685 (CONC).

Grossulariaceae

Ribes cucullatum Hook. \& Arn. parilla Nanofanerófito. Endémica de Chile y Argentina (V-XII). Crece en el matorral subandino sobre los $1.000 \mathrm{msnm}$. Exs. S. Teillier, J. Delaunoy \& C. Bonnemaison 6866, 6867 (CONC).

\section{Ribes magellanicus Poir.}

Nanofanerófito. Endémica de Chile y Argentina (VI-XII). Crece en los claros de los bosques de Nothofagus dombeyi y Nothofagus antactica. Exs. S. Teillier, J. Delaunoy \& C. Bonnemaison 6864, 6865, 7162, 7164 (CONC); S. Teillier, J. Macaya, J. Delaunoy \& C. Bonnemaison 7225 (CONC).

\section{Ribes nitidissima Neger}

Nanofanerófito. Endémica de Chile y Argentina (VIII-XIV). Crece en el bosque de lenga y en el matorral subandino, poco frecuente. Exs. S. Teillier, J. Delaunoy \& C. Bonnemaison 7407 (CONC); S. Teillier, J. Macaya, J. Delaunoy \& C. Bonnemaison 7477 (CONC).

\section{GunNeraceae}

Gunnera magellanica Lam.

Hemicriptófito en roseta. Endémica de Chile y Argentina (V-XII). Crece en algunos humedales en presencia de agua superficial; escasa, se la observó en el el volcán Mocho-Choshuenco, en el valle del Pillanleufú (655 msnm) y en el camino a Puerto Pirehueico (1.400 msnm). Exs. S. Teillier, J. Delaunoy \& C. Bonnemaison 6682, 6683 (CONC); S. Teillier, J. Macaya, J. Delaunoy \& C. Bonnemaison 7256 (CONC).

Gunnera tinctoria (Molina) Mirb.

pangue

Hemicriptófito escapífero. Endémica de Chile y Argentina (IVXII). Crece en sitios con humedad permanente y prefiere los suelos arenosos donde alcanza su mayor tamaño. Exs. S. Teillier, J. Macaya, J. Delaunoy \& C. Bonnemaison 7272, 7424 (CONC).

\section{HALORAGACEAE \\ Myriophyllum quitense Kunth}

Hidrófito. Especie nativa, no endémica, crece en todas las regiones del país. Se encontró solamente en el río Pillanleufú, en lagunas someras. Exs. S. Teillier, J. Macaya, J. Delaunoy \& C. Bonnemaison 7278 (CONC).

Hydrangeaceae

Hydrangea serratifolia (Hook. \& Arn.) F.Phil.
Fanerófito trepador. Endémica de Chile y Argentina (V-XI). Crece en el bosque de tipo valdiviano; poco frecuente en el de Nothofagus dombeyi. Exs. S. Teillier, J. Delaunoy \& C. Bonnemaison 6737 (CONC).

\section{HYPERICACEAE}

Hypericum caespitosum Cham. \& Schltdl.

Hemicriptófito repente. Endémica de Chile (VII-X). Se encontró en un sitio muy húmedo en el margen de un bosque de Nothofagus dombeyi, muy escasa. Exs. S. Teillier, J. Delaunoy \& C. Bonnemaison 6738 (CONC).

Hypericum perforatum $\mathrm{L}$.

hierba de San Juan Hemicriptófito escapífero. Alóctona asilvestrada (RM-X), proveniente de Eurasia. Se encuentra en las praderas y otros sitios alterados, invasiva y abundante. Exs. S. Teillier, J. Delaunoy \& C. Bonnemaison 6739 (CONC).

LAmiaceAe (Labiatae)

Prunella vulgaris L.

hierba mora

Hemicriptófito repente. Alóctona asilvestrada (V-XII). Crece en sectores alterados de los bosques, también en las praderas y los márgenes de los caminos y los senderos. Exs. S. Teillier, J. Delaunoy \& C. Bonnemaison 6808, 6810 (CONC).

\section{LARDIZABALACEAE}

Boquila trifoliolata (DC.) Decne. pilpilvoqui Fanerófito trepador. Endémica de Chile y Argentina (VII-X). Se encuentra tanto en el bosque de tipo valdiviano como en los de Nothofagus dombeyi. Exs. S. Teillier, J. Macaya, J. Delaunoy \& C. Bonnemaison 7286 (CONC).

Lauraceae

Persea lingue $\mathrm{Nees}$ lingue Megafanerófito. Endémica de Chile y Argentina (V-X). Forma parte del bosque de tipo valdiviano, donde es uno de los árboles más frecuentes. Exs. S. Teillier, J. Delaunoy \& C. Bonnemaison 7312 (CONC).

\section{LINACEAE}

Linum bienne Mill. lino Hemicriptófito bienal. Alóctona asilvestrada (III-X). Crece en las praderas antropogénicas. Exs. S. Teillier, J. Delaunoy \& C. Bonnemaison 6754 (CONC); S. Teillier, A. Marticorena, J. Macaya \& C. Bonnemaison 7402 (CONC).

LORANTHACEAE

Tristerix corymbosus (L.) Kuijt quintral Epífito arborícola, hemiparásito. Endémica de Chile y Argentina (IV-X). Se encontró solamente en el bosque de tipo valdiviano; escasa. Exs. S. Teillier, J. Delaunoy \& C. Bonnemaison 6918 (CONC).

\section{MisodendRaceAe}

Misodendrum brachystachyum DC.

Epífito arborícola, hemiparásito. Endémica de Chile y Argentina (VII-XII). Tiene como hospedero a Nothofagus dombeyi; muy escasa. Exs. S. Teillier, J. Delaunoy \& C. Bonnemaison 7178 (CONC). 
Misodendrum linearifolium DC

cabello de ángel Epífito arborícola, hemiparásito. Endémica de Chile y Argentina (V-XII). Tiene como hospederos a Nothofagus antarctica, $N$. dombeyi y N. obliqua, poco frecuente. Exs. S. Teillier, J. Delaunoy \& C. Bonnemaison 6797, 6798 (CONC).

\section{Misodendrum oblongifolium DC.}

Epífito arborícola, hemiparásito. Endémica de Chile y Argentina (VII-XI). Su hospedero más frecuente es Nothofagus pumilio, poco frecuente. Exs. S. Teillier, J. Delaunoy \& C. Bonnemaison 6794 (CONC); S. Teillier, J. Macaya, J. Delaunoy \& C. Bonnemaison 7378 (CONC).

Misodendrum punctulatum Banks ex DC. injerto Epífito arborícola, parásito. Endémica de Chile y Argentina (VIIIXII). Sus hospederos son Nothofagus antarctica y Nothofagus dombeyi, muy frecuente. Exs. S. Teillier, J. Delaunoy \& C. Bonnemaison 6792, 6793, 7179, 7180 (CONC).

\section{Montiaceae}

Calandrinia colchaguensis Barnéoud

Hemicriptófito en roseta. Endémica de Chile y Argentina (VI-X). Crece en las praderas húmedas de altura, escasa. Exs. S. Teillier, J. Delaunoy \& C. Bonnemaison 6680 (CONC)

\section{Montiopsis gayana (Barnéoud) D.I.Ford}

Hemicriptófito en roseta. Endémica de Chile y Argentina (IV-X). Crece en el matorral subandino. Exs. S. Teillier, J. Delaunoy \& C. Bonnemaison 6787, 6788, 7177 (CONC).

\section{MyrTACEAE}

Amomyrtus luma (Molina) D.Legrand \& Kausel luma Megafanerófito. Endémica de Chile y Argentina (VI-XI). Crece en el bosque de tipo valdiviano y en los de Nothofagus dombeyi; una de las especies más frecuentes en los sitios talados. Exs. S. Teillier, J. Macaya, J. Delaunoy \& C. Bonnemaison 7314, 7334 (CONC).

\section{Luma apiculata (DC.) Burret}

arrayán Megafanerófito. Endémica de Chile y Argentina (IV-XI). Crece en el bosque de tipo valdiviano y en las praderas antropogénicas; una de las especies más frecuentes en los sitios talados. Exs. S. Teillier, J. Delaunoy \& C. Bonnemaison 6759 (CONC); S. Teillier, J. Macaya, J. Delaunoy \& C. Bonnemaison 7345 (CONC).

Myrceugenia chrysocarpa (O.Berg) Kausel luma blanca Nanofanerófito. Endémica de Chile y Argentina (VII-X). Se encuentra en los bosques de Nothofagus pumilio. Exs. S. Teillier, J. Delaunoy \& C. Bonnemaison 6782, 6783, 6784 (CONC).

\section{Myrceugenia ovata (Hook. \& Arn.) O.Berg var. nannophylla} (Burret) Landrum huillipeta Nanofanerófito. Subespecie endémica de Chile y Argentina (VII-X). Se encontró una sola vez en un bosque de Nothofagus dombeyi (coigüe). Exs. S. Teillier, J. Macaya, J. Delaunoy \& C. Bonnemaison 7267, 7518, 7545 (CONC).

Myrceugenia planipes (Hook. \& Arn.) O.Berg

pitra Megafanerófito. Endémica de Chile y Argentina (VIII-XI). Crece en el bosque de tipo valdiviano, una de las especies más frecuentes. Exs. S. Teillier, J. Delaunoy \& C. Bonnemaison 7176 (CONC); S.
Teillier, J. Macaya, J. Delaunoy \& C. Bonnemaison 7331 (CONC).

Ugni molinae Turcz.

murtilla Nanofanerófito. Endémica de Chile y Argentina (VII-XII). Crece en el bosque de tipo valdiviano. Exs. S. Teillier, J. Macaya, J. Delaunoy \& C. Bonnemaison 7423 (CONC).

NothofagaceAe

Nothofagus alpina (Poepp. \& Endl.) Oerst. raulí Megafanerófito. Endémica de Chile y Argentina (VII-X). Crece en los bosques Nothofagus dombeyi ubicados a mediana altitud; donde es codominante. Exs. S. Teillier, J. Delaunoy \& C. Bonnemaison 7261 (CONC).

Nothofagus antarctica (G.Forst.) Oerst. ñirre Megafanerófito. Endémica de Chile y Argentina (VII-XII). Crece como dominante en los bosques de la pampa Pilmaiquén, entre 700 y $800 \mathrm{msnm}$, probablemente un área de frecuentes inversiones térmicas, con fríos intensos y cubierta por nieve durante largo tiempo. Exs. S. Teillier, J. Delaunoy \& C. Bonnemaison 6770, 6771, 6772 (CONC).

Nothofagus dombeyi (Mirb.) Oerst.

coihue Megafanerófito. Endémica de Chile y Argentina (VI-XI). Crece como especie dominante en los bosques montanos y también está presente en el bosque valdiviano; es el árbol más abundante en la reserva. Exs. S. Teillier, J. Delaunoy \& C. Bonnemaison 6769 (CONC); S. Teillier, J. Macaya, J. Delaunoy \& C. Bonnemaison 7320 (CONC).

Nothofagus obliqua (Mirb.) Oerst

hualle, roble Megafanerófito. Endémica de Chile y Argentina (VI-X). Crece en el bosque de tipo valdiviano, en los sectores ubicados a menor altitud. Exs. S. Teillier, J. Delaunoy \& C. Bonnemaison 6768 (CONC); S. Teillier, J. Macaya, J. Delaunoy \& C. Bonnemaison 7329 (CONC).

Nothofagus pumilio (Poepp. \& Endl.) Krasser lenga Megafanerófito. Endémica de Chile y Argentina (VII-XII). Crece como dominante en los bosques montanos que constituyen el límite superior de la distribución de los árboles en la reserva. Exs. S. Teillier, J. Delaunoy \& C. Bonnemaison 6767, 7163 (CONC).

\section{ONAGRACEAE}

Epilobium australe Poepp. \& Hausskn. ex Hausskn.

Hemicriptófito escapífero. Endémica de Chile y Argentina (VIXII). Crece en los márgenes de los humedales o en sitios anegados. Exs. S. Teillier, J. Delaunoy \& C. Bonnemaison 6725, 6727 (CONC).

\section{Epilobium ciliatum Raf.}

Hemicriptófito escapífero. Nativa no endémica (III-XII). Crece en los márgenes de los humedales o en sitios anegados. Exs. S. Teillier, J. Delaunoy \& C. Bonnemaison 6726 (CONC)

Epilobium densifolium Hausskn.

Hemicriptófito escapífero. Endémica de Chile y Argentina (VIX). Crece en los márgenes de los humedales o sitios anegados, en sitios de altura. Exs. S. Teillier, A. Marticorena, J. Macaya \& C. Bonnemaison 7379, 7476, 7526 (CONC). 
Epilobium glaucum Phil.

Hemicriptófito escapífero. Endémica de Chile y Argentina (IVXII). Crece en sitios húmedos, algo arenosos; en el río Pillanleufú. Exs. S. Teillier, J. Delaunoy \& C. Bonnemaison 6724 (CONC); S. Teillier, J. Macaya, J. Delaunoy \& C. Bonnemaison 7280 (CONC).

\section{Epilobium puberulum Hook. \& Arn.}

Hemicriptófito escapífero. Endémica de Chile y Argentina (V-XI). Crece en sitios húmedos, incluso en praderas alteradas y bordes de caminos, poco frecuente. Exs. S. Teillier, J. Delaunoy \& C. Bonnemaison 6722, 6723 (CONC).

Fuchsia magellanica Lam. chilco Nanofanerófito. Endémica de Chile y Argentina (V-XII). Crece en sitios húmedos como los ríos, las quebradas y los bordes de las lagunas. Exs. S. Teillier, J. Macaya, J. Delaunoy \& C. Bonnemaison 7188, 7221, 7266 (CONC).

Oenothera stricta Ledeb. ex Link ssp. altissima W.Dietr. don Diego de la noche Hemicriptófito escapífero. Subespecie endémica de Argentina; esta cita amplía su distribución a nuestro país. Se la observó sólo en el valle del Pillanleufú, muy escasa. Exs. S. Teillier, J. Delaunoy \& C. Bonnemaison 6836 (CONC).

\section{OXALIDACEAE}

Oxalis magellanica G.Forst.

ojo de agua

Hemicriptófito en roseta. Endémica de Chile y Argentina (IXXII). Se la observó sólo en la parte más alta $(1.460 \mathrm{msnm})$ del camino a Puerto Pirehueico, en un afloramiento de humedad. No se coleccionó.

Oxalis aff. nahuelhuapiensis Speg.

Hemicriptófito en roseta. Endémica de Chile y Argentina; en el país se la ha encontrado solamente en la Región de La Araucanía. Se la observó una sola vez en el volcán Mocho-Choshuenco, a unos $1.600 \mathrm{~m}$ de altitud. Exs. S. Teillier, J. Delaunoy \& C. Bonnemaison 6879 (CONC).

\section{Oxalis valdiviensis Barnéoud}

Hemicriptófito en roseta. Endémica de Chile y Argentina (VI-X). Crece en los taludes de los caminos, en los lechos de las quebradas y de los ríos; en la pampa Pilmaiquén y en el valle del río Pillanleufú. Exs. S. Teillier, J. Delaunoy \& C. Bonnemaison 6880 (CONC).

\section{Phytolaccaceae}

Ercilla syncarpellata Nowicke coralillo Fanerófito trepador. Endémica de Chile (IX-X). Crece en el bosque valdiviano, escasa. Exs. S. Teillier, J. Delaunoy \& C. Bonnemaison 6719 (CONC).

Plantaginaceae

Littorella australis Griseb. ex Benth. \& Hook. f.

Hidrófito. Endémica de Chile y Argentina (IX-XI). Crece solamente en la laguna Trarolafquén. Exs. S. Teillier, J. Macaya, J. Delaunoy \& C. Bonnemaison 7332, 7429 (CONC).

Plantago australis Lam. ssp. cumingiana (Fisch. \& C.A.Mey.) Rahn

Hemicriptófito en roseta. Subespecie endémica de Chile y
Argentina (V-XII). Crece en los claros de los bosques de tipo valdiviano, de Nothofagus dombeyi y de Nothofagus antarctica; también en senderos y praderas, húmedos. Exs. S. Teillier, J. Delaunoy \& C. Bonnemaison 6822 (CONC).

Plantago uniglumis Wallr. ex Walp.

Hemicriptófito en roseta. Endémica de Chile y Argentina (IV-XII). Se encontró solamente en un humedal de altura a unos $1.400 \mathrm{~m}$ de altitud. Exs. S. Teillier, J. Macaya, J. Delaunoy \& C. Bonnemaison 7396 (CONC).

Plantago lanceolata L.

siete venas Hemicriptófito en roseta. Alóctona asilvestrada (II-XII).Crece en las praderas antropogénicas y en los márgenes de los caminos. Exs. S. Teillier, J. Delaunoy \& C. Bonnemaison 7259 (CONC).

Plantago major L. llantén Hemicriptófito en roseta. Alóctona asilvestrada, se encuentra en todas las regiones del país. Crece en las praderas antropogénicas y en los márgenes de los caminos. S. Teillier, J. Delaunoy \& C. Bonnemaison 7520 (CONC).

\section{Polemoniaceae}

Collomia biflora (Ruiz \& Pav.) Brand

Terófito. Endémica de Chile y Argentina (V-XII). Crece sólo en el valle del río Pillanleufú, en suelos arenosos. Exs. S. Teillier, J. Macaya, J. Delaunoy \& C. Bonnemaison 7279 (CONC).

\section{Polygonaceae}

Muehlenbeckia hastulata (Sm.) I.M.Johnst. var. fascicularis (Meisn.) Brandbyge quilo Fanerófito trepador. Subespecie endémica de Chile y Argentina (V$\mathrm{X})$. Crece de preferencia en el bosque de tipo valdiviano. Exs. S. Teillier, J. Macaya, J. Delaunoy \& C. Bonnemaison 7246 (CONC).

Polygonum aviculare $\mathrm{L}$. pasto del pollo Terófito. Alóctona, asilvestrada en todas las regiones de Chile. Crece en sitios muy alterados como en el antiguo aserradero de la pampa Bandurrias. Exs. S. Teillier, J. Macaya, J. Delaunoy \& C. Bonnemaison 6821, 7339 (CONC).

Polygonum hydropiper L.

duraznillo Terófito. Alóctona asilvestrada (VIII-X). Crece en sitios húmedos alterados, se la observó en el aserradero antiguo en la pampa Bandurrias. Exs. S. Teillier, J. Macaya, J. Delaunoy \& C. Bonnemaison 7401 (CONC).

Polygonum persicaria L.

duraznillo Hemicriptófito rizomatoso. Alóctona asilvestrada (XV-XI). Crece en sitios húmedos alterados como los márgenes de los caminos, las praderas antropogénicas y pantanos en sitios abiertos. Exs. S. Teillier, J. Delaunoy \& C. Bonnemaison 6820 (CONC); S. Teillier, J. Macaya, J. Delaunoy \& C. Bonnemaison 7446 (CONC).

Rumex acetosella $\mathrm{L}$. vinagrillo Geófito rizomatoso. Alóctona, asilvestrada en todas las regiones del país. Crece en todo tipo de sitios alterados, menos en humedales; muy frecuente en praderas que fueron sobrepastoreadas. Exs. S. Teillier, J. Delaunoy \& C. Bonnemaison 7448 (CONC). 
Rumex crispus L.

romasa

Hemicriptófito escapífero. Alóctona asilvestrada (II-XII). Crece en sitios húmedos, alterados. Exs. S. Teillier, J. Delaunoy \& C. Bonnemaison 6861 (CONC).

\section{Rumex obtusifolius L.}

Hemicriptófito escapífero. Alóctona asilvestrada (II-XII). Crece en sitios húmedos, alterados. Exs. S. Teillier, J. Delaunoy \& C. Bonnemaison 7523 (CONC).

Primulaceae

Anagallis alternifolia Cav.

Hemicriptófito repente. Endémica de Chile y Argentina (II-XII). Crece en las praderas húmedas de la laguna Trarolafquén. Exs. S. Teillier, J. Delaunoy \& C. Bonnemaison 6619 (CONC).

Lysimachia sertulata Baudo

melilukue Hemicriptófito escapífero. Endémica de Chile y Argentina (VII-X). Crece en el bosque valdiviano, en sitios muy húmedos, al interior del bosque, también a la orilla de los ríos. Exs. S. Teillier, J. Delaunoy \& C. Bonnemaison 6765, 6766 (CONC); S. Teillier, J. Macaya, J. Delaunoy \& C. Bonnemaison 7306 (CONC).

Primula comberi W.W.Sm.

Hemicriptófito en roseta. Especie descrita en Argentina, en un sector de altura de la provincia de Neuquén; esta es la primera vez que se encuentra en Chile. En Huilo Huilo crece en un humedal de altura en el camino a Puerto Pirehueico, a unos $1.400 \mathrm{~m}$ de altitud. Exs. S. Teillier, J. Delaunoy \& C. Bonnemaison 6876 (CONC).

\section{Proteaceae}

Embothrium coccineum J.R.Forst. \& G.Forst. notro Megafanerófito y nanofanerófito. Endémica de Chile y Argentina (VII-XII). Crece en el bosque de Nothofagus dombeyi, en el de Nothofagus antractica y en los matorrales subandinos, donde es más frecuente como arbusto caducifolio. Exs. S. Teillier, J. Delaunoy \& C. Bonnemaison 6730, 6731 (CONC).

Gevuina avellana Molina

avellano

Megafanerófito. Endémica de Chile y Argentina (VII-X). Forma parte del bosque de tipo valdiviano y del de Nothofagus dombeyi. Exs. S. Teillier, J. Delaunoy \& C. Bonnemaison 6691 (CONC).

Lomatia dentata (Ruiz \& Pav.) R.Br.

piñol

Megafanerófito. Endémica de Chile y Argentina (V-X). Crece en el bosque de tipo valdiviano; poco frecuente. No se coleccionó.

Lomatia ferruginea (Cav.) R.Br. fuinque Megafanerófito. Endémica de Chile y Argentina (VII-XII). Forma parte del bosque de tipo valdiviano; frecuente en los sectores más húmedos. S. Teillier, J. Delaunoy \& C. Bonnemaison 7507 (CONC).

Lomatia hirsuta (Lam.) Diels

radal Megafanerófito. Especie nativa no endémica (IV-X). Crece en el bosque de Nothofagus antarctica. Exs. S. Teillier, J. Delaunoy \& C. Bonnemaison 6756 (CONC).

Ranunculaceae

Anemone antucensis Poepp.
Geófito rizomatoso. Endémica de Chile y Argentina (VII-XIV). Forma parte del sotobosque de Nothofagus pumilio, sobre $900 \mathrm{~m}$ de altitud. Exs. S. Teillier, J. Delaunoy \& C. Bonnemaison 6617, 6618 (CONC).

Caltha appendiculata Pers. maillico Geófito rizomatoso. Endémica de Chile y Argentina (VI-XII). Crece sólo en praderas húmedas de altura; escasa. Exs. S. Teillier, A. Marticorena, J. Macaya \& C. Bonnemaison 7393 (CONC).

Caltha sagittata Cav. maillico Geófito rizomatoso. Endémica de Chile y Argentina (IV-XII). Crece sólo en las praderas húmedas de altura; poco frecuente. Exs. S. Teillier, J. Delaunoy \& C. Bonnemaison 6671 (CONC).

Ranunculus hydrophilus Gaudich.

Hemicriptófito repente. Endémica de Chile y Argentina (VIII, XI-XII); primera cita para la región de Los Ríos. Crece en sitios inundables en la laguna Trarolafquén. No se coleccionó.

Ranunculus minutiflorus Bertero ex Phil.

Hemicriptófito repente. Endémica de Chile y Argentina (IVXII): Se encontró en los claros húmedos de casi todos los tipos de bosque. Exs. S. Teillier, J. Delaunoy \& C. Bonnemaison 6870 , 6871, 6872, 7167 (CONC)

Ranunculus peduncularis Sm.

Hemicriptófito escapífero. Endémica de Chile y Argentina (IVXII). Crece en los humedales de altura y en el matorral subandino. Exs. S. Teillier, J. Macaya, J. Delaunoy \& C. Bonnemaison 6877, 7240 (CONC).

Ranunculus repens $\mathrm{L}$.

botón de oro Hemicriptófito repente. Alóctona asilvestrada (IV-XII), originaria de Eurasia. Es la especie más frecuente y abundante del género, crece en toda clase de sitios húmedos; especialmente en los que estuvieron o están sujetos a perturbaciones humanas. Exs. S. Teillier, J. Delaunoy \& C. Bonnemaison 6875, 6876, 7168 (CONC).

Ranunculus spegazzinii Lourteig

Hemicriptófito repente. Endémica de Chile y Argentina (IXXII). Se la encontró en un pequeño curso de agua en la laguna Trarolafquén, donde crece con los tallos sumergidos, pero las hojas y las flores emergen sobre la superficie del agua. Exs. S. Teillier, J. Delaunoy \& C. Bonnemaison 6878 (CONC).

\section{Ranunculus trichophyllus Chaix}

Hidrófito. Especie nativa no endémica (V-XII). Crece como planta sumergida en la laguna Trarolafquén. No se coleccionó.

RHAMnACEAE

Colletia hystrix Clos chacay, crucero Nanofanerófito. Endémica de Chile y Argentina (III-XII). Se la encontró sólo en el valle del Pillanleufú, poco frecuente. Exs. S. Teillier, J. Macaya, J. Delaunoy \& C. Bonnemaison 7285, 7548 (CONC).

Discaria chacaye (G.Don) Tortosa chacay Nanofanerófito o caméfito en espaldera. Endémica de Chile y 
Argentina (V-XII). Crece en el bosque de Nothofagus antarctica en sitios húmedos; también forma parte del matorral subandino donde crece muy achaparrada. Exs. S. Teillier, J. Delaunoy \& C. Bonnemaison 6646, 6647, 6648, 6649, 6650 (CONC).

\section{RosACEAE}

Acaena antarctica Hook. f.

Hemicriptófito en roseta. Endémica de Chile y Argentina (XIVXII). Crece en el bosque de Nothofagus pumilio y en el matorral subandino, en sitios abiertos y húmedos. Exs. S. Teillier, J. Delaunoy \& C. Bonnemaison 6640, 6642 (CONC); S. Teillier, J. Macaya, A. Marticorena \& C. Bonnemaison 7412, 7442 (CONC).

\section{Acaena leptacantha Phil.}

Hemicriptófito en roseta. Endémica de Chile y Argentina (RM-X). Crece en sitios abiertos del matorral subandino. Exs. S. Teillier, J. Delaunoy \& C. Bonnemaison 6637, 6638, 6639 (CONC).

\section{Acaena magellanica (Lam.) Vahl}

Hemicriptófito en roseta. Especie nativa, no endémica, se encuentra en todas las regiones del país. Crece en el bosque de Nothofagus pumilio y en el matorral subandino, en sitios abiertos y húmedos. Exs. S. Teillier, J. Delaunoy \& C. Bonnemaison 6636, 7529 (CONC).

\section{Acaena ovalifolia Ruiz \& Pav.}

Hemicriptófito en roseta. Nativa, no endémica (IV, VI-XII) y luego entre VI-XII). Crece en los claros del bosque de tipo valdiviano y de Nothofagus dombeyi, también en las praderas degradadas; la más frecuente y abundante del género en el área. Exs. S. Teillier, J. Delaunoy \& C. Bonnemaison 6634, 6635, 6636-A, 7203 (CONC).

\section{Acaena pinnatifida Ruiz \& Pav.}

pimpinela Hemicriptófito en roseta. Endémica de Chile y Argentina (IV-XII). Crece en los claros y en los taludes del bosque de Nothofagus antarctica, en la pampa Pilmaiquén. Exs. S. Teillier, J. Delaunoy \& C. Bonnemaison 6632, 6633 (CONC).

\section{Fragaria chiloensis (L.) Mill.}

frutilla, lahueñe Hemicriptófito en roseta (estolonífero). Especie nativa, no endémica (VI-XI). Crece en los pastizales de los sitios abiertos, siendo particularmente abundante en la pampa Pilmaiquén. Exs. S. Teillier, J. Delaunoy \& C. Bonnemaison 6709 (CONC); S. Teillier, J. Macaya, J. Delaunoy \& C. Bonnemaison 7254 (CONC).

\section{Potentilla anserina L.}

hierba de la plata Hemicriptófito en roseta (estolonífero). Alóctona asilvestrada (IX-XIV), con origen en Eurasia. Se encontró sólo en la pampa Pilmaiquén, en la laguna Trarolafquén. Exs. S. Teillier, J. Delaunoy \& C. Bonnemaison 6876 (CONC); S. Teillier, J. Macaya, J. Delaunoy \& C. Bonnemaison 7224 (CONC).

\section{Rosa rubiginosa $\mathrm{L}$.}

rosa mosqueta Nanofanerófito. Alóctona asilvestrada (V-XI). Crece en sitios abiertos y alterados, hasta los $800 \mathrm{~m}$ de altitud. Exs. S. Teillier, J. Macaya, J. Delaunoy \& C. Bonnemaison 7244 (CONC).

Rubus constrictus P.J.Müll. \& Lefèvre

zarzamora Nanofanerófito. Alóctona asilvestrada (IX-X), de origen europeo. Se encuentra en sitios alterados, como praderas y márgenes de los bosques; hasta unos $900 \mathrm{~m}$ de altitud. Exs. S. Teillier, J. Macaya, J. Delaunoy \& C. Bonnemaison 7347 (CONC).

Rubus geoides $\mathrm{Sm}$. miñe-miñe Hemicriptófito en roseta (estolonífero). Endémica de Chile y Argentina (VIII-XI). Crece como sotobosque de Nothofagus pumilio y en el matorral subandino, escasa. Exs. S. Teillier, J. Delaunoy \& C. Bonnemaison 6863 (CONC); S. Teillier, J. Macaya, J. Delaunoy \& C. Bonnemaison 7239 (CONC).

\section{Rubus radicans Cav.}

Hemicriptófito en roseta (estolonífero). Endémica de Chile y Argentina (IX-XII). Crece en sitios húmedos en el bosque de tipo valdiviano y en el de Nothofagus antarctica, escasa. Exs. S. Teillier, J. Macaya, J. Delaunoy \& C. Bonnemaison 6862 (CONC); S. Teillier, J. Macaya, A. Marticorena \& C. Bonnemaison 7340 (CONC).

\section{RUBIACEAE}

Galium fuegianum Hook. f.

Hemicriptófito trepador. Endémica de Chile y Argentina (VI-XII). Se encontró en el bosque de Nothofagus antarctica y en el matorral subandino. Exs. S. Teillier, J. Delaunoy \& C. Bonnemaison 6901, 6902 (CONC)

Galium hypocarpium (L.) Endl. ex Griseb.

relbún Hemicriptófito trepador. Especie nativa, no endémica (II-XI). Se encontró en los claros y en los márgenes de los bosques de tipo valdiviano, de Nothofagus dombeyi y de Nothofagus antarctica. Exs. S. Teillier, J. Delaunoy \& C. Bonnemaison 6898, 6899, 6900, 7187 (CONC).

Galium magellanicum Hook. f.

Hemicriptófito escapífero. Endémica de Chile y Argentina (VIIIXII). Crece en sitios húmedos como la laguna Trarolafquén. Exs. S. Teillier, J. Delaunoy \& C. Bonnemaison 6896, 6897 (CONC).

Leptostigma arnottianum Walp.

Hemicriptófito repente. Endémica de Chile y Argentina (VIIIIX). Crece en las praderas húmedas. Exs. S. Teillier, J. Macaya, J. Delaunoy \& C. Bonnemaison 6752 (CONC).

Nertera granadensis (Mutis ex L. f.) Druce rucachucao Hemicriptófito repente. Especie nativa no endémica (VIII-XII). Forma parte del bosque de tipo valdiviano y del de Nothofagus dombeyi. Exs. S. Teillier, J. Macaya, J. Delaunoy \& C. Bonnemaison 6773, 7317 (CONC); S. Teillier, J. Delaunoy \& C. Bonnemaison 7165 (CONC).

Oldenlandia salzmannii (DC.) Benth. \& Hook.

Hemicriptófito repente. Especie nativa, no endémica (VII-X). Forma parte de la vegetación higrófila de la laguna Trarolafquén. Exs. S. Teillier, J. Macaya, A. Marticorena \& C. Bonnemaison 7428 (CONC).

SALICACEAE

Azara lanceolata Hook. f. aromo Megafanerófito. Endémica de Chile y Argentina (VIII-XI). Se la encuentra en los bosques de tipo valdiviano y de Nothofagus dombeyi. Exs. S. Teillier, J. Delaunoy \& C. Bonnemaison 6610, 7195 (CONC) 
Azara microphylla Hook. f.

chinchín Megafanerófito. Endémica de Chile y Argentina (IV, VII-X). Se la encuentra en los bosques de Nothofagus dombeyi. Exs. S. Teillier, J. Delaunoy \& C. Bonnemaison 6609 (CONC).

Salix fragilis L. sauce

Megafanerófito. Especie alóctona de origen europeo, está presente como árbol cultivado hace muchos años en Chile, pero no ha sido reportada como silvestre. Crece en el río Fuy. Exs. S. Teillier, J. Delaunoy \& C. Bonnemaison 7425 (CONC).

\section{SANTALACEAE}

Myoschilos oblongum Ruiz \& Pav.

orocoi

Nanofanerófito. Endémica de Chile y Argentina (IV-XII). Se la encuentra en los bosques de Nothofagus dombeyi y Nothofagus pumilio (lenga). Exs. S. Teillier, J. Delaunoy \& C. Bonnemaison 6786 (CONC).

\section{SAXIFRAGACEAE}

\section{Chrysosplenium valdivicum Hook.}

Hemicriptófito repente. Endémica de Chile y Argentina (IX-X). Crece en sitios muy húmedos del bosque de Nothofagus dombeyi, poco frecuente. Exs. S. Teillier, J. Delaunoy \& C. Bonnemaison 6667 (CONC).

\section{SCHOEPFIACEAE}

Quinchamalium chilense Molina

quimchamalí

Hemicriptófito escapífero. Nativa no endémica, presente en casi todo el país. Crece en el matorral subandino. Exs. S. Teillier, J. Macaya, A. Marticorena \& C. Bonnemaison 7411 (CONC).

\section{SCROPHULARIACEAE}

\section{Digitalis purpurea L.}

digital

Hemicriptófito en roseta. Alóctona asilvestrada (IX-X), de origen euroasiático. Crece en los claros del bosque de tipo valdiviano y de Nothofagus dombeyi. Exs. S. Teillier, J. Delaunoy \& C. Bonnemaison 6652, 6653 (CONC).

Euphrasia subexserta Benth.

Hemicriptófito escapífero. Endémica de Chile y Argentina (VI$\mathrm{X})$. Crece sólo en los humedales de altura del camino a Puerto Pirehueico, sobre $1.200 \mathrm{~m}$ de altitud. Exs. S. Teillier, J. Delaunoy \& C. Bonnemaison 6710, 6711 (CONC).

\section{Fonkia uliginosa Phil.}

Hemicriptófito repente. Género y especie endémicos de Chile y Argentina (IX-X). Es parte de la vegetación higrófila de la laguna Trarolafquén. Exs. S. Teillier, J. Delaunoy \& C. Bonnemaison 6686 (CONC); S. Teillier, J. Macaya, J. Delaunoy \& C. Bonnemaison 7238 (CONC); S. Teillier, J. Macaya, A. Marticorena \& C. Bonnemaison 7433 (CONC).

Kickxia elatine (L.) Dumort.

Terófito. Alóctona asilvestrada (RM-XIV), con origen en Eurasia. Se encontró en una pradera muy degradada. Exs. S. Teillier, J. Macaya, A. Marticorena \& C. Bonnemaison 7386 (CONC).

Mimulus luteus L.

berro amarillo Hemicriptófito en roseta. Endémica de Chile y Argentina. Crece en los esteros y en los ríos, a veces como planta flotante. Exs. S.
Teillier, J. Delaunoy \& C. Bonnemaison 6779 (CONC).

Ourisia alpina Poepp. \& Endl.

Hemicriptófito en roseta. Endémica de Chile y Argentina (VII-X). Se la observó sólo una vez en el matorral subandino de la parte más alta del camino a Puerto Pirehueico. Exs. S. Teillier, J. Delaunoy \& C. Bonnemaison 7544 (CONC).

Ourisia breviflora Benth. ssp. uniflora (Phil.) Meudt.

Hemicriptófito en roseta. Subespecie endémica de Chile y Argentina (VIII-X). Se la observó sólo una vez en un afloramiento de humedad en la parte más alta del camino a Puerto Pirehueico. Exs. S. Teillier, J. Delaunoy \& C. Bonnemaison 6834 (CONC).

Ourisia coccinea (Cav.) Pers.

Hemicriptófito en roseta. Endémica de Chile y Argentina (VIIIIX). Crece en sitios húmedos, incluso en los bordes de las quebradas y esteros o como sotobosque de Nothofagus dombeyi. Exs. S. Teillier, J. Delaunoy \& C. Bonnemaison 6832, 6833, 7166 (CONC).

Ourisia pygmaea Phil.

Hemicriptófito en roseta. Endémica de Chile y Argentina (VIII-X). Se la observó sólo una vez en la parte más alta del camino a Puerto Pirehueico, en un afloramiento de humedad. Exs. S. Teillier, J. Delaunoy \& C. Bonnemaison 6843-A (CONC).

Ourisia ruelloides (L. f.) Kuntze

Hemicriptófito en roseta. Endémica de Chile y Argentina (VI-XII). Crece en sitios tales como taludes y laderas con afloramientos de humedad y en las caídas de agua. Exs. S. Teillier, J. Delaunoy \& C. Bonnemaison 6831 (CONC); S. Teillier, J. Macaya, J. Delaunoy \& C. Bonnemaison 7241 (CONC).

Parentucellia viscosa (L.) Caruel

Terófito. Alóctona asilvestrada (RM-X), con origen en Europa. Crece en las praderas y es más frecuente en las de los sitios alterados. Exs. S. Teillier, J. Delaunoy \& C. Bonnemaison 7377 (CONC); S. Teillier, J. Macaya, A. Marticorena \& C. Bonnemaison 6828 (CONC)

Veronica peregrina L. ssp. xalapensis (Kunth) Pennell Terófito. Especie nativa, no endémica (IV-XII). Crece en las praderas húmedas, poco frecuente. Exs. S. Teillier, J. Delaunoy \& C. Bonnemaison 6916 (CONC).

Veronica serpyllifolia $\mathrm{L}$.

Hemicriptófito en roseta. Alóctona asilvestrada (VII-XIV). Crece en sitios húmedos alterados. Exs. S. Teillier, J. Delaunoy \& C. Bonnemaison 6914, 6915,7175 (CONC).

Solanaceae

Solanum crispum Ruiz \& Pav.

natri Nanofanerófito. Endémica de Chile y Argentina (IV-XI). Crece en el bosque de Nothofagus dombeyi; poco frecuente. Exs. S. Teillier, J. Delaunoy \& C. Bonnemaison 7169 (CONC).

Solanum furcatum Dunal hierba mora Hemicriptófito escapífero. Especie nativa no endémica, presente en todo el país. Crece en el bosque de Nothofagus dombeyi, en sitios 
sombríos y algo alterados; escasa. Exs. S. Teillier, J. Delaunoy \& C. Bonnemaison 6875 (CONC).

\section{Solanum palustre Schltdl.}

Hemicriptófito escapífero. Endémica de Chile y Argentina (VIII-X). Crece en el bosque de Nothofagus dombeyi, en sitios húmedos. Exs. S. Teillier, J. Delaunoy \& C. Bonnemaison 7172, 7243 (CONC); S. Teillier, J. Macaya, J. Delaunoy \& C. Bonnemaison 6876 (CONC).

\section{Solanum valdiviense Dunal}

huevil Nanofanerófito. Endémica de Chile y Argentina (VIII-X). Frecuente en el bosque de Nothofagus antarctica. Exs. S. Teillier, J. Delaunoy \& C. Bonnemaison 6877, 7170, (CONC); S. Teillier, J. Macaya, J. Delaunoy \& C. Bonnemaison 7275 (CONC); S. Teillier, J. Macaya, A. Marticorena \& C. Bonnemaison 7171 (CONC).

Thymelaeaceae

Ovidia andina (Poepp. \& Endl.) Meisner traro-voqui Nanofanerófito. Endémica de Chile y Argentina (VII-XI). Crece en el bosque de Nothofagus antarctica y en el matorral subandino, sobre $1.000 \mathrm{~m}$ de altitud. Exs. S. Teillier, J. Delaunoy \& C. Bonnemaison 6829, 6830, 7159 (CONC).

\section{Urticaceae}

\section{Pilea elliptica Hook. f.}

Hemicriptófito repente. Endémica de Chile (IX-XI). Crece en el bosque de tipo valdiviano, en un sector muy húmedo y sombrío; se la observó sólo en el sendero del río Truful. Exs. S. Teillier, J. Delaunoy \& C. Bonnemaison 6823 (CONC).

\section{Urtica magellanica Poir.}

Hemicriptófito escapífero. Especie nativa, no endémica (IV-XII). Crece en el bosque de tipo valdiviano y en el de Nothofagus dombeyi; en sitios húmedos y sombríos. Exs. S. Teillier, J. Delaunoy \& C. Bonnemaison 7173 (CONC).

\section{VALERIANACEAE}

\section{Valeriana lapathifolia Vahl}

hualhuilque

Hemicriptófito en roseta. Endémica de Chile y Argentina (IXXII). Crece en sitios algo sombríos en los bosques de Nothofagus dombeyi y de N. pumilio, y en el matorral subandino. Exs. S. Teillier, J. Delaunoy \& C. Bonnemaison 6917 (CONC).

\section{Valeriana polemoniifolia Phil.}

Terófito. Endémica de Chile y Argentina (VII-X). Se encontró en los claros del bosque de Nothofagus dombeyi. Exs. S. Teillier, J. Delaunoy \& C. Bonnemaison 6872, 6873, 6874, 6875 (CONC).

\section{Valeriana virescens $\mathrm{Clos}$}

Terófito. Endémica de Chile y Argentina (VII-XI). Crece tanto en el bosque de tipo valdiviano, como en el de Nothofagus dombeyi. Exs. S. Teillier, J. Delaunoy \& C. Bonnemaison 6870, 6871 (CONC); S. Teillier, J. Macaya, A. Marticorena \& C. Bonnemaison 7375 (CONC).

\section{VERBENACEAE}

Rhaphithamnus spinosus (Juss.) Moldenke arrayán macho Megafanerófito o nanofanerófito. Endémica de Chile y Argentina (IV-XI). Crece en el bosque de tipo valdiviano y en el de Nothofagus dombeyi. Exs. S. Teillier, J. Delaunoy \& C. Bonnemaison 6869 (CONC).

\section{ViOLACEAE}

Viola reichei Skottsb.

Hemicriptófito en roseta. Endémica de Chile y Argentina (VIIXII). Tiene una amplia distribución geográfica y ecológica en el área, se encuentra casi todos los tipos de comunidades vegetales, salvo la altoandina. Exs. S. Teillier, J. Delaunoy \& C. Bonnemaison 6910, 7445 (CONC); S. Teillier, J. Macaya, J. Delaunoy \& C. Bonnemaison 7230, 7322 (CONC); S. Teillier, J. Macaya, A. Marticorena \& C. Bonnemaison 7174 (CONC).

VITACEAE

Cissus striata Ruiz \& Pav. voqui-colorado Fanerófito trepador. Especie endémica de Chile y Argentina (IVXII). Crece de preferencia en el bosque de tipo valdiviano. Exs. S. Teillier, J. Delaunoy \& C. Bonnemaison 6666 (CONC).

WINTERACEAE

Drimys andina (Reiche) R.Rodr. \& Quezada canelo enano Nanofanerófito. Especie endémica de Chile (VIII-X). Crece en los bosques de Nothofagus pumilio, a veces, en los de N. dombeyi $\mathrm{o}$ en el matorral subandino. Exs. S. Teillier, J. Delaunoy \& C. Bonnemaison 6644, 6645 (CONC).

\section{MonocotyledonAtAe (LILIOPSIDA)}

Alstroemeriaceae

Alstroemeria aurea Graham liuto amarillo Geófito con raíces tuberosas. Endémica de Chile y Argentina (VII$\mathrm{XI})$. Crece en los claros de los bosques y a lo largo de los caminos; desde la parte baja hasta el bosque de Nothofagus pumilio. Exs. S. Teillier, J. Delaunoy \& C. Bonnemaison 6620 (CONC).

\section{BRomeliaceAe}

Fascicularia bicolor (Ruiz \& Pav.) Mez subsp. canaliculata E.C.Nelson \& Zizka chupalla Hemicriptófito en roseta y epífito. Endémica de Chile (V-X). Crece en el bosque de tipo valdiviano; poco frecuente. Hoffmann \& Flores (1989) la clasificaron como "vulnerable" por pérdida de hábitat. Exs. S. Teillier, J. Macaya, A. Marticorena \& C. Bonnemaison 7415 (CONC).

Greigia pearcei $\mathrm{Mez}$

ñocha Hemicriptófito en roseta. Endémica de Chile (VIII-X). Crece en el bosque de tipo valdiviano y en los de Nothofagus dombeyi (coigue) ubicados a menor altitud, poco frecuente. En el Libro Rojo de la Flora de Chile se clasificó como "insuficientemente conocida" (Hoffmann y Flores, 1989), sin embargo, los especialistas de la familia (Zizka et al. 2009) han propuesto su cambio a "en peligro"; la razón principal es la pérdida de hábitat. Exs. S. Teillier, J. Macaya, J. Delaunoy \& C. Bonnemaison 7309 (CONC).

\section{CORSIACEAE}

Arachnitis uniflora Phil. flor de la araña Hemicriptófito en roseta, saprófito. Especie nativa, no endémica (RM-XII). Se encuentra en el bosque de tipo valdiviano, donde es escasísima, se la observó en suelo con hojarasca de Persea lingue 
(lingue). No coleccionada.

\section{CYPERACEAE}

\section{Carex aematorrhyncha E.Desv.}

Hemicriptófito cespitoso. Endémica de Chile y Argentina (VIIIXII). Crece en sitios húmedos y arenosos. Exs. S. Teillier, J. Delaunoy \& C. Bonnemaison 7036, 7226 (CONC), 7037, 7206 (CONC, MIN); S. Teillier, J. Macaya, J. Delaunoy \& C. Bonnemaison 7226-A (CONC, MIN).

\section{Carex aphylla Kunth}

Hemicriptófito cespitoso. Endémica de Chile y Argentina (VI-X). Crece en sitios arenosos en los claros del bosque de Nothofagus antarctica y en el matorral subandino. Exs. S. Teillier, J. Delaunoy \& C. Bonnemaison 7039, 7040 (CONC).

\section{Carex banksii Boott}

Hemicriptófito cespitoso. Endémica de Chile y Argentina (V-XII). Crece en los humedales sobre los $1.000 \mathrm{~m}$ de altitud. Exs. S. Teillier, J. Delaunoy \& C. Bonnemaison 7041, 7042, 7535 (CONC), 7043 (CONC, MIN); S. Teillier, J. Macaya, J. Delaunoy \& C. Bonnemaison 7458, 7467 (CONC, MIN).

\section{Carex caduca Boott}

Hemicriptófito cespitoso. Endémica de Chile y Argentina (XIVXII). Crece en humedales de altura donde es escasa; a unos 1.000 $\mathrm{m}$ de altitud. Exs. S. Teillier, J. Delaunoy \& C. Bonnemaison 7044 (CONC, MIN).

\section{Carex canescens L.}

Hemicriptófito cespitoso. Especie nativa no endémica (XIV-XII) Crece en los pastizales y humedales entre 500 y $1.500 \mathrm{~m}$ de altitud. Exs. S. Teillier, J. Delaunoy \& C. Bonnemaison 7045, 7047, 7048, 7049 (CONC), 7046, 7050 (CONC, MIN); S. Teillier, J. Macaya, J. Delaunoy \& C. Bonnemaison 7460 (CONC, MIN).

\section{Carex decidua Boott}

Hemicriptófito cespitoso. Endémica de Chile y Argentina (RMXII). Crece en los pastizales húmedos sobre $1.000 \mathrm{~m}$ de altitud, poco frecuente. Exs. S. Teillier, J. Delaunoy \& C. Bonnemaison 7054, 7062 (CONC, MIN); S. Teillier, J. Macaya, J. Delaunoy \& C. Bonnemaison 7468, 7469 (CONC, MIN).

Carex excelsa Poepp. ex Kunth cortadera Hemicriptófito cespitoso. Endémica de Chile y Argentina (IV$\mathrm{XI})$. Crece sólo en un humedal formado por un meandro del río Pillanleufú, en el sector El Vado. Exs. S. Teillier, J. Delaunoy \& C. Bonnemaison 7055 (CONC, MIN); S. Teillier, J. Macaya, J. Delaunoy \& C. Bonnemaison 7462 (CONC, MIN).

\section{Carex fuscula d'Urv.}

Hemicriptófito cespitoso. Especie nativa, no endémica (VIII-XII). Crece en las praderas donde existe algo más de humedad. Exs. S. Teillier, J. Delaunoy \& C. Bonnemaison 7051, 7053 (CONC), 7052 (CONC, MIN); S. Teillier, J. Macaya, J. Delaunoy \& C. Bonnemaison 7227,7233 (CONC), 7461 (CONC, MIN).

Carex macloviana d'Urv.

Hemicriptófito cespitoso. Especie nativa, no endémica (VIIIXII). Crece en las praderas húmedas, una de las especies de Carex más frecuente en humedales. Exs. S. Teillier, J. Delaunoy \& C. Bonnemaison 7058, 7059 (CONC), 7057, 7060, 7061 (CONC, MIN); S. Teillier, J. Macaya, J. Delaunoy \& C. Bonnemaison 7459, 7463, 7464 (CONC, MIN).

\section{Cyperus reflexus Vahl} morcacho Hemicriptófito cespitoso. Especie nativa; no endémica (V$\mathrm{X})$. Crece en sitios húmedos, algo arenosos. Exs. S. Teillier, J. Delaunoy \& C. Bonnemaison 7035 (CONC).

\section{Eleocharis macrostachya Britton}

Hemicriptófito cespitoso. Especie nativa no endémica (IV-XII). Crece en humedales, más frecuente en la orilla de pequeños cursos de agua. Exs. S. Teillier, J. Delaunoy \& C. Bonnemaison 7031, 7032 (CONC).

\section{Eleocharis melanostachys (D’Urv.) C.B.Clarke} Hemicriptófito cespitoso. Especie endémica de Chile y Argentina (III-XII). Crece en los humedales; de la pampa Pilmaiquén. Exs. S. Teillier, J. Delaunoy \& C. Bonnemaison 7033 (CONC).

\section{Eleocharis pachycarpa Desv.}

Hemicriptófito cespitoso. Especie nativa no endémica (IV-XII). Crece en la laguna Trarolafquén. Exs. S. Teillier, J. Delaunoy \& C. Bonnemaison 7028, 7029, 7034 (CONC).

Isolepis cernua (Vahl) Roem. \& Schult.

Hemicriptófito cespitoso. Especie nativa no endémica; en Chile crece en casi todas las regiones administrativas. Crece en sitios muy húmedos, especialmente a orillas de los esteros. Exs. S. Teillier, J. Delaunoy \& C. Bonnemaison 6746, 6747 (CONC); S. Teillier, J. Macaya, J. Delaunoy \& C. Bonnemaison 7251 (CONC).

Oreobolus obtusangulus Gaudich.

Hemicriptófito cespitoso. Endémica de Chile y Argentina (VIIIXII). Crece sólo en las praderas de altura, sobre los $1.200 \mathrm{~m}$ de altitud, en el camino a Puerto Pirehueico, escaso. Exs. S. Teillier, J. Delaunoy \& C. Bonnemaison 7027 (CONC); S. Teillier, J. Macaya, A. Marticorena \& C. Bonnemaison 7389 (CONC).

Schoenoplectus californicus (C.A.Mey.) Soják var. tereticulmis (Steud.) Vegetti

totora Geófito rizomatoso. Variedad nativa no endémica (XIV-XII). Crece en humedales, se la encontró sólo en la pampa Pilmaiquén, en la laguna Trarolafquén. Exs. S. Teillier, J. Delaunoy \& C. Bonnemaison 7026 (CONC).

\section{Uncinia andina G.A.Wheeler}

Hemicriptófito cespitoso. Endémica de Chile y Argentina (VIIIXIV). Se encontró en el bosque de Nothofagus antárctica, sector laguna Trarolafquén, escasa. Exs. S. Teillier, J. Delaunoy \& C. Bonnemaison 7021 (CONC, MIN); S. Teillier, J. Delaunoy, J. Macaya \& C. Bonnemaison 7470 (MIN).

Uncinia erinacea (Cav.) Pers.

cortadera Hemicriptófito cespitoso. Endémica de Chile (VII-XII). Crece en el bosque de tipo valdiviano y en los de Nothofagus dombeyi, poco frecuente. Exs. S. Teillier, J. Delaunoy \& C. Bonnemaison 7020 (CONC); S. Teillier, J. Delaunoy, J. Macaya \& C. Bonnemaison $7471(\mathrm{MIN})$ 
Uncinia phleoides (Cav.) Pers.

quinquín

Hemicriptófito cespitoso. Nativa no endémica (IV-XI). Se la encontró en el bosque de tipo valdiviano y en los de Nothofagus dombeyi; es la especie más frecuente y de distribución más amplia del género en la reserva. Exs. S. Teillier, J. Delaunoy \& C. Bonnemaison 7023, 7299 (CONC), 7207 (CONC, MIN); S. Teillier, J. Macaya, J. Delaunoy \& C. Bonnemaison 7473 (CONC, MIN).

\section{Uncinia scabriuscula G.A.Wheeler}

Hemicriptófito cespitoso. Endémica de Chile y Argentina (IXXI). Crece en la parte alta del bosque de Nothofagus dombeyi y en los de Nothofagus pumilio, escasa. Exs. S. Teillier, J. Delaunoy \& C. Bonnemaison 7022, 7509 (CONC); S. Teillier, J. Macaya, J. Delaunoy \& C. Bonnemaison 7472 (CONC, MIN).

\section{Uncinia tenuis Poepp. ex Kunth}

Hemicriptófito cespitoso. Especie nativa no endémica (IX-XII). Se encuentra en los bosques de tipo valdiviano o de Nothofagus dombeyi, poco frecuente. Exs. S. Teillier, J. Delaunoy \& C. Bonnemaison 7025, 7413 (CONC), 7173 (CONC, MIN); S. Teillier, J. Macaya, J. Delaunoy \& C. Bonnemaison 7474 (CONC).

DiosCoreaceAe

Dioscorea brachybotrya Poepp.

Geófito trepador. Endémica de Chile y Argentina (VII-X). Crece en los bosques de tipo valdiviano o de Nothofagus dombeyi, poco frecuente. Exs. S. Teillier, J. Delaunoy \& C. Bonnemaison 6651 (CONC).

\section{IRIDACEAE}

Libertia tricocca Phil. tequel-tequel Geófito con raíces tuberosas. Endémica de Chile (VII-XIV). Crece en el bosque de tipo valdiviano, muy escasa; se la observó sólo en el río Truful. Si bien no figura en el Libro Rojo de la Flora de Chile, algunos especialistas (Ravenna et al. 1998) recomendaron su inclusión en la categoría "vulnerable" por la reducción de su hábitat. Exs. S. Teillier, J. Delaunoy \& C. Bonnemaison 6753 (CONC).

Olsynium junceum (E.Mey. ex C.Presl) Goldblatt ssp. depauperatum (Phil.) R.Rodr. \& Martic.

Geófito con raíces tuberosas. Subespecie endémica de Chile y Argentina (VIII-X). Su distribución está restringida a los bosques de Nothofagus pumilio y al matorral subandino del volcán MochoChoshuenco, poco frecuente. No coleccionada.

\section{Sisyrinchium nanum Phil.}

Geófito con raíces tuberosas. Endémica de Chile y Argentina (XIV-XII). Crece en sitios húmedos e inundables en la laguna Trarolafquén. Exs. S. Teillier, J. Delaunoy \& C. Bonnemaison 6839,6840 (CONC).

\section{Sisyrinchium pearcei Phil.}

Geófito con raíces tuberosas. Endémica de Chile y Argentina (IXXII). Crece en el matorral subandino, sobre los $1.200 \mathrm{msnm}$. Exs. S. Teillier, J. Delaunoy \& C. Bonnemaison 6841 (CONC).

JUNCACEAE

Juncus bufonius L.
Terófito. Nativa no endémica; se encuentra en casi todas las regiones administrativas. Crece en sitios anegados y algo alterados. Exs. S. Teillier, J. Delaunoy \& C. Bonnemaison 6995 (CONC).

\section{Juncus cyperoides Laharpe}

Hemicriptófito cespitoso. Nativa no endémica (IV-XII). Crece en sitios anegados. Exs. S. Teillier, J. Delaunoy \& C. Bonnemaison 6999, 7000, 7504 (CONC). S. Teillier, J. Macaya, J. Delaunoy \& C. Bonnemaison 7451 (PRA).

\section{Juncus dichotomus Elliot}

Hemicriptófito cespitoso. Nativa no endémica (VII-X). Crece en sitios anegados. Exs. S. Teillier, J. Delaunoy \& C. Bonnemaison 6995-B, 6997 (CONC).

\section{Juncus aff. diemii Barros}

Hemicriptófito cespitoso. Endémica de Chile y Argentina (VIII-X). Crece en sitios húmedos. Exs. S. Teillier, J. Delaunoy, J. Macaya \& C. Bonnemaison 7455 (CONC).

\section{Juncus effusus L.}

Hemicriptófito cespitoso. Nativa no endémica (VII-XI). Crece en las vegas donde es la especie dominante. Exs. S. Teillier, J. Delaunoy \& C. Bonnemaison 7002, 7006 (CONC, PRA), 7003, 7006-A, 7008 (CONC); S. Teillier, J. Delaunoy, J. Macaya \& C. Bonnemaison 7452 (CONC, PRA).

\section{Juncus imbricatus Laharpe}

Hemicriptófito cespitoso. Nativa no endémica (IV-X). Crece en sitios húmedos. Exs. S. Teillier, J. Delaunoy \& C. Bonnemaison 6996, 7004-B (CONC); S. Teillier, J. Delaunoy, J. Macaya \& C. Bonnemaison 7453 (CONC, PRA).

\section{Juncus pallescens Lam.}

Hemicriptófito cespitoso. Nativa no endémica (III-XI). Crece en sitios inundados. Exs. S. Teillier, J. Delaunoy \& C. Bonnemaison 7005 (PRA); S. Teillier, J. Delaunoy, J. Macaya \& C. Bonnemaison 7454 (CONC).

\section{Juncus stipulatus Nees \& Meyen}

Hemicriptófito cespitoso. Nativa no endémica, se encuentra en todas las regiones administrativas del país. Crece en sitios húmedos. Exs. S. Teillier, J. Delaunoy \& C. Bonnemaison 7007A, 7007-B (CONC); S. Teillier, J. Macaya, A. Marticorena \& C. Bonnemaison 7376 (PRA), 7405 (CONC).

Juncus tenuis Willd.

Hemicriptófito cespitoso. Nativa no endémica (RM-XIV). Crece en sitios húmedos, alterados. Exs. S. Teillier, J. Delaunoy \& C. Bonnemaison 7004-A (CONC, PRA).

Luzula brachyphylla Phil.

Hemicriptófito cespitoso. Endémica de Chile y Argentina (X-XII). Crece en las praderas subandinas y andinas. Exs. S. Teillier, J. Delaunoy \& C. Bonnemaison 7013, 7014, 7016 (CONC), 7015 (CONC, PRA); S. Teillier, J. Delaunoy, J. Macaya \& C. Bonnemaison 7456 (CONC).

Luzula tristachya Desv.

Hemicriptófito cespitoso. Endémica de Chile (IV-X). Crece 
en las praderas intervenidas. Muy afín a L. campestris (L.) DC. (J. Kirschner com. pers.). Exs. S. Teillier, J. Delaunoy \& C. Bonnemaison 7009, 7011, 7017 (CONC), 7205 (CONC, PRA).

\section{Marsippospermum philippii (Buchenau) Hauman}

Hemicriptófito cespitoso. Endémica de Chile y Argentina (VIIIXII). Crece en las praderas húmedas de altura, en el sector más alto del camino a Puerto Pirehueico, a unos $1.450 \mathrm{~m}$ de altitud, escasa. S. Teillier, J. Delaunoy \& C. Bonnemaison 6803, 7536 (CONC).

\section{LUZURIAGACEAE}

Luzuriaga polyphylla (Hook.) J.E.Macbr.

quilineja Fanerófito trepador, poco leñoso. Endémica de Chile (VIIXII). Crece en los bosques de tipo valdiviano. Exs. S. Teillier, J. Delaunoy \& C. Bonnemaison 7421 (CONC); S. Teillier, J. Macaya, J. Delaunoy \& C. Bonnemaison 7308 (CONC).

Luzuriaga radicans Ruiz \& Pav. quilineja Fanerófito trepador, poco leñoso. Endémica de Chile y Argentina (VII-XI). Crece en el bosque de tipo valdiviano. Exs. S. Teillier, J. Macaya, J. Delaunoy \& C. Bonnemaison 7311, 7337 (CONC).

\section{ORCHIDACEAE}

\section{Chloraea gaudichaudii Brongn.}

Geófito con raíces tuberosas. Endémica de Chile y Argentina (IX-XII). Crece en los claros y en los taludes en el bosque de Nothofagus antarctica; se la observó sólo en la pampa Pilmaiquén. Exs. S. Teillier, D. Rusti \& C. Bonnemaison 7543 (CONC).

\section{Chloraea speciosa Poepp.}

Geófito con raíces tuberosas. Especie endémica de Chile y Argentina (VIII-X). Se encontró sólo en el camino a Puerto Pirehueico, en un humedal, a más de $1.400 \mathrm{~m}$ de altitud. Exs. S. Teillier, J. Macaya, J. Delaunoy \& C. Bonnemaison 7271 (CONC).

Codonorchis lessonii (Brongn.) Lindl.

palomita Geófito con raíces tuberosas. Especie endémica de Chile y Argentina (VII-XII). Crece desde el bosque de tipo valdiviano hasta el de Nothofagus pumilio. Exs. S. Teillier, J. Delaunoy \& C. Bonnemaison 7191 (CONC).

\section{Gavilea araucana (Phil.) M.N.Correa}

Geófito con raíces tuberosas. Endémica de Chile y Argentina (VIIXII). Crece en el bosque de tipo valdiviano; poco frecuente. Exs. S. Teillier, J. Delaunoy \& C. Bonnemaison 6696 (CONC).

\section{Gavilea odoratissima Poepp.}

Epifito. Endémica de Chile y Argentina (VII-XI). Crece en el bosque de tipo valdiviano, sólo sobre Nothofagus obliqua, muy escasa. No coleccionada.

\section{Philesiaceae}

Lapageria rosea Ruiz \& Pav.

copihue Fanerófito trepador, poco leñoso. Endémica de Chile (IV-X). Crece en el bosque de tipo valdiviano. Exs. S. Teillier, J. Delaunoy \& C. Bonnemaison 6749 (CONC).

Poaceae (Gramineae)

Agrostis capillaris L.

chépica

Hemicriptófito cespitoso. Alóctona asilvestrada (VII-XII), con origen en Eurasia. Crece en las praderas antropogénicas y en los márgenes y taludes de los caminos; abundante en los sitios alterados. Exs. S. Teillier, J. Delaunoy \& C. Bonnemaison 6941 (CONC); S. Teillier, J. Macaya, J. Delaunoy \& C. Bonnemaison 7490, 7491 (CONC).

Agrostis castellana Boiss. \& Reut.

Hemicriptófito cespitoso. Alóctona asilvestrada (RM-XII), con origen en Europa mediterránea. Crece en las praderas antropogénicas, y en los márgenes y taludes de los caminos, escasa. Exs. S. Teillier, J. Delaunoy \& C. Bonnemaison 6928, 6929 (CONC).

\section{Agrostis gigantea Roth}

Hemicriptófito cespitoso. Alóctona asilvestrada (IV-XII), con origen en Eurasia. Crece en las praderas antropogénicas, y en los márgenes y taludes de los caminos; donde es frecuente $\mathrm{y}$ abundante. Exs. S. Teillier, J. Delaunoy \& C. Bonnemaison 6930, 6931 (CONC).

\section{Agrostis insconspicua Kunze ex E.Desv.}

Hemicriptófito cespitoso. Endémica de Chile y Argentina (V-XII). Crece en los claros y en los márgenes y taludes de los caminos en el bosque de Nothofagus antarctica, poco frecuente. Exs. S. Teillier, J. Macaya, J. Delaunoy \& C. Bonnemaison 6932, 7492 (CONC); S. Teillier, A. Marticorena, J. Macaya \& C. Bonnemaison 7436 (CONC).

Agrostis leptotricha E.Desv. pasto de perdiz Hemicriptófito cespitoso. Endémica de Chile y Argentina (VIIXII). Crece en los claros y en los márgenes y taludes de los caminos, en el bosque de Nothofagus antarctica. Exs. S. Teillier, J. Delaunoy \& C. Bonnemaison 6933, 6934, 6935 (CONC); S. Teillier, J. Macaya, J. Delaunoy \& C. Bonnemaison 7493 (CONC).

\section{Agrostis philippiana Rúgolo \& De Paula}

Hemicriptófito cespitoso. Endémica de Chile y Argentina (VIIIXII). Crece en las praderas húmedas de altura, en la parte más alta del camino a Puerto Pirehueico y en el volcán Mocho-Choshuenco. Exs. S. Teillier, J. Delaunoy \& C. Bonnemaison 6937, 6938, 7532 (CONC).

Agrostis stolonifera L.

chépica

Hemicriptófito cespitoso. Alóctona asilvestrada (V-XII), con origen en Eurasia. Crece en las praderas antropogénicas y en las orillas de los caminos, donde es frecuente. Exs. S. Teillier, J. Delaunoy \& C. Bonnemaison 6939, 6940 (CONC); S. Teillier, J. Macaya, J. Delaunoy \& C. Bonnemaison 7494, 7495 (CONC).

\section{Aira caryophyllea L.}

Terófito. Alóctona asilvestrada (IV-XII), con origen en Eurasia. Crece en las praderas intervenidas y en las orillas de los caminos, escasa. Exs. S. Teillier, J. Delaunoy \& C. Bonnemaison 7208 (CONC); S. Teillier, A. Marticorena, J. Macaya \& C. Bonnemaison 7399 (CONC).

\section{Anthoxanthum juncifolium (Hack.) Veldkamp}

Hemicriptófito cespitoso. Endémica de Chile y Argentina (IXXIV-X). Crece en los claros formando praderas o como sotobosque de Nothofagus antarctica donde es dominante, también en el 
matorral subandino. Exs. S. Teillier, J. Delaunoy \& C. Bonnemaison 6943, 6944, 7209 (CONC).

Anthoxanthum odoratum L. pasto oloroso Hemicriptófito cespitoso. Alóctona asilvestrada (IX-XII), originaria de Europa. Forma parte de las praderas antropogénicas donde es una de las dominantes. Exs. S. Teillier, J. Delaunoy \& C. Bonnemaison 6945 (CONC).

Arrhenatherum elatior (L.) P.Beauv. ex J.Presl \& C.Presl pasto cebolla Hemicriptófito cespitoso. Alóctona asilvestrada, originaria de Europa (VIII-XII). Forma parte de las praderas antropogénicas, escasa. Exs. S. Teillier, J. Delaunoy \& C. Bonnemaison 6946 (CONC).

Briza minor L. tembladerilla Terófita. Especie alóctona asilvestrada, originaria de Europa (III-X). Forma parte de las praderas antropogénicas. Exs. S. Teillier, A. Marticorena, J. Macaya \& C. Bonnemaison 7400 (CONC).

Briza subaristata Lam.

Hemicriptófito cespitoso. Nativa no endémica (IV-X). Forma parte de las praderas antropogénicas, escasa. Exs. S. Teillier, J. Delaunoy \& C. Bonnemaison 6947 (CONC).

\section{Bromus araucanus Phil.}

Hemicriptófito cespitoso. Endémica de Chile y Argentina (IXXII). Crece en los claros del bosque de tipo valdiviano (salto de Huilo Huilo). Exs. S. Teillier, J. Delaunoy \& C. Bonnemaison 6948 (CONC).

Bromus catharticus Vahl var. elata (E.Desv.) Planchuelo lanco Hemicriptófito cespitoso. Variedad nativa; se distribuye en todas las regiones administrativas del país. Crece en los claros del bosque de tipo valdiviano, en las praderas antropogénicas y en los márgenes de los caminos. Exs. S. Teillier, J. Delaunoy \& C. Bonnemaison 6949 (CONC); S. Teillier, J. Delaunoy, J. Macaya, \& C. Bonnemaison 7249, 7496,7497 (CONC).

\section{Bromus lithobius Trin.}

Hemicriptófito cespitoso. Endémica de Chile y Argentina (IV-XII). Crece en los claros del bosque de tipo valdiviano y de Nothofagus antarctica. Exs. S. Teillier, J. Delaunoy \& C. Bonnemaison 6950 (CONC); S. Teillier, J. Delaunoy, J. Macaya, \& C. Bonnemaison 7323 (CONC).

\section{Chusquea culeou E.Desv.}

colihue Fanerófita, gramínea. Endémica de Chile y Argentina (VII$\mathrm{XI}$ ). Crece en todos los tipos de bosque, principalmente en los claros que resultan por la tala. Exs. S. Teillier, J. Delaunoy \& C. Bonnemaison 6953, 6954, 7210 (CONC); S. Teillier, J. Delaunoy, J. Macaya \& C. Bonnemaison 7228, 7341 (CONC).

Chusquea valdiviensis E.Desv. quila Fanerófita, gramínea. Endémica de Chile y Argentina (IX-XIV-X). Crece en el bosque de tipo valdiviano, escasa (río Truful). Exs. S. Teillier, J. Delaunoy \& C. Bonnemaison 6951, 6952 (CONC); S. Teillier, J. Delaunoy, J. Macaya, \& C. Bonnemaison 7325 (CONC).
Cortaderia araucana Stapf

cortadera Hemicriptófito cespitoso. Endémica de Chile y Argentina (VIIXII). Crece en sitios húmedos en toda la reserva. Exs. S. Teillier, J. Delaunoy \& C. Bonnemaison 6957 (CONC).

Cortaderia egmontiana (Roem. \& Schult.) M.Lyle ex Connor Hemicriptófito cespitoso. Especie endémica de Chile y Argentina (RM-XII). Crece en las praderas húmedas de altura. Exs. S. Teillier, J. Delaunoy \& C. Bonnemaison 6956 (CONC).

Cynosurus echinatus L.

cola de zorro Terófito. Alóctona asilvestrada (V-XII), originaria de Eurasia. Crece en las praderas antropogénicas y otros sitios alterados. Exs. S. Teillier, J. Delaunoy, J. Macaya, \& C. Bonnemaison 7291 (CONC).

Dactylis glomerata L.

pasto ovillo Terófito. Alóctona asilvestrada (V-XII), originaria de Eurasia. Crece en las praderas antropogénicas y en otros sitios alterados. Exs. S. Teillier, J. Delaunoy, J. Macaya \& C. Bonnemaison 7282 (CONC).

Danthonia chilensis E.Desv. var. glabriflora Nicora cepilla Hemicriptófito cespitoso. Variedad endémica de Chile y Argentina (XIV-X). Crece en las praderas húmedas, en la laguna Trarolafquén. Exs. S. Teillier, J. Delaunoy \& C. Bonnemaison 6959 (CONC).

Elymus angulatus J.Presl

Hemicriptófito cespitoso. Nativa no endémica (II-XII). Crece en los espacios abiertos de los matorrales subandinos y en el margen de los bosques de Nothofagus. Exs. S. Teillier, J. Delaunoy \& C. Bonnemaison 6960, 6961, 6962 (CONC).

Festuca purpurascens Banks \& Sol. ex Hook. f.

Hemicriptófito cespitoso. Endémica de Chile y Argentina (VIIIXII). Crece en los claros de los bosques y en los taludes de los caminos. Exs. S. Teillier, J. Delaunoy \& C. Bonnemaison 6964, 6965, 6966, 6967 (CONC).

Festuca thermarum Phil.

Hemicriptófito cespitoso. Endémica de Chile y Argentina (VIII-XII). Crece en las praderas de altura y como sotobosque de Nothofagus antarctica. Exs. S. Teillier, J. Delaunoy \& C. Bonnemaison 6963, 7211, 7531 (CONC); S. Teillier, J. Macaya, J. Delaunoy \& C. Bonnemaison 7498 (CONC).

Holcus lanatus L.

pasto miel Hemicriptófito cespitoso. Alóctona asilvestrada (IV-XII), con origen en Eurasia. Crece en las praderas antropogénicas y en los bordes de los caminos donde es la especie dominante. Exs. S. Teillier, J. Delaunoy \& C. Bonnemaison 6968 (CONC).

\section{Hordeum parodii Covas}

Hemicriptófito cespitoso. Endémica de Chile y Argentina, distribución en Chile, desconocida. Crece en una pradera húmeda en la laguna Trarolafquén. Exs. S. Teillier, J. Delaunoy \& C. Bonnemaison 6969 (CONC); S. Teillier, J. Macaya, J. Delaunoy \& C. Bonnemaison 7229 (CONC).

Leptophyllochloa micrathera (E.Desv.) C.E.Calderón ex Nicora 
Hemicriptófito cespitoso. Endémica de Chile y Argentina (VII-X). Crece en una pradera húmeda de la laguna Trarolafquén. Exs. S. Teillier, J. Delaunoy \& C. Bonnemaison 6752 (CONC); S. Teillier, J. Macaya, J. Delaunoy \& C. Bonnemaison 7499 (CONC).

Lolium multiflorum Lam.

ballica italiana Hemicriptófito cespitoso. Alóctona, proveniente de Europa mediterránea, asilvestrada en casi todo el país. Crece en las praderas antropogénicas. Exs. S. Teillier, J. Delaunoy \& C. Bonnemaison 6972 (CONC).

Paspalum dasypleurum Kunze ex E.Desv. maicillo Hemicriptófito cespitoso. Endémica de Chile y Argentina (RM$\mathrm{X})$. Crece en las praderas antropogénicas, la única gramínea nativa frecuente en ese tipo de ambiente. Exs. S. Teillier, J. Delaunoy \& C. Bonnemaison 6973, 6974 (CONC).

\section{Phleum alpinum L.}

Hemicriptófito cespitoso. Nativa no endémica (IV-XII). Crece en las praderas húmedas de altura; se la observó en el camino a Puerto Pirehueico, a unos $1.200 \mathrm{~m}$ de altitud. Exs. S. Teillier, J. Delaunoy \& C. Bonnemaison 6975, 6976 (CONC).

Poa annua L. piojillo Terófito. Alóctona asilvestrada, proveniente de Eurasia, se encuentra en casi todo el país. Crece en sitios húmedos y alterados. Poco frecuente. No coleccionada.

Poa obvallata Steud.

Hemicriptófito cespitoso. Nativa no endémica (VII-XII). Crece tanto en el bosque de Nothofagus antarctica como en el matorral subandino y en el herbazal altoandino. Exs. S. Teillier, J. Delaunoy \& C. Bonnemaison 6980, 6981, 6982 (CONC, US), 6983, 6984, 6985 (CONC); S. Teillier, J. Macaya, J. Delaunoy \& C. Bonnemaison 7245 (CONC, US), 7248 (CONC).

Poa pratensis L.

Hemicriptófito cespitoso. Alóctona asilvestrada, proveniente de Eurasia, se encuentra en casi todo el país. Crece en las praderas antropogénicas. Frecuente y localmente abundante. Exs. S. Teillier, J. Delaunoy \& C. Bonnemaison 6977 (CONC).
Poa stenantha Trin.

Hemicriptófito cespitoso. Nativa no endémica (VII-XII). Crece en las praderas húmedas, sobre $1.000 \mathrm{~m}$ de altitud. Exs. S. Teillier, J. Macaya, J. Delaunoy \& C. Bonnemaison 7380 (CONC).

Podagrostis sesquiflora (E.Desv.) Parodi ex Nicora Hemicriptófito cespitoso. Endémica de Chile y Argentina (VII-X). Crece en los claros del bosque valdiviano, muy escasa (salto de Huilo Huilo). Exs. S. Teillier, J. Delaunoy \& C. Bonnemaison 6821 (CONC).

Polypogon australis Brongn. cola de ratón Hemicriptófito cespitoso. Endémica de Chile y Argentina; en el país se distribuye en casi todas las regiones administrativas, excepto en Magallanes. Crece en sitios húmedos, lechos de ríos, esteros y otros humedales. Exs. S. Teillier, J. Delaunoy \& C. Bonnemaison 6987 (CONC).

Rytidosperma pictum (Nees \& Meyen) Nicora Hemicriptófito cespitoso. Endémica de Chile y Argentina (IVXII). Crece en las praderas de altura. Exs. S. Teillier, J. Delaunoy \& C. Bonnemaison 6466, 6988, 6989, 6990 (CONC); S. Teillier, J. Macaya, J. Delaunoy \& C. Bonnemaison 7250, 7501 (CONC).

Stipa poeppigiana Trin. \& Rupr.

Hemicriptófito cespitoso. Nativa no endémica (V-X). Crece en los claros del bosque de Nothofagus antarctica y de Nothofagus dombeyi. Exs. S. Teillier, J. Delaunoy \& C. Bonnemaison 6991 (CONC).

Trisetum spicatum (L.) K. Richt.

Hemicriptófito cespitoso. Endémica de Chile y Argentina (V-XII). Crece en las praderas húmedas de altura, camino a Puerto Pirehueico, sobre los $1.200 \mathrm{~m}$ de altitud. Exs. S. Teillier, J. Delaunoy \& C. Bonnemaison 6918, 6993, 7530 (CONC); S. Teillier, J. Macaya, J. Delaunoy \& C. Bonnemaison 7502 (CONC); S. Teillier, A. Marticorena, J. Macaya \& C. Bonnemaison, 7383 (CONC).

Vulpia bromoides (L.) Gray pasto sedilla Terófito. Alóctona asilvestrada (III-XII), originaria de Europa. Crece en las praderas antropogénicas, escasa. Exs. S. Teillier, J. Delaunoy \& C. Bonnemaison 7346 (CONC).

Recibido: 18.01 .13

Aceptado: 12.04 .13 